\title{
THE AUTOMORPHISM GROUP OF A SHIFT OF FINITE TYPE
}

\author{
MIKE BOYLE, DOUGLAS LIND AND DANIEL RUDOLPH
}

\begin{abstract}
Let $\left(X_{T}, \sigma_{T}\right)$ be a shift of finite type, and $G=\operatorname{aut}\left(\sigma_{T}\right)$ denote the group of homeomorphisms of $X_{T}$ commuting with $\sigma_{T}$. We investigate the algebraic properties of the countable group $G$ and the dynamics of its action on $X_{T}$ and associated spaces. Using "marker" constructions, we show $G$ contains many groups, such as the free group on two generators. However, $G$ is residually finite, so does not contain divisible groups or the infinite symmetric group. The doubly exponential growth rate of the number of automorphisms depending on $n$ coordinates leads to a new and nontrivial topological invariant of $\sigma_{T}$ whose exact value is not known. We prove that, modulo a few points of low period, $G$ acts transitively on the set of points with least $\sigma_{T}$-period $n$. Using $p$-adic analysis, we generalize to most finite type shifts a result of Boyle and Krieger that the gyration function of a full shift has infinite order. The action of $G$ on the dimension group of $\sigma_{T}$ is investigated. We show there are no proper infinite compact $G$-invariant sets. We give a complete characterization of the $G$-orbit closure of a continuous probability measure, and deduce that the only continuous $G$-invariant measure is that of maximal entropy. Examples, questions, and problems complement our analysis, and we conclude with a brief survey of some remaining open problems.
\end{abstract}

\section{TABLE OF CONTENTS}

$\begin{array}{llr}\S 1 . & \text { Introduction } & 71 \\ \S 2 . & \text { Markers and subgroups } & 74 \\ \S 3 . & \text { Residual finiteness and divisibility } \\ \S 4 . & \text { Nonisomorphic automorphism groups } & 82 \\ \S 5 . & \text { Symmetry } & 85 \\ \S 6 . & \text { Induced action on the dimension group } & 90 \\ \S 7 . & \text { Induced action on periodic points } & 95 \\ \S 8 . & \text { p-adic aspects of the gyration representation } & 99 \\ \S 9 . & \text { Compact invariant sets } & 102 \\ \S 10 . & \text { Orbits of measures } & 112\end{array}$

1. Introduction. Let $T$ be a square nonnegative integral matrix. Following Williams [Wi], we associate to $T$ a homeomorphism $\sigma_{T}$ of a totally disconnected compact space $X_{T}$ as follows. If $T$ is $r \times r$, form the directed graph with $r$ states or nodes, and with $T_{i j}$ symbols or edges from state $i$ to state $j$. Let $\mathcal{L}$ be the set of

Received by the editors February 23, 1987.

1980 Mathematics Subject Classification (1985 Revision). Primary 20B27, 54H20, 58F11, 58F20; Secondary 20E26, 34C $35,68 \mathrm{Q} 75$.

Key words and phrases. Shift of finite type, symbolic dynamics, automorphism group, dimension group, invariant measure, residual finiteness, gyration function, periodic points.

The authors were supported, respectively, by NSF Grants DMS-8601619, DMS-8320356, and DMS-8504701. 
symbols of this graph. Then $X_{T} \subset \mathcal{L}^{\mathbb{Z}}$ consists of those $x=\left(\ldots, x_{-1}, x_{0}, x_{1}, \ldots\right)$ with the terminal state of $x_{i-1}$ matching the initial state of $x_{i}$ for all $i \in \mathbb{Z}$. Points in $X_{T}$ may be thought of as infinite trips on the graph. Clearly $X_{T}$ is compact in the topology induced from the product topology on $\mathcal{L}^{\mathbb{Z}}$, and the shift $\sigma_{T}: X_{T} \rightarrow X_{T}$ defined by $\left(\sigma_{T} x\right)_{i}=x_{i+1}$ is a homeomorphism. This dynamical system $\left(X_{T}, \sigma_{T}\right)$ is called a shift of finite type or topological Markov shift. Such systems are intrinsically characterized as expansive homeomorphisms of totally disconnected compact spaces with canonical coordinates [Bo]. They play a prominent role not only in topological dynamics [DGS] and coding theory $[\mathbf{A C H}]$, but are also crucial to the analysis of hyperbolic diffeomorphisms [Sm]. We shall assume throughout that $\sigma_{T}$ is mixing, or, equivalently, that some power of $T$ is strictly positive. To avoid trivial exceptions, we also require $T \neq[1]$.

Let $G=\operatorname{aut}\left(\sigma_{T}\right)$ denote the group of homeomorphisms of $X_{T}$ commuting with $\sigma_{T}$. If $\varphi \in G$, then the fundamental observation of Curtis, Lyndon, and Hedlund $\left[\mathbf{H}\right.$, Theorem 3.4] shows there is an $n$ and a finite block map $f: \mathcal{L}^{2 n+1} \rightarrow \mathcal{L}$ so that $(\varphi x)_{i}=f\left(x_{i-n}, \ldots, x_{i+n}\right)$. It follows that $G$ is countable, and is discrete in the compact-open mapping topology. Despite the finite character of such mappings, very little is known about the algebraic structure of $G$. Hedlund $[\mathbf{H}]$ showed that for the full $k$-shift, aut $\left(\sigma_{[k]}\right)$ contains two involutions whose product has infinite order, and also a copy of every finite group. Ryan [Ry2] showed that the center of aut $\left(\sigma_{T}\right)$ contains only the group $\Sigma$ of powers of $\sigma_{T}$. However, it is still an open problem whether the automorphism group of the 2 -shift is generated by the shift and involutions in the group. Another example of our ignorance is the inability to settle the question whether the automorphism groups of the 2-shift and 3-shift are isomorphic.

Recently two new approaches to the structure of $G=\operatorname{aut}\left(\sigma_{T}\right)$ have been made. Boyle and Krieger [BK] used the action of $G$ on the invariant set of periodic points for $\sigma_{T}$ to construct a nontrivial homomorphism from $G$ to $\prod_{n=1}^{\infty} \mathbb{Z} / n \mathbb{Z}$ called the gyration function, and used this function to study $G$. Wagoner [Wa2], in analogy with $K$-theory, constructed a nontrivial representation of $G$ by using its action on the space of Markov partitions of $X_{T}$. Furthermore, he has shown [Wa1] that $G$ can be modelled by homeomorphisms commuting with a special diffeomorphism of the sphere $S^{q}$ for $q \geq 5$.

Our purpose is to study the algebraic properties of $G$ and the dynamics of its action on $X_{T}$ and some associated spaces. The general method used here to construct elements of $G$ goes back at least to Hedlund, and is usually called the "marker method." Roughly speaking, this method divides the symbols of a doubly infinite sequence into program and data, and the automorphism makes the program act on the data. The requirement of keeping program and data separated leads to certain complications. Special cases of this idea are used in $\S 2$ to show that $G$ contains the free group on countably many generators, as well as the direct sum of countably many copies of $\mathbb{Z}$ and of any countable collection of finite groups. However, $G$ does not contain a group with unsolvable word problem. Also, every subgroup of $G$ is residually finite, implying $G$ cannot contain a nontrivial divisible group, nor the infinite symmetric group. The finite type character of the shifts is related to the failure of divisibility in their automorphism groups, for in Example 3.9 we construct 
a subshift whose automorphism group contains $\mathbb{Q}$ with 1 corresponding to the subshift. Automorphisms constructed using markers have finite order, although their composition may not. Conversely, we show that any finite-order element in $G$ is obtained from a marker construction by using appropriate coordinates (Proposition 2.6).

Divisibility of elements of $G$ is discussed in $\S 3$. The main question, which remains open, is whether an infinite-order element can have $n$th roots for infinitely many $n$. An argument using Ryan's theorem on the center of $G$ shows that $\operatorname{aut}\left(\sigma_{[4]}\right)$ is not algebraically isomorphic to aut $\left(\sigma_{[2]}\right)$. However, we are not able to decide whether $\operatorname{aut}\left(\sigma_{[3]}\right)$ and $\operatorname{aut}\left(\sigma_{[2]}\right)$ are isomorphic. In $\S 4$ we present an example of two shifts of finite type with equal zeta-functions that have nonisomorphic automorphism groups.

The growth of the set $G_{n}\left(\sigma_{T}\right)$ of automorphisms depending on the central $n$ coordinates is doubly exponential in $n$. In $\S 5$ we define the symmetry of $\sigma_{T}$ to be

$$
s\left(\sigma_{T}\right)=\limsup _{n \rightarrow \infty} \frac{1}{n} \log \log \left|G_{n}\left(\sigma_{T}\right)\right|
$$

show that symmetry is a topological invariant, and prove that $\frac{1}{2} h\left(\sigma_{T}\right) \leq s\left(\sigma_{T}\right) \leq$ $h\left(\sigma_{T}\right)$, where $h\left(\sigma_{T}\right)$ is the topological entropy of $\sigma_{T}$. The precise value of $s\left(\sigma_{T}\right)$ is not known to us for any $T \neq[1]$.

Krieger has associated to $\sigma_{T}$ an automorphism $\widehat{T}$ of a countable ordered abelian group $\left(\mathcal{G}_{T}, \mathcal{G}_{T}^{+}\right)$called the dimension group. In $\S 6$ we outline this construction, and show that each $\varphi \in G$ induces an automorphism of $\left(\mathcal{G}_{T}, \mathcal{G}_{T}^{+}, \widehat{T}\right)$. The main question is whether this dimension group representation $\delta: \operatorname{aut}\left(\sigma_{T}\right) \rightarrow \operatorname{aut}(\widehat{T})$ is surjective. In Theorem 6.8 we prove that if the eigenvalues of $\widehat{T}$ are simple and no ratio of them is a root of unity, then for all sufficiently large $n$ the map $\delta: \operatorname{aut}\left(\sigma_{T}^{n}\right) \rightarrow \operatorname{aut}\left(\widehat{T}^{n}\right)$ is surjective. The argument uses the fact that if the eigenvalues of $T$ are simple, then $\operatorname{aut}\left(\widehat{T}^{n}\right)$ is finitely generated. The proof of this has the Dirichlet unit theorem as its priipal ingredient. Two examples complement the discussion, one of which shows that $\operatorname{aut}(\widehat{T})$ is not always finitely generated.

The period of a point is not altered under an automorphism, so $G$ acts on the set $Q_{n}$ of points with least $\sigma_{T}$-period $n$. Is this action transitive? In Theorem 7.2 we prove a strong form of transitivity on $Q_{n}$ for all sufficiently large $n$. However, we give an example with two fixed points which we show cannot be interchanged by any composition of finite-order automorphisms. Our analysis of the action of $G$ on periodic points implies certain algebraic properties of $G$, including that none of $G$, $G /[G, G]$, and $G / \Sigma$ are finitely generated. We conclude $\S 7$ with an example showing that the profinite topology on $G$ does not always coincide with that induced from the action of $G$ on periodic points.

Boyle and Krieger [BK] introduced the gyration function of $\varphi \in G$ to be the number $g\left(\varphi, \sigma_{T}\right)(n) \in \mathbb{Z} / n \mathbb{Z}$ indicating the total amount of twist given by $\varphi$ to the orbits of length $n$. The map $g: G \rightarrow \prod_{n=1}^{\infty} \mathbb{Z} / n \mathbb{Z}$ defined by

$$
g(\varphi)=\left(g\left(\varphi, \sigma_{T}\right)(1), g\left(\varphi, \sigma_{T}\right)(2), \ldots\right)
$$

is a homomorphism called the gyration representation. They prove that $g\left(\sigma_{[k]}, \sigma_{[k]}\right)$ has infinite order for $k \geq 2$. Using computer experimentation, we stumbled on the fact that $g\left(\sigma_{T}, \sigma_{T}\right)\left(p^{n}\right)$ converges $p$-adically, usually to a nonzero limit that turns 
out to be transcendental. This is the basis of our proof in $\S 8$ that if the product of the nonzero eigenvalues of $T$ is not \pm 1 , then $g\left(\sigma_{T}, \sigma_{T}\right)$ has infinite order. It is also a key ingredient in our proof that $\sigma_{T}$ is not a limit, in the periodic point topology on $G$, of products of finite-order elements (Proposition 8.3).

The search for nontrivial representations of $G$ leads naturally to studying $G$ invariant sets and measures on $X_{T}$. In Theorem 9.2 we prove that if a point is not $\sigma_{T}$-periodic, then its $G$-orbit is dense. A modification of this argument shows that if $Y \subset Z$ are $\sigma_{T}$-invariant compact subsets of $\sigma_{T}$, then $Z$ is in the $G$-orbit closure of $Y$ under the Hausdorff metric on compact subsets. In $\S 10$ we obtain a complete characterization of the $G$-orbit closure of a probability measure on $X_{T}$. Roughly speaking, a measure $\nu$ is in the $G$-orbit closure of $\mu$ when it has enough cumulative entropy to accommodate an approximate image of $\mu$. The precise formulation is given in Theorem 10.1. One simple consequence is that the measure of maximal entropy on $X_{T}$ is the only continuous $G$-invariant measure.

This work has benefitted substantially from numerous conversations with many people. We would like in particular to thank Ethan Coven, John Franks, Ralph Greenberg, Hang Kim, Bruce Kitchens, Neal Koblitz, Wolfgang Krieger, Gopal Prasad, Frank Rhodes, Jonathan Rosenberg, Fred Roush, John Smillie, and Jack Wagoner. We would also like to thank the Mathematical Science Research Institute, the IBM Thomas J. Watson Research Center, and the National Science Foundation for their support.

2. Markers and subgroups. We shall describe a method for building automorphisms of $G=\operatorname{aut}\left(\sigma_{T}\right)$, and use this method to construct subgroups of $\operatorname{aut}\left(\sigma_{T}\right)$ isomorphic to such groups as the direct sum of any countable collection of finite groups, the free group on infinitely many generators, and the direct sum of countably many copies of $\mathbb{Z}$. However, we show $G$ does not contain a group with unsolvable word problem, and in the next section that it does not contain a nontrivial divisible group. We next discuss some elaborations of this marker method used throughout this paper, and show that automorphisms of finite order coincide with those obtained from a marker construction by using an appropriate symbolic presentation of $X_{T}$.

Recall from $\S 1$ that the set of symbols $\mathcal{L}$ for $X_{T}$ is the collection of edges for the graph of $T$, and that points of $X_{T}$ are just allowed bi-infinite sequences of symbols. For $x \in X_{T}$ let $x[m, n]=x_{m} \cdots x_{n} \in \mathcal{L}^{n-m+1}$ be the block of coordinates of $x$ from $m$ to $n$. Let $B_{n}\left(X_{T}\right)$ be the set of allowed blocks of symbols of length $n$ for $T$. Put $B\left(X_{T}\right)=\bigcup_{n=1}^{\infty} B_{n}\left(X_{T}\right)$. Suppose $M \in B_{m}\left(X_{T}\right)$ and that $D \subset B_{k}\left(X_{T}\right)$ is a collection of blocks with $M D M=\{M D M: D \in D\} \subset B_{2 m+k}\left(X_{T}\right)$ such that for every $D \in D$ the block $M$ can overlap the concatenation $M D M$ in only the initial and final segments of length $m$ (this disallows even partial overlaps at the ends). Let $\pi$ be an arbitrary permutation of $D$. Define the action of a block map $\varphi_{\pi}$ on $x \in X_{T}$ as follows. For each $i$, if $x[i, i+2 m+k-1]=M D M$, define $\left(\varphi_{\pi} x\right)[i, i+2 m+k-1]=M \pi(D) M$. Require $\varphi_{\pi}$ to have no other action. Because the blocks from $M D M$ cannot overlap except for the marker $M$, this is a welldefined $\sigma$-invariant map of finite order, so $\varphi_{\pi} \in \operatorname{aut}\left(\sigma_{T}\right)$. The correspondence $\pi \leftrightarrow \varphi_{\pi}$ hence embeds the symmetric group sym $D$ of $D$ into aut $\left(\sigma_{T}\right)$. We shall show shortly that $|D|$ can be made arbitrarily large by an appropriate choice of $M$. This implies by Cayley's theorem that every finite group embeds into aut $\left(\sigma_{T}\right)$, and 
generalizes Hedlund's argument [H, Theorem 6.13] from the automorphism group of a full shift to that of a shift of finite type.

Blocks, or collections of blocks, with the kind of nonoverlapping property used above play a role for constructing continuous maps similar to that of Rohlin bases used to define measurable isomorphisms in ergodic theory [Sh, Chapter 10]. The idea has surfaced in several guises, such as prefix synchronization codes in information theory $[\mathbf{G}]$.

DEFINITION 2.1. Two blocks overlap if an initial segment of one coincides with a terminal segment of the other. A collection of blocks in $B\left(X_{T}\right)$ has only trivial overlaps if distinct blocks do not overlap and each block overlaps itself only in the entire block.

The following gives an ample supply of blocks with only trivial overlaps.

LEMMA 2.2. There is a collection $\mathcal{M}=\bigcup_{n=1}^{\infty} \mathcal{M}_{n} \subset B\left(X_{T}\right)$ such that $\mathcal{M}_{n}$ contains $n$ blocks of equal length, $\mathcal{M}$ has only trivial overlaps, and

$$
\mathcal{M M}=\left\{M M^{\prime}: M, M^{\prime} \in \mathcal{M}\right\} \subset B\left(X_{T}\right) .
$$

Proof. Since $\sigma_{T}$ is mixing, and $T \neq[1]$ by our convention, there must be a loop $i_{0} i_{1} \cdots i_{k} i_{0} \in B\left(X_{T}\right)$ of distinct symbols with $k \geq 1$. Furthermore, one of these symbols, which we can assume is $i_{0}$, is followed by a symbol $j_{1} \neq i_{1}$.

First suppose $j_{1} \neq i_{0}$. Choose a path of minimal length from $j_{1}$ to the loop, say $j_{1} j_{2} \cdots j_{r} i_{s}$. The case $r=0$ is possible and corresponds to $j_{1}=i_{s}$ for some $s \neq 0,1$. Define $A=i_{0} \cdots i_{k}, B=i_{0} j_{1} j_{2} \cdots j_{r} i_{s} \cdots i_{k} \in B\left(X_{T}\right)$. For $1 \leq q \leq n$ define $M_{n q}=A^{2} B^{q} A B^{n-q+1}$. Noting the positions of $i_{0}$ in these blocks, it follows from the above minimality of paths that $\mathcal{M}=\left\{M_{n q}: 1 \leq q \leq n, n \geq 1\right\}$ has only trivial overlaps, and that $\mathcal{M M} \subset B\left(X_{T}\right)$ by construction. Since the lengths $\left|M_{n q}\right|$ are equal for $1 \leq q \leq n$, the collections $\mathcal{M}_{n}=\left\{M_{n q}: 1 \leq q \leq n\right\}$ satisfy the conclusions.

The remaining possibility is for $j_{1}=i_{0}$. In this case let $A=i_{1} \cdots i_{q} i_{0}$, and put $M_{n q}=A^{2} i_{0}^{q} A i_{0}^{n-q+1}$. Again noting the positions of $i_{0}$ in the $M_{n q}$ shows that $\mathcal{M}_{n}=\left\{M_{n q}: 1 \leq q \leq n\right\}$ for $n \geq 1$ satisfy the conclusions.

We shall say that $G=\operatorname{aut}\left(\sigma_{T}\right)$ contains a group $H$ if there is an isomorphism of $H$ to a subgroup of $G$. Using the markers constructed in Lemma 2.2, we will show that $G$ contains several kinds of infinite groups. For clarity, the constructions are first carried out on convenient full shifts, then extended to general $\sigma_{T}$ by a substitution map.

THEOREM 2.3. The group aut $\left(\sigma_{T}\right)$ contains the direct sum of every countable collection of finite groups.

Proof. We first obtain the embedding when $\sigma_{T}$ is the full 3-shift on $\{0,1,2\}$, then extend to general $\sigma_{T}$ by a substitution map using markers from Lemma 2.2.

First suppose $X_{T}=\{0,1,2\}^{\mathbf{Z}}$, and let $D_{n}=\{0,1\}^{n}, M=2$. For $\pi \in \operatorname{sym}\left(D_{n}\right)$ define $\varphi_{\pi} \in G$ using blocks $M D_{n} M=2 D_{n} 2$ as above. This yields an embedding of $\operatorname{sym} D_{n}$ to a subgroup of $H_{n}$ of $G$. Since $\left(\varphi_{\pi} x\right)_{i}=2$ iff $x_{i}=2$ and blocks from $2 D_{n} 2$ can overlap those from $2 D_{m} 2$ only in the end symbols when $n \neq m$, elements of $H_{n}$ commute with those of $H_{m}$ for $n \neq m$. Thus $G$ contains $\bigoplus_{n=1}^{\infty} \operatorname{sym} D_{n}$, which clearly contains the direct sum of every countable collection of finite groups. 
For general $\sigma_{T}$, use Lemma 2.2 to find three markers $M_{0}, M_{1}$, and $M_{2}$ of equal length with only trivial overlaps, such that $M_{i} M_{j} \in B\left(X_{T}\right)$ for all $i, j$. If $\pi \in$ $\operatorname{sym} D_{n}=\operatorname{sym}\{0,1\}^{n}$, define $\varphi_{\pi}$ to replace a block of the form $M_{2} M_{i_{1}} \cdots M_{i_{n}} M_{2}$, where $i_{1} \cdots i_{n} \in D_{n}$, with $M_{2} M_{j_{1}} \cdots M_{j_{n}} M_{2}$, where $\pi\left(i_{1} \ldots i_{n}\right)=j_{1} \cdots j_{n}$, and have no other action. The nonoverlapping nature of the $M_{i}$ shows that $\varphi_{\pi}$ is well-defined, that $\pi \mapsto \varphi_{\pi}$ embeds $\operatorname{sym}\left(D_{n}\right)$ into aut $\left(\sigma_{T}\right)$, and that the embedded subgroups commute. The proof now concludes as in the first case.

THEOREM 2.4. The group aut $\left(\sigma_{T}\right)$ contains the free product of any finite number of 2-element groups. Thus it contains the free group on two generators, hence the free group on a countable number of generators.

PROOF. We embed the free product of three copies of $\mathbb{Z} / 2 \mathbb{Z}$, the generalization to more copies being routine. We first work on a special full shift, then carry this over to a general $\sigma_{T}$.

Let the alphabet be $\mathcal{L}=\{0,1,2,3, *\}$, and $\sigma_{\mathcal{L}}$ be the full shift on $\mathcal{L}$. Define involutions $\varphi_{j}$ for $j=1,2,3$ as follows. All will be 2 -block maps, and each will exchange three pairs of 2-blocks. Specifically, $\varphi_{j}$ exchanges $s 0$ with $s j$ for $s \in$ $\mathcal{L} \backslash\{0, j\}$. Thus each $\varphi_{j}$ uses three markers for its definition, and has the important property that markers defining its action are not affected by it. It follows that each $\varphi_{j} \in \operatorname{aut}\left(\sigma_{\mathcal{L}}\right)$. Let $P$ be the free product of the 2-element groups $\{e, j\}$ for $j=1,2,3$. Define a homomorphism from $P$ to aut $\left(\sigma_{\mathcal{L}}\right)$ by mapping a reduced word $w=j_{n} \cdots j_{1} \in P$ to $\psi=\varphi_{j_{n}} \cdots \varphi_{j_{1}}$. Since each $\varphi_{j}^{2}=I$, the identity, this is welldefined. Consider the point $x=\cdots 0000 * 0000 \cdots$, with $x_{0}=*$. Then $(\psi x)_{n}=j_{n}$, $\left(\varphi_{j_{n}}^{-1} \psi x\right)_{n-1}=j_{n-2}$ and so on. This means that inductively $\psi$ determines the spelling of $w$, so this mapping embeds $P$ into aut $\left(\sigma_{\mathcal{L}}\right)$. It is elementary group theory that $P$ contains the free group $F_{2}$ of two generators [MKS, §1.4], and it is known [Ro, Theorem 11.27] that the commutator subgroup of $F_{2}$ is the free group on a countable number of generators.

This idea generalizes to arbitrary $\sigma_{T}$ by using markers instead of symbols. If $\mathcal{L}_{T}$ is the alphabet for $\sigma_{T}$, for each $a \in\{0,1,2,3, *\}$ use Lemma 2.2 to construct a marker $M_{a}$ over $\mathcal{L}_{T}$, all of equal length with only trivial overlaps, and beginning with and followed by $i_{0}$. Define involutions $\varphi_{j}, 1 \leq j \leq 3$, to exchange $M_{s} M_{0} i_{0}$ with $M_{s} M_{j} i_{0}$ for $s \in \mathcal{L} \backslash\{0, j\}$, and have no other effect. Since these markers have only trivial overlaps, the $\varphi_{j}$ are well-defined. The argument that they generate the free product of three copies of $\mathbb{Z} / 2 \mathbb{Z}$ is exactly as before.

REMARK 2.5. This theorem shows that $G$ is not amenable.

THEOREM 2.6. The group aut $\left(\sigma_{T}\right)$ contains the countable direct sum of copies of $\mathbb{Z}$.

PROOF. We first perform the embedding when $\sigma_{T}$ is the full shift on the alphabet $\mathcal{L}=\{0,1, a, b, c\}$, then generalize. Let $M_{n}=a b^{n} c$. Then $\mathcal{M}=\left\{M_{n}: n \geq 1\right\}$ has only trivial overlaps. For each $n \geq 1$ we will define two involutions $\alpha_{n}, \beta_{n}$, and then put $\varphi_{n}=\alpha_{n} \beta_{n}$. The idea behind this construction is contained in [L2], where it is used to construct automorphisms with interesting entropies. Define $\alpha_{n}$ to switch $M_{n} i j M_{n}$ with $M_{n} j i M_{n}$, where $i, j \in\{0,1\}$, and to have no other action. Define $\beta_{n}$ to map $r i M_{n} j s$ to $j M_{n} i$, where $i, j, r, s \in\{0,1\}$, and $M_{n}$ does not move. Declare $\beta_{n}$ to have no other action. Clearly $\alpha_{n}$ and $\beta_{n}$ are well-defined involutions that do not 
move any $M_{k}$, and only affect those symbols 0 and 1 adjacent to the appearances of $M_{n}$ and that are not adjacent to markers with subscript distinct from $n$. If $\varphi_{n}=\alpha_{n} \beta_{n}$, it follows that the $\varphi_{n}$ commute, but have no other relations. The action of $\varphi_{n}$ on the point $\left(M_{n} 00\right)^{\infty}\left(M_{n} 01\right)\left(M_{n} 00\right)^{\infty}$ is to shift the block 01 to the left by $\left|M_{n}\right|+2$, proving that $\varphi_{n}$ has infinite order. In fact, each $\varphi_{n}$ has topological entropy $\log 4$ [L2]. Thus the subgroup of $G$ generated by the $\varphi_{n}$ is isomorphic to the countable direct sum of copies of $\mathbb{Z}$.

The generalization to arbitrary $\sigma_{T}$ using markers $M_{0}, M_{1}, M_{a}, M_{b}$, and $M_{c}$ should now be routine.

This method allows the embedding of many kinds of countable groups into $G$. But is there a reasonable answer to the following?

\section{PROBLEM 2.7. Characterize the subgroups of aut $\left(\sigma_{T}\right)$.}

At least two properties of countable groups prevent them from being embeddable into aut $\left(\sigma_{T}\right)$. One is the lack of residual finiteness, which we consider in the next section. For the other, recall that a finitely presented group is said to have solvable word problem if there is an algorithm to decide whether a word in the generators represents the identity. There are countable groups without this property [Ro, Chapter 12]. We are endebted to Bruce Kitchens for the following observation.

PROPOSITION 2.8. The group aut $\left(\sigma_{T}\right)$ contains no finitely generated group with unsolvable word problem.

ProOF. Suppose a subgroup $K$ of $G=\operatorname{aut}(\sigma)$ has $n$ generators. The inverse of an automorphism is explicitly computable, if only by trying all block maps using coordinates from $-k$ to $k$ and increasing $k$ until the inverse is found. Say that $\varphi \in G$ has range at most $m$ if $(\varphi x)_{i}$ depends on only $x_{i-m}, \ldots, x_{i+m}$. There is an $m$ so that all the generators and their inverses have range at most $m$. Then a word $\psi$ of length $r$ in the generators and their inverses has range at most $r m$. As a block map, $\psi$ is explicitly determined by the block maps inducing the generators and their inverses. To check whether $\psi=I$, it is only necessary to see if $\psi\left(x_{-r m}, \ldots, x_{r m}\right)=x_{0}$ for all allowed blocks of length $2 r m+1$, a finite procedure. Thus $K$ has solvable word problem.

That a finitely presented subgroup of aut $\left(\sigma_{T}\right)$ has solvable word problem follows from the residual finiteness of aut $\left(\sigma_{T}\right)$, considered in the next section. Proposition 2.8 is stronger. There exist finitely generated residually finite groups with unsolvable word problem. For these facts see Theorem 4.6 in Chapter 4 of [LS] and the remarks that follow.

In each of the constructions above we have used a version of the marker method. By this we mean that the automorphism permutes certain blocks when they occur in the context of certain finite marking patterns, so that the marking patterns are not altered by the permutation. A useful point of view is that these marking patterns act as "program" on the "data" of blocks to be permuted. Invariance of the marking patterns is a reflection of the necessary separation of program from data. For the automorphisms $\varphi_{\pi}$ constructed at the beginning of this section, the marking pattern is a pair of $M$ 's separated by $k$ symbols, while the data is the collection $D$ of blocks permuted by $\pi$. In more elaborate constructions the marking patterns can be quite complicated (see the proofs of Theorem 9.2 and Lemma 10.7), 
but the automorphisms produced will have finite order. At the other extreme, an empty set of marking patterns corresponds to an automorphism that permutes symbols. The following result, apparently first observed by John Franks, shows that the finite-order elements of aut $\left(\sigma_{T}\right)$ are precisely those that are obtained from a marker construction on a conjugate shift.

Proposition 2.9. Suppose $\varphi \in$ aut $\left(\sigma_{T}\right)$ has finite order. There is a shift of finite type $\sigma_{U}$ and a conjugacy $\psi: X_{T} \rightarrow X_{U}$ so that $\psi \varphi \psi^{-1}$ is a 1-block permutation of symbols in $X_{U}$.

PROOF. Let $P_{0}$ be the partition of $X_{T}$ into sets $\left\{x \in X_{T}: x_{0}=a\right\}$ for $a \in \mathcal{L}_{T}$. Suppose $\varphi^{k}=I$, and put $P=\bigvee_{j=0}^{k-1} \varphi^{-j} P_{0}$. Note that $\varphi$ permutes the atoms of $P$. Since $P$ has atoms that are compact and open, there is an $n \geq 1$ so that $\bigvee_{j=-n}^{n} \sigma^{-j} P_{0}$ refines $P$. Let $P_{1}=\bigvee_{j=-n}^{n} \sigma^{-j} P$. Then $P_{1}$ is a compact open partition of $X_{T}$ refining $P_{0}$, and a standard argument [B1] from symbolic dynamics shows that if $n$ is sufficiently large, $P_{1}$ is a 1-step Markov partition for $\sigma_{T}$ with transition matrix, say, $U$ indexed by the atoms of $P_{1}$. This gives a conjugacy $\psi: X_{T} \rightarrow X_{U}$. Since $\varphi$ commutes with $\sigma_{T}$, it will permute the atoms of $P_{1}$. Hence $\psi \varphi \psi^{-1}$ acts by permuting the symbols of $X_{U}$.

3. Residual finiteness and divisibility. In this section we will prove that $G=\operatorname{aut}\left(\sigma_{T}\right)$ is residually finite. Since this property is inherited by subgroups, it will follow that $G$ does not contain nontrivial divisible groups, nor the infinite symmetric group. We then discuss some divisibility properties of $G$. We conclude with a construction of a subshift whose automorphism group contains a copy of the rationals.

Recall [MKS, p. 116] that an abstract group $H$ with identity $I$ is called residually finite if the intersection of all its normal subgroups of finite index is $\{I\}$. This is equivalent to $H$ having enough homomorphisms to finite groups to separate points, and also to being able to embed $H$ into a product of finite groups. The profinite topology on $H$ is the coarsest making all homomorphisms from $H$ to finite groups continuous. Then $H$ is residually finite exactly when the profinite topology on $H$ is Hausdorff [MKS, Problem 2.4.24(a)]. Clearly a subgroup of a residually finite group is itself residually finite.

THEOREM 3.1. The group aut $\left(\sigma_{T}\right)$ is residually finite.

PROOF. Let $Q_{n}=Q_{n}\left(\sigma_{T}\right)$ denote the set of points in $X_{T}$ with least $\sigma_{T}$-period $n$. Since $\sigma_{T}$ is mixing, each $Q_{n}$ is finite. An automorphism $\varphi \in G=\operatorname{aut}\left(\sigma_{T}\right)$ is a topological conjugacy of $\sigma_{T}$ with itself, hence preserves $Q_{n}$. Thus for each $n \geq 1$, an automorphism $\varphi$ induces a permutation $\left.\varphi\right|_{Q_{n}}$ in the symmetric group $\operatorname{sym} Q_{n}$. Let $K_{n}$ denote the kernel of the map $\left.\varphi \mapsto \varphi\right|_{Q_{n}}$. The $K_{n}$ are normal in $G$, and since $\bigcup_{n=1}^{\infty} Q_{n}$ is dense in $X_{T}$, it follows that $\bigcap_{n=1}^{\infty} K_{n}=\{I\}$. Hence $G$ is residually finite.

A group $D$ is divisible if every element has roots of all orders [MKS, §6.2]. A consequence of residual finiteness is that complete divisibility cannot occur in $G$.

COROLlARY 3.2. The group aut $\left(\sigma_{T}\right)$ contains no nontrivial divisible groups.

Proof. Suppose $D$ is a nontrivial divisible subgroup. Then $D$ is residually finite by Theorem 3.1. Let $\varphi \neq I$ be in $D$, and $N$ be a normal subgroup of finite 
index in $D$ with $\varphi \notin N$. Put $n=|D / N|$. Suppose there were a $\psi$ in $D$ with $\psi^{n}=\varphi$. Then $N=(\psi N)^{n}=\psi^{n} N=\varphi N$, contradicting $\varphi \notin N$.

To point out the role that finite type plays in Corollary 3.2, we construct in Example 3.9 a subshift not of finite type whose automorphism group contains $\mathbb{Q}$. This construction can be amplified so the resulting automorphism group is exactly Q.

Denote by $S_{\infty}$ the group of permutations of the natural numbers fixing all but finitely many elements. J. Wagoner has raised the question of whether $G$ contains $S_{\infty}$. The following negative answer has also been found, independently, by Kim and Roush.

COROLLARY 3.3. The group aut $\left(\sigma_{T}\right)$ does not contain $S_{\infty}$.

PROOF. If $A_{\infty}$ is the infinite subgroup of $S_{\infty}$ consisting of the even permutations, then $A_{\infty}$ is the union of the finite simple alternating groups, so is also simple. If $S_{\infty}$ were contained in aut $\left(\sigma_{T}\right)$, then by Theorem 3.1 the subgroup $A_{\infty}$ would also be residually finite. But $A_{\infty}$ is infinite and simple, so is not residually finite.

Corollary 3.2 shows for example that $G$ does not contain $\mathbb{Q}$ or $\mathbb{Z}\left(p^{\infty}\right)=\mathbb{Z}[1 / p] / \mathbb{Z}$ for primes $p$. However, the proof does not rule out partial divisibility.

PROBLEM 3.4. Is $\mathbb{Z}[1 / p]$ contained in aut $\left(\sigma_{T}\right)$ for any prime $p$ ?

This amounts to asking whether there is an automorphism of infinite order with an infinite chain of $p$ th roots. Indeed, we are unable to decide the following.

PROBLEM 3.5. Is there an automorphism in aut $\left(\sigma_{T}\right)$ of infinite order having an $n$th root for infinitely many $n$ ?

Note that if $\varphi$ is such an automorphism, then it cannot be topologically conjugate to a mixing shift of finite type. For if $\varphi \cong \sigma_{U}$ and $\psi^{n}=\varphi$, then $\psi$ is also a mixing shift of finite type ([BK, Lemma 2.5] or [L1, Theorem 8]), hence $\psi$ is conjugate to some $\sigma_{V}$. Then the spectral radius $\lambda_{U}$ of $U$ would be a Perron number [L1, §1] with $n$th root $\lambda_{V}$, which is also a Perron number. But $\lambda_{U}$ has only finitely many nontrivial factorizations into Perron numbers [L1, Theorem 4], so it has only finitely many Perron roots.

Although we cannot characterize the subgroups of $\mathbb{Q}$ contained in $G$, there is a complete answer for subgroups of $\mathbb{Q} / \mathbb{Z}$.

PROPOSITION 3.6. A subgroup of $\mathbb{Q} / \mathbb{Z}$ is contained in aut $\left(\sigma_{T}\right)$ iff its $p$-torsion subgroup is finite for every prime $p$.

PROOF. Recall that $\mathbb{Q} / \mathbb{Z} \cong \bigoplus_{p} \mathbb{Z}\left(p^{\infty}\right)$ is the primary decomposition of $\mathbb{Q} / \mathbb{Z}$ [Ka, §3]. If $H \subset \mathbb{Q} / \mathbb{Z}$, then $H$ has primary decomposition $H \cong \bigoplus_{p} H_{p}$ with $H_{p} \subset \mathbf{Z}\left(p^{\infty}\right)$. If some $H_{p}$ is infinite, then it is $\mathbb{Z}\left(p^{\infty}\right)$, and then this divisible group would be contained in $G$, contradicting Proposition 3.1. If all the $H_{p}$ are finite, then $H$ is contained in $G$ by Theorem 2.3 .

We now turn to examining chains of roots of the identity $I$. If $\left\{n_{j}: j \geq 1\right\}$ is a sequence of integers $n_{j}>1$ for all $j$, call a prime $p$ good for the sequence if it divides at least one, but only finitely many, of the $n_{j}$. Call $p$ bad if it divides infinitely many of the $n_{j}$. Some primes may be neither good nor bad. We first discuss the case when the roots generate a finite subgroup of $G$. 
PROPOSITION 3.7. Let $\left\{n_{j}: j \geq 1\right\}$ be a sequence with $n_{j}>1$. Then there are $\varphi_{j} \in \operatorname{aut}\left(\sigma_{T}\right)$ with $\varphi_{0}=I, \varphi_{j}^{n_{j}}=\varphi_{j-1}$ for $j \geq 1$, and which generate a finite nontrivial subgroup of aut $\left(\sigma_{T}\right)$ iff $\left\{n_{j}\right\}$ has a good prime.

PROOF. First suppose there are $\varphi_{j}$ having the properties mentioned. By skipping to the first $\varphi_{j} \neq I$ and adjusting indices, we may assume $\varphi_{1} \neq I$. Let $p$ be a prime dividing the order $o\left(\varphi_{1}\right)$ of $\varphi_{1}$, and $\Phi$ denote the group generated by the $\varphi_{j}$. If $p$ were bad, then $\varphi_{1}$ would have a $p^{k}$ th root in $\Phi$ for every $k \geq 1$. This contradicts finiteness of $\Phi$. Since $p \mid o\left(\varphi_{1}\right)$ and $o\left(\varphi_{1}\right) \mid n_{1}$, it follows that $p$ is good.

Conversely, suppose $p$ is good. Choose the largest $j_{0}$ so that $p \mid n_{j_{0}}$. Let $\psi$ be a $p$ th root of $I$, say constructed using Theorem 2.3. Define $\varphi_{j}=I$ for $0 \leq j<j_{0}-1$, $\varphi_{j_{0}}=\psi$, and put $\varphi_{j}=\psi^{k}$ for $j>j_{0}$, where $k \equiv\left(n_{j_{0}+1} \cdots n_{j}\right)^{-1} \bmod p$. An easy calculation shows these $\varphi_{j}$ work.

Proposition 3.8. Suppose $\left\{n_{j}: j \geq 1\right\}$ is a sequence with $n_{j}>1$. Then there are $\varphi_{j} \in \operatorname{aut}\left(\sigma_{T}\right)$ with $\varphi_{0}=I, \varphi_{j}^{n_{j}}=\varphi_{j-1}$ for $j \geq 1$, and which generate an infinite subgroup of aut $\left(\sigma_{T}\right)$ iff $\left\{n_{j}\right\}$ has infinitely many good primes.

PROOF. First suppose there are $\varphi_{j}$ as described, and let $\Phi$ denote the subgroup of $G=\operatorname{aut}\left(\sigma_{T}\right)$ they generate. Since $\Phi$ is a union of cyclic groups, it is abelian. Suppose $p$ divides $o\left(\varphi_{j}\right)$. If $p$ were bad, then every element in $\Phi$ would have a $p$ th root. This would force $G$ to contain $\mathbb{Z}\left(p^{\infty}\right)$, contradicting Proposition 3.1. Thus every bad prime is relatively prime to every $o\left(\varphi_{j}\right)$. If $\left\{n_{j}\right\}$ had only finitely many good primes, then there is a $j_{0}$ so that for $j \geq j_{0}$ each $n_{j}$ is a product of bad primes. Thus $\left(o\left(n_{j}\right), n_{j}\right)=1$ for $j \geq j_{0}$, so $o\left(\varphi_{j+1}\right)=o\left(\varphi_{j}^{n_{j}}\right)=o\left(\varphi_{j}\right)$ for $j \geq j_{0}$, implying $\Phi$ is finite. This contradiction proves $\left\{n_{j}\right\}$ has infinitely many good primes.

Conversely, suppose $\left\{n_{j}\right\}$ has infinitely many good primes, say $p_{1}<p_{2}<\cdots$. Since each $p_{j}$ divides only finitely many $n_{k}$, by passing to a subsequence we can assume there are $n_{i_{1}}<n_{i_{2}}<\cdots$ such that $p_{j} \mid n_{i_{j}}$ for $j \geq 1$. Put $i_{0}=1$, and let $m_{j}=n_{i_{j-1}+1} \ldots n_{i_{j}}$. Clearly it suffices to find a chain of roots for the $m_{j}$ that generate an infinite subgroup.

Since $p_{j}$ is good, for each $j$ there is a $d_{j}$ so that $m_{1} \cdots m_{k} \not \equiv 0 \bmod p_{j}^{d_{j}}$ for all $k \geq 1$. Let $H_{j}=\mathbb{Z} / p_{j}^{d_{j}} \mathbb{Z}$, and put $H=\bigoplus_{j=1}^{\infty} H_{j}$. Let $a_{j, 0}=p_{j}^{d_{j}-1} \in H_{j}$. Inductively we can find $a_{j, k} \in H_{j}$ so that $m_{j+k} a_{j, k}=a_{j, k-1}$. Let $b_{j}=a_{1, j-1}+$ $a_{2, j-2}+\cdots+a_{j, 0} \in H$. Then the $b_{j}$ generate an infinite subgroup of $H$, and recalling that $p_{j} \mid m_{j}$ we find that $m_{j} b_{j}=b_{j-1}$. By Theorem 2.6, $H$ embeds into $G$. If $\varphi_{j}$ is the image of $b_{j}$ under this embedding, then the $\varphi_{j}$ satisfy the requirements.

In Corollary 3.2 we proved that the automorphism group of a shift of finite type cannot contain a divisible group such as $\mathbb{Q}$. If we drop the "finite type" hypothesis, divisible subgroups are possible. The following construction yields a minimal subshift $(X, \sigma)$ and an embedding of $\mathbb{Q}$ into aut $(X, \sigma)$.

The details of Example 3.9 are intricate, and the reader may wonder whether a more "natural" action of $\mathbb{Q}$ would suffice. Unfortunately, because $\mathbb{Q}$ is not locally compact, most natural actions of $\mathbb{Q}$ fail to be expansive, so cannot yield subshifts. For example, $\mathbb{Q}$ acts on $\{0,1\}^{\mathbb{Q}}$, but individual elements of this action are not expansive. Expansive maps with roots of arbitrary order are harder to come by.

EXAMPLE 3.9. A minimal subshift $(X, \sigma)$ and an embedding of $\mathbb{Q}$ into aut $(X, \sigma)$ so that $1 \in \mathbb{Q}$ corresponds to $\sigma \in \operatorname{aut}(X, \sigma)$. 
We first sketch how to construct a subshift $(X, \sigma)$ with an $n$th root. Suppose there are $n$ symbols $a_{0}, a_{1}, \ldots, a_{n-1}$, and one "spacer" symbol $s$. Further suppose that allowed blocks in $X$ have $a_{n-1}$ always preceded by an $s$, and $a_{0}$ always followed by an $s$. Define a block map $\varphi$ by $\varphi\left(a_{j}\right)=a_{j+1}$ for $0 \leq j \leq n-2$, and $\varphi\left(s a_{n-1}\right)=$ $a_{0} s$. In general, $\varphi(X)$ need not be $X$. However, if $X$ is designed so $\varphi(X)=X$, then $\varphi$ gives an automorphism of $(X, \sigma)$. As the iterates of $\varphi$ act on $x \in X$, different parts of $x$ are moved left one position at different iterates, much like the familiar slinky toy. The cumulative effect of $\varphi^{n}$ is to move every symbol to the left once, so $\varphi$ is an $n$th root of $\sigma$.

Before giving the detailed construction, let us describe the role of the objects and maps obtained. The construction will proceed by stages, starting with an initial alphabet $\mathcal{L}$. At stage $q \geq 3$ we will have $q$ ! words $w_{0}^{(q)}, \ldots, w_{q !-1}^{(q)}$ from $\mathcal{L}$ forming the set $\mathcal{W}^{(q)}$. Each $w_{j}^{(q)}$ will be a concatenation of words from $\mathcal{W}^{(q-1)}$ separated by 0,1 , or 2 spacer symbols $s$. The subshift $X$ will consist of all $x \in \mathcal{L}^{\mathbf{Z}}$ so that every subblock of $x$ is also a subblock of some constructed word. Every word from $W^{(q-1)}$ will occur in every word from $W^{(q)}$, and $s^{3}$ will never occur in any word. From this it will follow that every allowed block in $X$ occurs syndetically, so $(X, \sigma)$ will be minimal. It will also follow that if $x \in X$ and $q \geq 3$, then $x[-\infty, \infty]$ can be uniquely decomposed into a concatenation of words from $W^{(q)}$ separated by 0,1 , or 2 spacers $s$. For each $q \geq 3$ there will be maps $\varphi_{k}^{(q)}(3 \leq k \leq q)$ defined on words in $W^{(q)}$. They will have the properties that $\left(\varphi_{k}^{(q)}\right)^{k}=\varphi_{k-1}^{(q)}$ for $4 \leq k \leq q$, that $\left(\varphi_{3}^{(3)}\right)^{6}=\sigma$, and the consistency condition that $\varphi_{k}^{(q)}$ applied to a word in $W^{(q+r)}$ gives the same result as $\varphi_{k}^{(q+r)}$ would. Thus on $X$ the $\varphi_{k}^{(q)}(q \geq 3)$ consistently define a block map $\varphi_{k}$, and these obey $\varphi_{k}^{k}=\varphi_{k-1}(k \geq 4), \varphi_{3}^{6}=\sigma$. Hence mapping $1 / k$ ! to $\varphi_{k}$ for $k \geq 3$ embeds $\mathbb{Q}$ into aut $(X, \sigma)$, with 1 corresponding to $\sigma$.

To begin the construction, let the alphabet be $\mathcal{L}=\left\{a_{0}, a_{1}, \ldots, a_{5}, s\right\}$. For the initial stage $q=3$, put $w_{j}^{(3)}=a_{j}(0 \leq j \leq 5)$, and $\mathcal{W}^{(3)}=\left\{w_{j}^{(3)}: 0 \leq j \leq 5\right\}$. Define $\varphi_{3}^{(3)}\left(w_{j}^{(3)}\right)=w_{j+1}^{(3)}(0 \leq j \leq 4)$, and $\varphi_{3}^{(3)}\left(s w_{5}^{(3)}\right)=w_{0}^{(3)} s$. This is the method outlined in the first paragraph to obtain a 6th root of $\sigma$, so $\left(\varphi_{3}^{(3)}\right)^{6}=\sigma$, and we need only make sure that $\varphi_{3}^{(3)}(X)=X$.

Next we give the first inductive step, to $q=4$. Begin by defining for $0 \leq m \leq 3$ the 4 words

$$
w_{m}^{(4)}=w_{0}^{(3)}\left(s w_{0}^{(3)}\right)^{3}\left[\left(s w_{m}^{(3)}\right)\left(s w_{m+1 \bmod 6}^{(3)} \cdots\left(s w_{m+5 \bmod 6}^{(3)}\right)\right]\left(s w_{0}^{(3)}\right)^{4} .\right.
$$

We then obtain the 4 ! words in $\mathcal{W}^{(4)}$ by defining

$$
w_{4 r+m}^{(4)}=\left(\varphi_{3}^{(3)}\right)^{r}\left(w_{m}^{(4)}\right) \quad(0 \leq r \leq 5,0 \leq m \leq 3) .
$$

Note that every word in $\mathcal{W}^{(3)}$ occurs in every word in $\mathcal{W}^{(4)}$. Next put $\varphi_{4}^{(4)}\left(w_{j}^{(4)}\right)=$ $w_{j+1}^{(4)}(0 \leq j \leq 4 !-2)$, and $\varphi_{4}^{(4)}\left(s w_{4 !-1}^{(4)}\right)=w_{0}^{(4)} s$. Then let $\varphi_{3}^{(4)}=\left(\varphi_{4}^{(4)}\right)^{4}$. Words from $W^{(4)}$ are cyclically moved by $\varphi_{4}^{(4)}$ but break into 4 groups of 6 each on which $\varphi_{3}^{(4)}$ acts exactly as $\varphi_{3}^{(3)}$. Thus $\varphi_{3}^{(4)}$ is consistent with $\varphi_{3}^{(3)}$, and $\left(\varphi_{4}^{(4)}\right)^{4}=\varphi_{3}^{(4)}$ by construction. Since every word from $\mathcal{W}^{(3)}$ occurs in every word from $\mathcal{W}^{(4)}$, and $s^{3}$ never occurs here, the minimality conditions are satisfied at stage $q=4$. 
Suppose at stage $q-1$ we have defined $(q-1)$ ! words $w_{j}^{(q-1)}$ for $0 \leq j \leq$ $(q-1) !-1$ and maps $\varphi_{k}^{(q-1)}(3 \leq k \leq q-1)$ so that $\left(\varphi_{k}^{(q-1)}\right)^{k}=\varphi_{k-1}^{(q-1)}$ and the $\varphi_{k}^{(3)}, \ldots, \varphi_{k}^{(q-1)}$ are consistent. Construct stage $q$ as follows. First define, for $0 \leq j \leq q-1$,

$$
w_{m}^{(q)}=w_{0}^{(q-1)}\left(s w_{0}^{(q-1)}\right)^{q-1}\left[\prod_{i=0}^{(q-1) !-1} s w_{m+i \bmod (q-1) !}^{(q-1)}\right]\left(s w_{0}^{(q-1)}\right)^{q} .
$$

We then obtain the $q$ ! words in $\mathcal{W}^{(q)}$ by putting

$$
w_{q r+m}^{(q)}=\left(\varphi_{q-1}^{(q-1)}\right)^{r}\left(w_{m}^{(q)}\right) \quad(0 \leq r<(q-1) !, 0 \leq m \leq q-1) .
$$

Next define $\varphi_{q}^{(q)}$ by $\varphi_{q}^{(q)}\left(w_{j}^{(q)}\right)=w_{j+1}^{(q)}$ for $0 \leq j \leq q !-1$, and $\varphi_{q}^{(q)}\left(s w_{q !-1}^{(q)}\right)=w_{0}^{(q)} s$. Finally, set

$$
\varphi_{k}^{(q)}=\left(\varphi_{q}^{(q)}\right)^{q ! / k !} \quad(3 \leq k \leq q) .
$$

Then, as in the $q=4$ case, for fixed $k$ each $\varphi_{k}^{(p)}(k \leq p \leq q)$ is consistent with $\varphi_{k}^{(q)}$, and $\left(\varphi_{k}^{(q)}\right)^{k}=\varphi_{k-1}^{(q)}$. Every word in $\mathcal{W}^{(q-1)}$ occurs in every word of $\mathcal{W}^{(q)}$, and $s^{3}$ never occurs. Furthermore, because of the repetitions of $s w_{0}^{(q-1)}$ at the ends of the $w_{m}^{(q)}$, every $x \in X$ has $x[-\infty, \infty]$ decomposed uniquely as a concatenation of words from $W^{(q)}$ separated by no more than $2 s$ 's. This completes the construction of stage $q$.

The $\mathcal{W}^{(q)}$ and $\varphi_{k}^{(q)}$ constructed obey the conditions described in the second paragraph, and we thus obtain the required minimal subshift $(X, \sigma)$ and embedding of $\mathbb{Q}$ into aut $(X, \sigma)$.

REMARK 3.10. In our construction of $w_{j}^{(q)}$, the words from $\mathcal{W}^{(q-1)}$ are cyclically listed once. However, any arrangement of words from $\mathcal{W}^{(q-1)}$, with arbitrary repetitions, would also work, provided each word is used at least once. By using a long and highly recurrent listing of words from $\mathcal{W}^{(q-1)}$, it is possible to construct a uniquely ergodic subshift $(X, \sigma)$ so that $\operatorname{aut}(X, \sigma) \cong \mathbb{Q}$, and such that every Borel measurable mapping $\varphi: X \rightarrow X$ commuting with $\sigma$ is continuous. This should be contrasted with the uniquely ergodic Morse minimal subshift, whose automorphism group is just $\mathbb{Z} \oplus(\mathbb{Z} / 2 \mathbb{Z})$ with generators the shift and coordinate complementation (see $[\mathbf{C K}, \mathbf{C}]$ ).

4. Nonisomorphic automorphism groups. Conjugate subshifts of finite type have isomorphic automorphism groups. Also, clearly $\operatorname{aut}\left(\sigma_{T}\right)=\operatorname{aut}\left(\sigma_{T}^{-1}\right)$. Since there are shifts of finite type not conjugate to their inverse [PT, Proposition $3.30]$, there are nonconjugate shifts of finite type with isomorphic automorphism groups. However, the following question remains open.

QUESTION 4.1. If $\sigma_{U}$ is not conjugate to $\sigma_{T}$ or $\sigma_{T}^{-1}$, can $\operatorname{aut}\left(\sigma_{U}\right)$ and $\operatorname{aut}\left(\sigma_{T}\right)$ be isomorphic as abstract groups?

In this regard, we know of only one useful invariant, namely the theorem of Ryan [Ry 1, Ry2] that the center of $\operatorname{aut}\left(\sigma_{T}\right)$ is precisely the set of powers of $\sigma_{T}$. Thus, for example, as an abstract group $\operatorname{aut}\left(\sigma_{[4]}\right)=\operatorname{aut}\left(\sigma_{[2]}^{2}\right)$ has center generated by an element with a square root in the group, while aut $\left(\sigma_{[2]}\right)$ does not since the 
2-shift has no square root. The following example, a more refined application of this idea, shows that the isomorphism class of aut $\left(\sigma_{T}\right)$ is not determined by the zeta-function. The motivation for the specific matrices used lies in a consequence of [B2] that there is only one shift equivalence class over $\mathbb{Z}$ for matrices whose nonzero spectrum is $\{1,2\}$, while there are exactly two such classes for $\{1,8\}$, only one of which can correspond to the cube of the first class.

EXAMPLE 4.2. Two mixing shifts of finite type with equal $\zeta$-function and having nonisomorphic automorphism groups.

Let

$$
V=\left[\begin{array}{llll}
1 & 0 & 0 & 1 \\
0 & 1 & 0 & 1 \\
0 & 1 & 1 & 0 \\
1 & 0 & 1 & 0
\end{array}\right], \quad T=V^{3}, \quad U=\left[\begin{array}{ll}
7 & 6 \\
1 & 2
\end{array}\right] .
$$

Then $\varsigma_{T}(t)=\varsigma_{U}(t)=[(1-8 t)(1-t)]^{-1}$. Clearly the generator $\sigma_{T}$ for the center of aut $\left(\sigma_{T}\right)$ has a cube root $\sigma_{V}$. Suppose $\sigma_{U}$ has a cube root $\varphi$ in aut $\left(\sigma_{U}\right)$. Then $\varphi$ is again a shift of finite type ([BK, Lemma 2.5] or [L1, Theorem 8]), say $\varphi=$ $\sigma_{W}$. Since $\sigma_{U}=\left(\sigma_{W}\right)^{3} \cong \sigma_{W^{3}}$, the nonzero spectrum of $W$ is $\{1,2\}$ counting multiplicity. Hence by [B2], there is an integer $j$ so that $W$ is shift equivalent over $\mathbb{Z}$ to $\left[\begin{array}{ll}2 & 0 \\ j & 1\end{array}\right]=W_{1}$. Since

$$
W_{1}=\left[\begin{array}{ll}
1 & 0 \\
j & 1
\end{array}\right]\left[\begin{array}{cc}
2 & 0 \\
-j & 1
\end{array}\right], \quad\left[\begin{array}{cc}
2 & 0 \\
-j & 1
\end{array}\right]\left[\begin{array}{ll}
1 & 0 \\
j & 1
\end{array}\right]=\left[\begin{array}{ll}
2 & 0 \\
0 & 1
\end{array}\right]
$$

and

$$
U=\left[\begin{array}{cc}
1 & -1 \\
0 & 1
\end{array}\right]\left[\begin{array}{ll}
8 & 8 \\
1 & 2
\end{array}\right], \quad\left[\begin{array}{cc}
8 & 8 \\
1 & 2
\end{array}\right]\left[\begin{array}{cc}
1 & -1 \\
0 & 1
\end{array}\right]=\left[\begin{array}{ll}
8 & 0 \\
0 & 1
\end{array}\right],
$$

we would have

$$
W_{1}^{3}=N=\left[\begin{array}{ll}
8 & 0 \\
0 & 1
\end{array}\right] \text { and } M=\left[\begin{array}{ll}
8 & 0 \\
1 & 1
\end{array}\right]
$$

shift equivalent over $\mathbb{Z}$. Thus there would be matrices $R$ and $S$ over $\mathbb{Z}$ and a positive integer $l$ with $R S=M^{l}, S R=N^{l}, N R=R M$, and $S N=M S$. Now $N R=R M$ forces $R_{12}=0$ and $7 R_{21}=-R_{22}$. Also, $S N=M S$ forces $S_{12}=0$. Then $R S=M^{l}$ implies $R_{22} S_{22}=1$, so $R_{22}= \pm 1$. This contradicts $7 R_{21}=-R_{22}$.

5. Symmetry. Let $\sigma_{T}$ be a mixing shift of finite type. As in the proof of Proposition 2.8, say that $\varphi \in G=\operatorname{aut}\left(\sigma_{T}\right)$ has range at most $n$ if $(\varphi x)_{i}$ depends only on $x_{i-n}, \ldots, x_{i+n}$. A natural measure of the symmetry of $\sigma_{T}$ is the rate of growth of the subset $G_{n}\left(\sigma_{T}\right)$ of those automorphisms in $G$ with range at most $[n / 2]$. This growth turns out to be doubly exponential, so we define the symmetry of $\sigma_{T}$ as

$$
s\left(\sigma_{T}\right)=\limsup _{n \rightarrow \infty} \frac{1}{n} \log \log \left|G_{n}\left(\sigma_{T}\right)\right| .
$$

The definition of symmetry depends on a particular presentation of $\sigma_{T}$, but the following proves that symmetry is a conjugacy invariant. Suppose $\sigma_{T}$ and $\sigma_{U}$ are conjugate via a mapping $\psi: X_{T} \rightarrow X_{U}$. Choose $m$ so that both $\psi$ and $\psi^{-1}$ have range at most $m$. If $\varphi \in G_{n}\left(\sigma_{T}\right)$, then $\psi \varphi \psi^{-1} \in G_{n+4 m}\left(\sigma_{U}\right)$, so that $\left|G_{n+4 m}\left(\sigma_{U}\right)\right| \geq\left|G_{n}\left(\sigma_{T}\right)\right|$. Thus $s\left(\sigma_{U}\right) \geq s\left(\sigma_{T}\right)$, and by symmetry they coincide. 
THEOREM 5.1. If $\sigma$ is a mixing shift of finite type, then

$$
\frac{1}{2} h(\sigma) \leq s(\sigma) \leq h(\sigma) .
$$

PROOF. We first establish the upper bound. Represent $\sigma$ as $\sigma_{T}$ with $r$ states, and put $X=X_{T}$. Now $\left|G_{n}\left(\sigma_{T}\right)\right|$ is trivially bounded above by the total number of block maps $B_{n+1}(X) \rightarrow\{0, \ldots, r-1\}$, or $r^{\left|B_{n+1}(X)\right|}$. If $h\left(\sigma_{T}\right)=\log \lambda$, where $\lambda$ is the dominant eigenvalue for $T$, there is a $\kappa>0$ so that $\left|B_{n+1}(X)\right|<\kappa \lambda^{n}$. Thus $\left|G_{n}(\sigma)\right|<r^{\kappa \lambda^{n}}$, so that $s(\sigma) \leq \log \lambda=h(\sigma)$.

The lower bound uses the automorphisms constructed at the beginning of $\S 2$. For this we first need to show that the number of marker-free blocks of length $m$ between two markers grows faster than $\left(\lambda^{1-\varepsilon}\right)^{m}$, where $\varepsilon$ can be made small by choosing long enough markers.

Fix a small $\varepsilon>0$ and a marker $M \in B_{n}(X)$, where $n \geq n_{0}$ with $n_{0}$ to be determined. Let $l$ be a transition length for $T$, so that $T^{l}>0$. There is an $a>0$ so that $\left|B_{k}(X)\right|>a \lambda^{k}$ for $k \geq 1$. If $k \leq n$, then $M$ has $n-k+1$ subblocks of length $\mathrm{k}$. Hence if $k$ is chosen so that $a \lambda^{k}>n-k+1$, there will be a block $C \in B_{k}(X)$ that does not appear in $M$. If $n_{0}$ is large enough, a choice of $k<\frac{\varepsilon}{2} n-l$ is possible.

Consider blocks $M D M \in B_{N}(X)$ of the form

$$
M E C B_{1} C B_{2} \cdots C B_{K} C F M \text {, }
$$

where $B_{j} \in B_{n-2 k}(X), N=(n-k) K+2 n+2 l+k$, and $E, F$ are fixed transition blocks in $B_{l}(X)$. Since $l$ is a transition length, every block of $B_{n-2 k-2 l}(X)$ can be the central part of each $B_{j}$. Hence the collection $D$ of blocks $D$ with the required form has cardinality

$$
|D| \geq\left(a \lambda^{n-2 k-2 l}\right)^{K} \geq\left(a \lambda^{(1-\varepsilon) n}\right)^{K} .
$$

Since $2|C|+\left|B_{j}\right|=n$, a subblock of $D \in D$ with length $n$ must contain $C$. Now $M$ does not contain $C$, and has only trivial self-overlap. Thus $M$ can only occur in $M D M$ as the initial or terminal segment. As at the beginning of $\S 2$, distinct permutations $\pi \in \operatorname{sym} D$ determine distinct automorphisms $\varphi_{\pi} \in$ aut $\left(\sigma_{T}\right)$ whose range is clearly at most $N$. Thus

$$
\left|G_{2 N+1}\left(\sigma_{T}\right)\right| \geq\left[\left(a \lambda^{(1-\varepsilon) n}\right)^{K}\right] ! .
$$

Now Stirling's formula implies that $\log m !>m(\log m-1)$, which applied to the above yields

$$
\begin{aligned}
s\left(\sigma_{T}\right) & \geq \limsup _{N \rightarrow \infty} \frac{1}{2 N+1} \log \log \left|G_{2 N+1}\right| \\
& \geq \limsup _{K \rightarrow \infty} \frac{1}{2(n-k) K+4(n+l)+2 k+1} \log \left(a \lambda^{(1-\varepsilon) n}\right)^{K} \\
& \geq \frac{1-\varepsilon}{2} \log \lambda=\frac{1-\varepsilon}{2} h\left(\sigma_{T}\right) .
\end{aligned}
$$

Since $\varepsilon>0$ was arbitrary, the lower estimate is proved.

We do not know the exact value of $s\left(\sigma_{T}\right)$ for any $T \neq[1]$, nor whether the definition's lim sup is actually a limit. 


\section{PROBLEM 5.2. Compute $s\left(\sigma_{T}\right)$.}

For full shifts $\sigma_{[r]}$ this problem can be viewed as a quantitative inquiry into the relative sparseness of aut $\left(\sigma_{[r]}\right)$ in the semigroup of all block maps from $X_{[r]}$ to itself. A topological measure of this sparseness has been given by Sears [Se]. We remark that for $\sigma_{[r]}$, if we replace "automorphism" by "surjective map" in the definition of symmetry, the value is easily shown to be $\log r=h\left(\sigma_{[r]}\right)$. For any block map involving $k$ symbols can be used to define a right permutive block map using $k+1$ symbols by adding modulo $r$ the value of the block map on the initial $k$ symbols to the last coordinate. Such right permutive maps are surjective [H, Theorem 6.6].

NOTE ADDED IN PROOF. H. Kim and F. Roush have shown that $s\left(\sigma_{T}\right)=h\left(\sigma_{T}\right)$ for all irreducible $T$. Their solution to Problem 5.2 uses a modification of the construction used to prove Theorem 5.1.

Let $\mu_{T}$ be the measure of maximal entropy for $\sigma_{T}$. Coven and Paul [CP] proved that $\mu_{T}$ is $\varphi$-invariant for every $\varphi \in G$. Since $G$ is not amenable (Remark 2.5), the sets $G_{n}$ may provide a replacement for averaging sets for the action of $G$ on $\left(X_{T}, \mu_{T}\right)$.

QUESTION 5.3. If $f \in C\left(X_{T}\right)$, does $\left|G_{n}\right|^{-1} \sum_{\varphi \in G_{n}} f(\varphi x)$ converge to

$$
\int_{X_{T}} f d \mu_{T}
$$

in any reasonable sense for most or all $x \in X_{T}$ ?

6. Induced action on the dimension group. For this section only, we drop our standing assumption that $T$ be aperiodic, and assume only that $T$ is nonnegative integral and not nilpotent. Building on the fundamental work of Williams [Wi] and Elliot [E], Krieger [Kr2] associated to each shift of finite type $\sigma_{T}$ an orderpreserving automorphism $\widehat{T}$ of an ordered group $\left(\mathcal{G}_{T}, \mathcal{G}_{T}^{+}\right)$called the dimension group of $\sigma_{T}$. The triple $\left(\mathcal{G}_{T}, \mathcal{G}_{T}^{+}, \widehat{T}\right)$ is a topological invariant since it can be defined using only topological notions. Two shifts of finite type are shift equivalent exactly when their dimension triples are isomorphic [Kr2].

We first review an algebraic description of the dimension triple, and indicate its relationship to underlying topological notions. Next we show how an automorphism $\varphi$ of $\sigma_{T}$ induces an automorphism $\delta(\varphi)$ of the dimension triple. The basic problem is to determine whether $\delta$ is surjective. We do not settle this. We show in Theorem 6.8 that if the nonzero eigenvalues of $T$ are simple, and if the ratio of distinct eigenvalues is not a root of unity, then for all sufficiently large $n$ the map $\delta: \operatorname{aut}\left(\sigma_{T}^{n}\right) \rightarrow \operatorname{aut}\left(\widehat{T}^{n}\right)$ is surjective. We also show that these hypotheses on the eigenvalues of $T$ imply $\operatorname{aut}(\widehat{T})=\operatorname{aut}\left(\widehat{T}^{n}\right)$ for all $n$. Example 6.7 shows that in general $\operatorname{aut}\left(\widehat{T}^{2}\right)$ can be larger than aut $(\widehat{T})$, though still finitely generated. Also, using an idea suggested to us by Gopal Prasad, in Example 6.9 we exhibit a $T$ for which $\operatorname{aut}(\widehat{T})$ is not finitely generated.

Suppose $T$ is an $r \times r$ nonnegative integral matrix. For the moment we drop our standing assumption that $T$ be aperiodic. It will be convenient to have matrices act on the right, and for vectors to be row vectors. Say that $v \in \mathbb{Q}^{r}$ is eventually integral (under $T$ ) if $v T^{n} \in \mathbb{Z}^{r}$ for large enough $n$. Call two eventually integral vectors $v$ and $w$ equivalent if $v T^{n}=w T^{n}$ for large enough $n$. The set $\mathcal{G}_{T}$ of equivalence 
classes $[v]$ of eventually integral vectors $v$ inherits an additive group structure from $\mathbb{Q}^{r}$. The positive cone $\mathcal{G}_{T}^{+}$is the set of $[v]$ for the $v T^{n} \geq 0$ eventually.

Let $R=T^{r} \mathbb{Q}^{r}$ be the eventual range of $T$. Each class in $\mathcal{G}_{T}$ has a unique representative in $R$, so it will sometimes be convenient to regard $\mathcal{G}_{T}$ as embedded in $R$. Define $\widehat{T}$ on $\mathcal{G}_{T}$ by $\widehat{T}([v])=[v T]$. By considering $\mathcal{G}_{T}$ as a subgroup of $R$, the extension of $\widehat{T}$ to $R$ is invertible, so $\widehat{T}$ defines an automorphism of $\mathcal{G}_{T}$. It is clear that $\widehat{T}$ is order-preserving. By the dimension triple of $\sigma_{T}$ we shall mean $\left(\mathcal{G}_{T}, \mathcal{G}_{T}^{+}, \widehat{T}\right)$.

We now sketch Krieger's topological construction of the dimension triple. For a detailed introduction to these ideas, see [BMT, Chapters 2, 5, 11].

By an $n$-ray we shall mean a set of the form

$$
x(-\infty, n]^{*}=\left\{y \in X_{T}: y_{j}=x_{j} \text { for }-\infty<j \leq n\right\},
$$

where $x \in X_{T}$. By an $n$-beam we mean a finite disjoint union of $n$-rays. Note that although the notion of $n$-ray is tied to the presentation $T$, that of $n$-beam is not, since an $n$-beam can be topologically described as a finite union of compact open subsets of unstable sets in $X_{T}$ in the natural inductive limit topology. If $C=\bigcup_{j} x^{j}(-\infty, n]^{*}$ is an $n$-beam, define a vector $v_{C, n} \in \mathbb{Z}^{r}$ whose $i$ th entry is the number of $n$-rays $x^{j}$ such that $x_{n}^{j}$ has terminal state $i$. If $C$ is an $n$-beam, then it is also an $m$-beam for $m \geq n$, and $v_{C, m}=v_{C, n} T^{m-n}$. Define beams $C$ and $D$ to be equivalent if for large enough $k$ we have $v_{C, k}=v_{D, k}$. The set of equivalence classes generate the positive cone $\mathcal{G}^{+}$of an ordered group $\mathcal{G}$ using the definition $[C]+[D]=[C \cup D]$ if $C \cap D=\varnothing$. Now $\sigma_{T}$ acts on beams, preserves equivalence, so induces an order-preserving automorphism $\widehat{\sigma}_{T}$ of $\mathcal{G}$. Since equivalence of beams corresponds to equivalence of eventually integral vectors, it is routine to verify that the map sending the class $[C]$ of an $n$-beam to $\widehat{T}^{-n}\left(\left[v_{C, n}\right]\right)$ is an isomorphism of $\left(\mathcal{G}, \mathcal{G}^{+}, \widehat{\sigma}_{T}\right)$ to the dimension triple $\left(\mathcal{G}_{T}, \mathcal{G}_{T}^{+}, \widehat{T}\right)$ defined above.

Therefore, to prove that the dimension triple is a topological invariant, it suffices to prove that if $\psi: \sigma_{T} \rightarrow \sigma_{U}$ is a topological conjugacy, then $\psi$ maps beams to beams, respects equivalence of beams, and intertwines $\widehat{\sigma}_{T}$ with $\widehat{\sigma}_{U}$. The routine verifications are omitted. However, we add one note of caution. If $\psi: X_{T} \rightarrow X_{U}$ is merely surjective, it is not necessarily true that $\psi$ maps beams to beams.

We shall denote the group of order-preserving automorphisms of the dimension triple by aut $(\widehat{T})$. Suppose $\varphi \in \operatorname{aut}\left(\sigma_{T}\right)$. Since $\varphi$ is a self-conjugacy of $\sigma_{T}$, the argument above about topological invariance proves that $\varphi$ induces an automorphism $\delta(\varphi) \in \operatorname{aut}(\widehat{T})$. A routine calculation shows $\delta(\varphi \psi)=\delta(\varphi) \delta(\psi)$, so $\delta: \operatorname{aut}\left(\sigma_{T}\right) \rightarrow \operatorname{aut}(\widehat{T})$ is a homomorphism.

QUESTION 6.1. Is the dimension representation $\delta: \operatorname{aut}\left(\sigma_{T}\right) \rightarrow \operatorname{aut}(\widehat{T})$ always surjective?

If $U$ is an integral matrix commuting with $T$, we define $\widehat{U}$ on $\mathcal{G}_{T}$ by $\widehat{U}([v])=[v U]$.

LEMMA 6.2. Suppose $U$ and $V$ are nonnegative integral matrices so that $T=$ $U V=V U$ is an elementary strong shift equivalence of $T$ to itself. Then there is a $\varphi \in \operatorname{aut}\left(\sigma_{T}\right)$ with $\delta(\varphi)=\widehat{U}$.

PROOF. This is mainly a matter of checking that the induced automorphism defined by Williams [Wi] acts correctly on beams. 
Suppose $T$ is $r \times r$. Let $W=\left[\begin{array}{ll}0 & U \\ V & 0\end{array}\right]$, so $W^{2}=\left[\begin{array}{ll}T & 0 \\ 0 & T\end{array}\right]$, indexed by states $\{0,1, \ldots, 2 r-1\}$. Then $B_{2}\left(\sigma_{W}\right)$ is the disjoint union of the set $B_{U, V}$ of paths beginning and ending in $\{0, \ldots, r-1\}$ and the set $B_{V, U}$ beginning and ending in $\{r, \ldots, 2 r-1\}$. Thus $X_{W^{2}}$ is the disjoint union of $X_{U, V}$ defined from $B_{U, V}$ and $X_{V, U}$ defined from $B_{V, U}$. Note that $\sigma_{W}$ switches these sets. From the form of $W^{2}$, it follows that there are bijections $\theta_{U, V}: B_{1}\left(\sigma_{T}\right) \rightarrow B_{U, V}$ and $\theta_{V, U}: B_{1}\left(\sigma_{T}\right) \rightarrow B_{V, U}$ that respect initial and terminal states $\bmod r$. These induce 1-block conjugacies, denoted by the same symbol, $\theta_{U, V}:\left(X_{T}, \sigma_{T}\right) \rightarrow\left(X_{U, V}, \sigma_{W}^{2}\right)$ and $\theta_{V, U}:\left(X_{T}, \sigma_{T}\right) \rightarrow$ $\left(X_{V, U}, \sigma_{W}^{2}\right)$. Let $\varphi=\theta_{V, U}^{-1} \sigma_{W} \theta_{U, V} \in \operatorname{aut}\left(\sigma_{T}\right)$.

We compute the action of $\varphi$ on a left-infinite ray $x(-\infty, n]^{*}$. Suppose $x_{n}$ has terminal state $i$. Then $\theta_{U, V}$ maps this to a ray in $X_{U, V}$ ending with state $i$. Next $\sigma_{W}$ maps this to a union of rays, with $U_{i j}$ of them ending in state $j$. Finally, $\theta_{V, U}^{-1}$ maps each ray ending in state $j$ to one in $X_{T}$ ending in state $j$. Passing to the action of $\varphi$ on $\mathcal{G}_{T}$, we see the standard unit vector $e_{i}$ is mapped by $\delta(\varphi)$ to $\sum_{j=1}^{r} U_{i j} e_{j}$. Thus $\delta(\varphi)=\widehat{U}$.

Note that aut $\left(\sigma_{T}\right)$ is naturally a subgroup of aut $\left(\sigma_{T}^{n}\right)$. Furthermore, the restriction of $\delta: \operatorname{aut}\left(\sigma_{T}^{n}\right) \rightarrow \operatorname{aut}\left(\widehat{T}^{n}\right)$ to $\operatorname{aut}\left(\sigma_{T}\right)$ coincides with the definition of $\delta$ of $\operatorname{aut}\left(\sigma_{T}\right)$.

Proposition 6.3. Suppose $\Phi \in \operatorname{aut}(\widehat{T})$. Then for all sufficiently large $n$, there is a $\varphi \in \operatorname{aut}\left(\sigma_{T}^{n}\right)$ with $\delta(\varphi)=\Phi$.

PROOF. Let $T$ be $r \times r$ acting on $\mathbb{Q}^{r}$. Then $R=T^{r} \mathbb{Q}^{r}$ is the eventual range of $T$, and $K=\operatorname{ker} T^{r}$ is its eventual kernel. Hence $\mathbb{Q}^{r}=R \oplus K$. As before, we may consider $\mathcal{G}_{T}$ as embedded in $R$. Since $\mathcal{G}_{T}$ is torsion-free, the automorphism $\Phi$ extends to a nonsingular $\mathbb{Q}$-linear map $W$ of $\mathcal{G}_{T} \otimes \mathbb{Q}=R$. If $0_{K}$ denotes the zero map on $K$, and $U_{0}$ is the matrix for $0_{K} \oplus W$ with respect to the standard basis, then $U_{0}=0_{K} \oplus W$ has rational entries, and $\widehat{U}_{0}=\Phi$. Let $V_{0}=0_{K} \oplus W^{-1}$. Both $U_{0}$ and $V_{0}$ have rows in $\mathcal{G}_{T}^{+}$, so for $k$ and $n-k$ large enough, $U=U_{0} T^{k}$ and $V=V_{0} T^{n-k}$ have $\mathbb{Z}^{+}$entries. Thus $U V=\left(0_{K} \oplus I_{R}\right) T^{n}=T^{n}=V U$ is an elementary strong shift equivalence of $T^{n}$ to itself. By Lemma 6.1 , there is a $\psi \in \operatorname{aut}\left(\sigma_{T}^{n}\right)$ with $\delta(\psi)=\widehat{U}$. Put $\varphi=\psi \sigma_{T}^{-k}$. Then $\delta(\varphi)=\delta(\psi) \delta\left(\sigma_{T}^{-k}\right)=\widehat{U}_{0} \widehat{T}^{k} \widehat{T}^{-k}=\widehat{U}_{0}=\Phi$.

To use Proposition 6.3, we will establish some results about finite generation of $\operatorname{aut}(\widehat{T})$. In what follows, aut $\left(\mathcal{G}_{T}, \widehat{T}\right)$ refers to the group of those automorphisms of $\mathcal{G}_{T}$ commuting with $\widehat{T}$, not necessarily preserving the positive cone. If $U$ has nonzero eigenvalues $\lambda_{1}, \ldots, \lambda_{k}$, let $\chi_{U}^{\times}(t)$ denote $\prod_{j=1}^{k}\left(t-\lambda_{j}\right)$.

LEMMA 6.4. Suppose $\chi_{U}^{\times}$is irreducible. Then aut $\left(\mathcal{G}_{U}, \widehat{U}\right)$ is finitely generated and abelian.

PrOOF. By passing to the eventual range of $U$, we may suppose $U$ is nonsingular. Let $U$ be $r \times r$, and let $\lambda$ be an eigenvalue of $U$. By a theorem of Taussky [ $\mathbf{T}$ ], there is an ideal $J \subset \mathbb{Z}[\lambda]$ so that $\left(\mathbb{Z}^{r}, U\right) \cong\left(J, M_{\lambda}\right)$, where $M_{\lambda}$ denotes multiplication by $\lambda$. Hence $\left(\mathcal{G}_{U}, \widehat{U}\right) \cong\left(\mathbb{Z}[1 / \lambda] J, M_{\lambda}\right)$. For more on this correspondence, see [BMT, Chapter 5]. Under this isomorphism, an automorphism $\Phi$ of $\widehat{U}$ corresponds to an automorphism of $\mathbb{Z}[1 / \lambda] J$ commuting with $M_{\lambda}$, i.e. a $\mathbb{Z}[1 / \lambda]$-module isomorphism. Since the quotient field of $\mathbb{Z}[1 / \lambda]$ is $\mathbb{Q}(\lambda)$, there is an $\alpha \in \mathbb{Q}(\lambda)$ so that $\Phi$ corresponds to the restriction of $M_{\alpha}$ on $\mathbb{Z}[1 / \lambda] J$. Let $S$ be the set of prime divisors of the ideal 
generated by $\lambda$, and $O_{S}$ denote the ring of $S$-integral elements in $\mathbb{Q}(\lambda)$. Since both $M_{\alpha}$ and $M_{\alpha^{-1}}$ are automorphisms of $\mathbb{Z}[1 / \lambda] J$, it follows that $\alpha$ is in the unit group $O_{S}^{\times}$of $O_{S}$. By the Dirichlet unit theorem [We, Theorem 5-3-10], the group $O_{S}^{\times}$of units is a finitely generated abelian group. Thus aut $(\widehat{U})$ corresponds to a subgroup of $O_{S}^{\times}$, so is also finitely generated and abelian.

REMARK. The Dirichlet unit theorem shows that aut $\left(\mathcal{G}_{U}, \widehat{U}\right)$ is the product of a finite cyclic group and a free abelian group on $e+r+s-1$ generators, where $\mathbb{Q}(\lambda)$ has $r$ real and $2 s$ complex embeddings, and the factorization of the principal ideal generated by $\lambda$ uses $e$ distinct primes.

PROPOSITION 6.5. If all the nonzero eigenvalues of $T$ are simple, then $\operatorname{aut}(\widehat{T})$ is finitely generated and abelian.

PROOF. Factor $\chi_{T}(t)=t^{m} p_{1}(t) \cdots p_{k}(t)$, where the $p_{j}(t)$ are distinct irreducibles. If $T$ is $r \times r$, then $\mathbb{Q}^{r}$ is the direct sum of the eventual kernel of $T$ and the rational subspaces $R_{j}$ corresponding to the $p_{j}(t)$. Let $\mathcal{G}_{j}=\mathcal{G}_{T} \cap R_{j}$, and $\widehat{T}_{j}=\widehat{T} \mid \mathcal{G}_{j}$. Suppose $\Phi \in \operatorname{aut}(\widehat{T})$. As before, $\Phi$ extends to a $\mathbb{Q}$-linear map of $\mathbb{Q}^{r}$ which is invertible on $\bigoplus_{j=1}^{k} R_{j}$. Since the $p_{j}(t)$ are distinct and irreducible, each $R_{j}$ is $\Phi$-invariant. It follows $\left.\Phi\right|_{\mathcal{G}_{j}}$ is an automorphism of $\left(\mathcal{G}_{j}, \widehat{T}_{j}\right)$. Hence the map $\left.\Phi \mapsto \bigoplus_{j=1}^{k} \Phi\right|_{\mathcal{G}_{j}}$ takes aut $\left(\mathcal{G}_{T}, \widehat{T}\right)$ to $\bigoplus_{j=1}^{k}$ aut $\left(\mathcal{G}_{j}, \widehat{T}_{j}\right)$. Since $\bigoplus_{j=1}^{k} \mathcal{G}_{j}$ has finite index in $\mathcal{G}_{T}$, this mapping is injective. By using an integral basis for $\mathbb{Z}^{r} \cap R_{j}$, the map $\widehat{T}_{j}$ is seen to be a dimension group automorphism, so aut $\left(\mathcal{G}_{j}, \widehat{T}_{j}\right)$ is finitely generated abelian by Lemma 6.4. This proves aut $\left(\mathcal{G}_{T}, \widehat{T}\right)$ is finitely generated abelian. The group aut $(\widehat{T})$ of order-preserving automorphisms is therefore also finitely generated abelian.

LEMMA 6.6. Suppose the nonzero eigenvalues of $T$ are simple. If $T$ does not have distinct eigenvalues whose ratio is an nth root of unity, then $\operatorname{aut}\left(\widehat{T}^{n}\right)=\operatorname{aut}(\widehat{T})$.

Proof. As noted above, aut $(\widehat{T}) \subset \operatorname{aut}\left(\widehat{T}^{n}\right)$ is trivial.

Using the notations from the proof of Proposition 6.5, we see the hypotheses on the eigenvalues of $T$ mean that the spectra of $\left.T^{n}\right|_{R_{j}}$ are disjoint for $1 \leq j \leq k$. Thus any $\Phi \in \operatorname{aut}\left(\widehat{T}^{n}\right)$ extends to a $\mathbb{Q}$-linear map under which the $R_{j}$ are invariant. Since the eigenvalues of $T^{n}$ on $R_{j}$ are distinct, and $\Phi$ commutes with $T^{n}$ on $R_{j}$, it follows by linear algebra that $\Phi$ commutes with $T$ on $R_{j}$, completing the proof.

EXAMPLE 6.7. An aperiodic matrix $T$ for which aut $\left(\widehat{T}^{2}\right)$ is larger than $\operatorname{aut}(\widehat{T})$, but both are finitely generated.

Let

$$
T=\left[\begin{array}{lll}
5 & 2 & 2 \\
4 & 1 & 4 \\
0 & 6 & 3
\end{array}\right]
$$

Then over $\mathbb{Z}[1 / 3]$ we find

$$
V^{-1} T V=U=\left[\begin{array}{ccc}
9 & 0 & 0 \\
0 & 3 & 0 \\
0 & 0 & -3
\end{array}\right], \quad \text { where } V=\left[\begin{array}{ccc}
1 & 1 & 0 \\
1 & 0 & 1 \\
1 & -1 & -1
\end{array}\right] \text {. }
$$

Since the eigenvalues of $U$ are distinct, we obtain $\mathcal{G}_{U} \cong \mathbb{Z}[1 / 3]^{3}$, and aut $\left(\mathcal{G}_{U}, \widehat{U}\right) \cong$ $G L(1, \mathbb{Z}[1 / 3])^{3} \cong[\mathbb{Z} \oplus(\mathbb{Z} / 2 \mathbb{Z})]^{3}$. Since order-preserving automorphisms of $\mathcal{G}_{T}$ just 
need to preserve the positive dominant eigendirection, aut $\left(\mathcal{G}_{T}, \mathcal{G}_{T}^{+}, \widehat{T}\right)$ is a subgroup of index 2 in aut $\left(\mathcal{G}_{T}, \widehat{T}\right)$. The conjugacy of $T$ and $U$ takes place over $\mathbb{Z}[1 / 3]$, so $\operatorname{aut}\left(\mathcal{G}_{U}, \widehat{U}\right) \cong \operatorname{aut}\left(\mathcal{G}_{T}, \widehat{T}\right)$. Hence $\operatorname{aut}(\widehat{T}) \cong \mathbb{Z}^{3} \oplus(\mathbb{Z} / 2 \mathbb{Z})^{2}$.

On the other hand, $U^{2}$ has a repeated eigenvalue of $3^{2}$, and any automorphism of the corresponding subgroup of $\mathcal{G}_{U}$ extends to one of $\mathcal{G}_{U}$. Thus

$$
\operatorname{aut}\left(\mathcal{G}_{U}, \widehat{U}^{2}\right) \cong G L(1, \mathbb{Z}[1 / 3]) \oplus G L(2, \mathbb{Z}[1 / 3]) \text {. }
$$

As before, we conclude aut $\left(\widehat{T}^{2}\right) \cong \mathbb{Z} \oplus G L(2, \mathbb{Z}[1 / 3])$, which is larger than $\operatorname{aut}(\widehat{T})$.

We remark that by using elementary matrix operations, one can show that $G L(2, \mathbb{Z}[1 / 3])$, and hence aut $\left(\widehat{T}^{2}\right)$, is a finitely generated nonabelian group.

Assembling the pieces, we now state the main result of this section.

THEOREM 6.8. Suppose the nonzero eigenvalues of $T$ are simple. If no ratio of distinct eigenvalues is a root of unity, then for all sufficiently large $n$ we have that $\delta: \operatorname{aut}\left(\sigma_{T}^{n}\right) \rightarrow \operatorname{aut}\left(\widehat{T}^{n}\right)=\operatorname{aut}(\widehat{T})$ is surjective. If some ratios of eigenvalues are roots of unity, the conclusion still holds for infinitely many $n$.

PrOOF. Let us first suppose no ratio of distinct eigenvalues is a root of unity. By Lemma 6.6, we have aut $\left(\widehat{T}^{n}\right)=\operatorname{aut}(\widehat{T})$. By Proposition 6.5 , aut $(\widehat{T})$ is finitely generated. Using Proposition 6.3, for all sufficiently large $n$, each generator is in $\delta\left(\operatorname{aut}\left(\sigma_{T}^{n}\right)\right)$, implying the conclusion.

If some ratios of distinct eigenvalues are roots of unity, the preceding argument still holds for all $n$ relatively prime to the orders of these roots of unity.

We conclude with an example to show that finite generation of aut $(\widehat{T})$ does not generally hold.

EXAMPLE 6.9. An aperiodic matrix $T$ with aut $(\widehat{T})$ not finitely generated.

We are grateful to Gopal Prasad for the idea behind the following. Let

$$
T=\left[\begin{array}{lll}
5 & 2 & 2 \\
4 & 4 & 1 \\
0 & 3 & 6
\end{array}\right]
$$

Then $T$ is conjugate over $\mathbb{Z}[1 / 3]$ to

$$
U=V^{-1} T V=\left[\begin{array}{lll}
9 & 0 & 0 \\
0 & 3 & 0 \\
0 & 3 & 3
\end{array}\right], \quad \text { where } V=\left[\begin{array}{ccc}
1 & 1 & 0 \\
1 & 0 & 1 \\
1 & -1 & -1
\end{array}\right] .
$$

As in the argument for Example 6.7, aut $\left(\mathcal{G}_{U}\right) \cong G L(1, \mathbb{Z}[1 / 3]) \times H$, where $H$ is the group of automorphisms of $\mathbb{Z}[1 / 3]^{2}$ commuting with $W=\left[\begin{array}{ll}3 & 0 \\ 3 & 3\end{array}\right]$. By analogy with the $K A N$ decomposition of Lie groups, it is easy to see that $H \cong K \times A \times N$, where $K=\{ \pm 1\}, A=\left\{3^{n} I: n \in \mathbb{Z}\right\}$, and

$$
N=\left\{\left[\begin{array}{ll}
1 & 0 \\
\alpha & 1
\end{array}\right]: \alpha \in \mathbb{Z}[1 / 3]\right\}
$$

Now $N$ is isomorphic to the additive group $\mathbb{Z}[1 / 3]$, which is not finitely generated. Thus $H$ is not finitely generated, so aut $(\widehat{T}) \cong \mathbb{Z} \times H$ is also not finitely generated.

The following question appears to us basic to understanding the structure of $\operatorname{aut}\left(\sigma_{T}\right)$. 
QUESTION 6.10. If $T$ is aperiodic, is the kernel of the dimension representation of $\operatorname{aut}\left(\sigma_{T}\right)$ generated by elements of finite order?

This question is especially pertinent to understanding the action of aut $\left(\sigma_{T}\right)$ on the periodic points of $\sigma_{T}$ (see $\S 7$ and [BK]). It is a natural generalization of an older one, which to the best of our knowledge was first explicitly conjectured by $\mathrm{F}$. Rhodes in correspondence with G. Hedlund.

CONJECTURE 6.11 (F. RHODES). The automorphism group of the 2-shift is generated by the 2-shift and involutions.

7. Induced action on periodic points. Each automorphism of $\sigma_{T}$ maps a periodic point to another with the same period. This action of $G$ on periodic points was studied by Boyle and Krieger $[\mathbf{B K}]$. We begin by discussing a fundamental question about this action, and give a partial answer in Theorem 7.2. We then discuss several representations of $G$ provided via periodic points, and conclude with remarks about some related topologies on $G$.

Let $P_{n}=P_{n}\left(\sigma_{T}\right)$ denote the fixed points of $\sigma_{T}^{n}$, and $Q_{n}=Q_{n}\left(\sigma_{T}\right)$ be those points with least $\sigma_{T}$-period $n$. Since $Q_{n}$ is $\sigma_{T}$-invariant, we can define aut $\left(Q_{n}, \sigma_{T}\right)$ to be the group of bijections of $Q_{n}$ commuting with $\sigma_{T}$. Let $F_{n}=\bigcup_{j=1}^{n} Q_{j}$.

QUESTION 7.1. When is an automorphism in aut $\left(F_{n}, \sigma_{T}\right)$ the restriction of one in $\operatorname{aut}\left(X_{T}, \sigma_{T}\right)$ ?

This question is the natural generalization of a long-standing problem of R. F. Williams, namely when can fixed points be switched by an automorphism. The latter problem, posed originally as a potential refinement of shift equivalence, has withstood serious attacks for a number of years. Example 7.3 below provides a concrete case of a shift of finite type having two fixed points which no composition of finite-order elements can switch. It cannot be ruled out at present that the answer to Question 7.1 is "always".

THEOREM 7.2. Let $\sigma_{T}$ be a mixing shift of finite type. There is an $n_{0}(T)$ so that if $n \geq n_{0}$ and $x, y \in Q_{n}\left(\sigma_{T}\right)$ have disjoint orbits, there is a composition $\varphi$ of involutions in aut $\left(\sigma_{T}\right)$ so that $\varphi x=y, \varphi y=x$, and $\varphi$ fixes all points whose orbit has length $\leq n$ and does not contain $x$ or $y$.

PROOF. The proof elaborates ideas from [BK], where this is proved for full shifts with $n_{0}=1$. Let us first describe some convenient notation. Shorten $\sigma_{T}$ to $\sigma, X_{T}$ to $X, B_{n}\left(X_{T}\right)$ to $B_{n}$, and $B\left(X_{T}\right)$ to $B$. If $A=a_{0} \cdots a_{n-1} \in B_{n}$, let $A[i, j]$ denote $a_{i} \cdots a_{j}$. If $A^{2} \in B_{2 n}$, let $A^{\infty}$ denote the point $x \in X$ with $x_{i}=A[i \bmod n]$. If $0 \leq i<n$, let $\sigma^{i}(A)$ be the cyclic permutation $A[i, n-1] A[0, i-1]$ of $A$. Then $\sigma^{i}\left(A^{\infty}\right)=\left(\sigma^{i} A\right)^{\infty}$.

First suppose $A$ and $B$ are distinct blocks in $B_{n}$ with $A^{2} B^{2} A^{2} \in B$. Although $(A B)^{\infty}$ need not have least period $2 n$, we claim there are cyclic permutations $\sigma^{i}(A), \sigma^{i}(B)$ so that $\left(\sigma^{i}(A) \sigma^{i}(B)\right)^{\infty} \in Q_{2 n}$. To prove this, suppose $(A B)^{\infty}$ has least period $2 n / m<2 n$, so $A B=C^{m}$ with $m \geq 3$ and odd since $A \neq B$. Thus $|C|$ is even, so write $C=D E$ with $|D|=|E|$ and $D \neq E$. Thus $A=D(E D)^{q}$ and $B=E(D E)^{q}$ with $q=\frac{1}{2}(m-1)$. Let $A_{1}=\sigma^{|D|}(A)$ and $B_{1}=\sigma^{|D|}(B)$, and put $F=A_{1} B_{1}=(E D)^{q-1} E D D D E(D E)^{q-1} E$. Suppose $F^{\infty}$ has period $k<2 n$. Since 
$D \neq E$, we conclude $k<n$, hence

$$
k \leq \frac{2 n}{3}=\frac{4 q+2}{3}|D| \leq 2 q|D| .
$$

Now choose $j \geq 1$ such that $|D| \leq j k \leq 2 q|D|$, and translate the central $D D D$ block in $F$ by $j k$ to the left. It follows that this block contains either $D E$ or $E D$ as a subblock, implying $D=E$, a contradiction. Hence $F^{\infty} \in Q_{2 n}$, verifying our claim. Note that the cyclic permutations $A_{1}$ and $B_{1}$ retain the property that $A_{1}^{2} B_{1}^{2} A_{1}^{2} \in B$, so $A_{1}^{\infty}, B_{1}^{\infty}$, and $\left(A_{1} B_{1}\right)^{\infty}$ are allowed points in $X$.

If $x, y \in Q_{n}$, say that $\varphi \in G$ switches $x$ and $y$ if $\varphi x=y, \varphi y=x$, and $\varphi$ fixes all points whose $\sigma$-orbit has length $\leq n$ and does not contain $x$ or $y$. We shall write in this case $\varphi: x \leftrightarrow y$. Suppose now that $A, B \in B_{n}$ with $A^{2}, B^{2} \in B$ and $A^{\infty}, B^{\infty} \in Q_{n}$ defining disjoint orbits with $A[i]=B[i]$ for some $0 \leq i<n$. We will construct an involution $\varphi_{A B}: A^{\infty} \leftrightarrow B^{\infty}$. First replace $A$ by $\sigma^{i} A$ and $B$ by $\sigma^{i} B$, so now $A^{2} B^{2} A^{2} \in B$. By the above, we can further replace $A$ by $\sigma^{j} A$ and $B$ by $\sigma^{j} B$ so that still $A^{2} B^{2} A^{2} \in B$ and also $(A B)^{\infty} \in Q_{2 n}$. To define $\varphi_{A B}$, let a frame be a word in $\{A, B\}^{5}$. If $z[i-2 n, i+3 n-1]$ is a frame, define

$$
\varphi_{A B}(z)[i, i+n-1]= \begin{cases}B & \text { if } x[i, i+n-1]=A, \\ A & \text { if } x[i, i+n-1]=B,\end{cases}
$$

and require $\varphi_{A B}$ to have no other effect. To see that $\varphi_{A B}$ is well-defined, suppose the contrary. Then there are two frames $C$ and $D$ with $C[-2 n, 3 n-1-k]=$ $D[-2 n+k, 3 n-1]$ for some $k$ with $0<k<n$. Since $A^{\infty} \in Q_{n}$, it follows that $A$ occurs in $A A$ only in the initial and terminal halves, and also $B$ does not occur in $A A$. Thus $C$ and $D$ have the form $A B A B A$ or $B A B A B$. The overlapping of $C$ and $D$ force $(A B)^{\infty}$ to have period $<2 n$, contradicting $(A B)^{\infty} \in Q_{2 n}$. Thus $\varphi_{A B}$ is well-defined. Clearly it preserves frames. It follows that $\varphi_{A B}$ is an involution in $G$, and obviously it switches $A^{\infty}$ with $B^{\infty}$.

To complete the proof, let $l>0$ be a transition length for $\sigma_{T}$, so that $\left(T^{l}\right)_{i j}>0$ for all $i, j \in \mathcal{L}$. There is an $n_{0}=n_{0}(T)$ so that for all $a, b \in \mathcal{L}$ and $n \geq n_{0}$, there are at least $3 n$ blocks $D$ in $B_{n}$ satisfying $D[0]=a, D[l]=b$, and $D^{\infty} \in Q_{n}$. This follows by counting, since the number of $n$-blocks satisfying the first two conditions is at least $\kappa \lambda_{T}^{n}$ for suitable $\kappa>0$, while the number of $n$-blocks $D$ with $D^{\infty}$ having period $<n$ is no more than $\sum_{d \mid n, d<n}\left|B_{d}\right|=O\left(\lambda_{T}^{n / 2}\right)$. Now fix $n \geq n_{0}(T)$, and suppose $x, y \in Q_{n}$ have distinct orbits. Put $A=x[0, n-1]$ and $B=y[0, n-1]$. By the choice of $n_{0}$, there is $C \in B_{n}$ with $C[0]=A[0], C[l]=B[l], C^{\infty} \in Q_{n}$, and the orbit of $C^{\infty}$ missing $x$ and $y$. By the above, $\varphi_{A C}: A^{\infty} \leftrightarrow C^{\infty}$ and $\varphi_{B C}: B^{\infty} \leftrightarrow C^{\infty}$. Then $\varphi=\varphi_{A C} \varphi_{B C} \varphi_{A C}: A^{\infty} \leftrightarrow B^{\infty}$, and no other orbits of length $\leq n$ are affected.

Theorem 7.2, with a smaller estimate for $n_{0}(T)$, has been proved independently by M. Nasu [N].

The following example shows it is possible to have periodic orbits that cannot be switched by products of finite-order automorphisms.

EXAMPLE 7.3. A mixing $\sigma_{T}$ with exactly two fixed points that are also fixed by every finite-order automorphism of $\sigma_{T}$.

Let

$$
T=\left[\begin{array}{lll}
1 & 1 & 0 \\
0 & 0 & 1 \\
3 & 0 & 1
\end{array}\right]
$$


and $Q_{1}\left(\sigma_{T}\right)=\{x, y\}$, where $x=0^{\infty}$ and $y=2^{\infty}$. Suppose $\psi \in G$ with $\psi^{k}=I$ and $\psi: x \leftrightarrow y$. Now $\left(\psi \sigma_{T}\right)^{k}=\sigma_{T}^{k}$, so $\psi \sigma_{T}$ is a root of a mixing shift of finite type, hence itself is a mixing shift of finite type ([L1, Theorem 8] or [BK, Lemma 2.5]). Since the characteristic polynomial of $T$ is irreducible over $\mathbb{Q}$, it follows that $\sigma_{T}$ and $\psi \sigma_{T}$ have equal $\varsigma$-functions. But $x \in Q_{2}\left(\psi \sigma_{T}\right)$, while $Q_{2}\left(\sigma_{T}\right)$ is empty. This contradiction shows such a $\psi$ cannot exist. Furthermore, $\sigma_{T}$ has no roots. For suppose $\psi^{k}=\sigma_{T}$. Then as before $\psi$ is a shift of finite type, say $\psi=\sigma_{U}$. But then the product of the nonzero eigenvalues of $U$ is $3^{1 / k}$, contradicting integrality of this product. In this example, no combination of marker constructions and roots of the shift can switch $x$ and $y$, and it remains open whether this is possible.

Recall that $P_{n}=P_{n}\left(\sigma_{T}\right)$ denotes the fixed points of $\sigma_{T}^{n}$, and that $Q_{n}=Q_{n}\left(\sigma_{T}\right)$ is the set of points with least $\sigma_{T}$-period $n$. Clearly each $\varphi \in G$ restricts to a permutation $\pi_{n}(\varphi)=\left.\varphi\right|_{Q_{n}} \in \operatorname{aut}\left(Q_{n}, \sigma_{T}\right)$. If $Q_{n}=\varnothing$, set aut $\left(Q_{n}, \sigma_{T}\right)$ to the trivial group, so then $\pi_{n}$ is also trivial. Call

$$
\pi(\varphi)=\left(\pi_{1}(\varphi), \pi_{2}(\varphi), \ldots\right) \in \prod_{n=1}^{\infty} \operatorname{aut}\left(Q_{n}, \sigma_{T}\right)
$$

the periodic point representation of $\varphi \in G$. The proof of Theorem 3.1 shows that it is faithful.

PROPOSITION 7.4. The periodic point representation $\pi$ of $G$ is faithful.

The next two representations are derived from $\pi$. Let $\Sigma=\left\{\sigma_{T}^{n}: n \in \mathbb{Z}\right\}$, and $\mathcal{Q}_{n}=Q_{n} / \Sigma$ be the set of orbits of length $n$. If $\varphi \in G$, then $\pi_{n}(\varphi)$ commutes with $\pi_{n}(\sigma)$, so induces a permutation $\rho_{n}(\varphi) \in \operatorname{aut}\left(\mathcal{Q}_{n}, \sigma_{T}\right)$. Call

$$
\rho(\varphi)=\left(\rho_{1}(\varphi), \rho_{2}(\varphi), \ldots\right) \in \prod_{n=1}^{\infty} \operatorname{aut}\left(\mathcal{Q}_{n}, \sigma_{T}\right)
$$

the periodic orbit representation of $\varphi \in G$, where again we use the convention that the symmetric group of the empty set is the trivial group. Clearly $\rho\left(\sigma^{k}\right)=I$, but $\Sigma$ exhausts the kernel of $\rho$.

PROPOSITION 7.5. The periodic orbit representation $\rho$ of $G$ is faithful on $G / \Sigma$.

ProOF. Suppose $\rho_{n}(\varphi)=I$ for $n \geq 1$. By Theorem 2.5 of $[\mathbf{B K}]$, it follows that $\varphi \in \Sigma$.

We next prove that the range of $\rho$ is large, in that its closure in the compact group $\prod_{n=1}^{\infty} \operatorname{aut}\left(\mathcal{Q}_{n}, \sigma_{T}\right)$ is a subgroup of finite index.

THEOREM 7.6. The closure of $\rho(G)$ in $\prod_{n=1}^{\infty}$ aut $\left(\mathcal{Q}_{n}, \sigma_{T}\right)$ is a subgroup of finite index.

PROOF. Let $n_{0}=n_{0}(T)$ from Theorem 7.2. We assume without loss that $\left|\mathcal{Q}_{n}\right| \geq 1$ for $n \geq n_{0}$. Suppose $n \geq n_{0}$, and let $\theta_{j} \in \operatorname{aut}\left(\mathcal{Q}_{j}, \sigma_{T}\right)$ for $1 \leq j \leq n$ with $\theta_{j}=I$ if $1 \leq j \leq n_{0}$. We show there is a $\varphi \in G$ with $\rho_{j}(\varphi)=\theta_{j},(1 \leq j \leq n)$. It will follow that $\overline{\rho(G)}$ is a finite union of cosets of $\prod_{n=n_{0}}^{\infty}$ aut $\left(\mathcal{Q}_{n}, \sigma_{T}\right)$. Set $\varphi_{1}=I$, and suppose inductively that $\varphi_{j} \in G$ has been found with $\rho_{i}\left(\varphi_{j}\right)=\theta_{j}$ for $1 \leq i \leq j$. Since $\rho_{j+1}\left(\varphi_{j}\right)^{-1} \theta_{j+1} \in \operatorname{aut}\left(\mathcal{Q}_{j+1}, \sigma_{T}\right)$ is a product of transpositions, by Theorem 7.2 there is a product $\psi_{j+1}$ of involutions in $G$ with $\rho_{i}\left(\psi_{j+1}\right)=I$ for $1 \leq i \leq j$ and 
$\rho_{j+1}\left(\psi_{j+1}\right)=\rho_{j+1}\left(\varphi_{j}\right)^{-1} \theta_{j+1}$. Set $\varphi_{j+1}=\varphi_{j} \psi_{j+1}$ and continue. Then $\varphi=\varphi_{n}$ has the required properties.

One implication of this density result is another proof of Ryan's theorem [Ry2] on the center of $G$. This proof was obtained jointly with W. Krieger.

THEOREM 7.7 (RYAN). The center of $G$ is $\Sigma$.

ProOF. Choose $n_{1} \geq n_{0}(T)$ so that if $n \geq n_{1}$, then $\left|\mathcal{Q}_{n}\right| \geq 3$. If $\varphi$ commutes with every element in $G$, then by Theorem 4.5 its restriction $\rho_{n}(\varphi)$ to orbits commutes with all of aut $\left(\mathcal{Q}_{n}, \sigma_{T}\right)$ for $n \geq n_{1}$. Hence $\rho_{n}(\varphi)=I$ for $n \geq n_{1}$, so by Theorem 2.5 in $[\mathbf{B K}]$ it follows that $\varphi \in \Sigma$.

THEOREM 7.8. The groups $G, G / \Sigma$, and $G /[G, G]$ are not finitely generated.

PROOF. We show each factors onto an arbitrarily large product of two-element groups, which shows each is not finitely generated. By Theorem 4.5 there is an $n_{1} \geq n_{0}(T)$ so that for $n \geq n_{1}$ we have $\left|\mathcal{Q}_{n}\right| \geq 2$ and that $\left(\rho_{n_{1}}, \ldots, \rho_{n}\right)$ maps $G$ onto $\prod_{j=n_{1}}^{n} \operatorname{aut}\left(\mathcal{Q}_{j}, \sigma_{T}\right)$. Denoting the sign of a permutation $\rho$ by $\operatorname{sgn} \rho \in\{ \pm 1\}$, it follows that $\left(\operatorname{sgn} \rho_{n_{1}}, \ldots, \operatorname{sgn} \rho_{n}\right)$ maps $G$ onto $\{ \pm 1\}^{n-n_{1}}$. This mapping factors through $G \rightarrow G / \Sigma$ and $G \rightarrow G /[G, G]$, completing the proof.

We now recall the gyration function $g$ introduced in [BK]. To define $g$, let $\varphi \in G$, and from each orbit $\gamma \in \mathcal{Q}_{n}$ pick an element $x_{\gamma} \in \gamma$. Since $\varphi\left(x_{\gamma}\right) \in \varphi(\gamma)$, there is an integer $n(\gamma, \varphi)$ defined modulo $n$ so that $\varphi\left(x_{\gamma}\right)=\sigma^{n(\gamma, \varphi)} x_{\varphi \gamma}$. Put $g(\varphi)(n)=$ $g(\varphi, \sigma)(n)=\sum_{\gamma \in \mathcal{Q}_{n}} n(\gamma, \varphi)$, where by convention an empty sum is 0 . The value of $g(\varphi)(n)$ is shown in [BK] to be independent of the choice of the $x_{\gamma}$, and also $g(\varphi \psi)(n) \equiv g(\varphi)(n)+g(\psi)(n) \bmod n$. Call

$$
g(\varphi)=(g(\varphi)(1), g(\varphi)(2), \ldots) \in \prod_{n=1}^{\infty} \mathbb{Z} / n \mathbb{Z}
$$

the gyration function of $\varphi \in G$, and call the homomorphism $\varphi \mapsto g(\varphi)$ the gyration representation. This function is studied in detail by Boyle and Krieger [BK]

The periodic orbit and gyration representations are related. If $\theta \in \operatorname{aut}\left(Q_{n}, \sigma_{T}\right)$ commutes with $\pi_{n}\left(\sigma_{T}\right)=\left.\sigma\right|_{Q_{n}}$, its gyration number $g(\theta) \in \mathbb{Z} / n \mathbb{Z}$ is defined as above. Also, let $\rho(\theta)$ be the permutation induced by $\theta$ on the orbit space $\mathcal{Q}_{n}=$ $Q_{n} / \Sigma$. Call a sequence $\left(\theta_{1}, \ldots, \theta_{n}\right)$ with $\theta_{j} \in \operatorname{aut}\left(Q_{j}, \sigma_{T}\right)$ consistent if whenever $2^{m} q \leq N$ with $q$ odd, then

$$
g\left(\theta_{2^{m} q}\right) \equiv\left\{\begin{array}{lll}
0 & \bmod 2^{m} q & \text { if } \prod_{j=1}^{m-1} \operatorname{sgn} \rho\left(\theta_{2^{j} q}\right)=1, \\
2^{m-1} q & \bmod 2^{m} q & \text { if } \prod_{j=1}^{m-1} \operatorname{sgn} \rho\left(\theta_{2^{j} q}\right)=-1 .
\end{array}\right.
$$

In particular, if $m=0$ the empty product being 1 means $g\left(\theta_{q}\right) \equiv 0$. If $\sigma_{T}$ is a full shift, it is shown in [BK] that a necessary and sufficient condition for a sequence $\left(\theta_{1}, \ldots, \theta_{n}\right)$ with $\theta_{j} \in \operatorname{aut}\left(Q_{j}\left(\sigma_{T}\right), \sigma_{T}\right)$ to be the restriction $\theta_{j}=\pi_{j}(\varphi)$ of a product $\varphi$ of involutions is that it be consistent. Necessity was also shown for a broad class of $\sigma_{T}$. One consequence is that for a product $\varphi$ of involutions, $\operatorname{sgn} \rho(\varphi)$ and $g(\varphi)$ determine each other. This is significant since many marker constructions are involutions. We show a modified form of the sufficiency condition is true for general shifts of finite type. 
THEOREM 7.9. Let $\sigma_{T}$ be a mixing shift of finite type, and fix $N>n_{0}(T)$ with $n_{0}(T)$ as in Theorem 7.2. If $\theta_{j} \in \operatorname{aut}\left(Q_{j}\left(\sigma_{T}\right), \sigma_{T}\right)$ for $1 \leq j \leq N$ with $\theta_{j}=I$ if $1 \leq j \leq n_{0}$, and if $\left(\theta_{1}, \ldots, \theta_{N}\right)$ is consistent, then there is a product $\varphi$ of involutions with $\pi_{j}(\varphi)=\theta_{j}$ for $1 \leq j \leq N$.

PrOOF. We first observe that the involution $\varphi_{A B}$ in the proof of Theorem 7.2 can be constructed so that $\left(\pi_{1}\left(\varphi_{A B}\right), \ldots, \pi_{N}\left(\varphi_{A B}\right)\right)$ is consistent. Let $k=|A|$, and change the definition of "frame" in the construction from $\{A, B\}^{5}$ to $\{A, B\}^{2 N+1}$. The only points of period $\leq N$ affected by $\varphi_{A B}$ have period a multiple of $k$, and are concatenations of $A$ 's and $B$ 's. Let $\sigma_{2}$ be the 2-shift on $\{0,1\}$, and $\tau \in \operatorname{aut}\left(\sigma_{2}\right)$ be the 1-block map exchanging 0 and 1. Mapping $A \rightarrow 0$ and $B \rightarrow 1$ shows that $\rho_{n k}\left(\varphi_{A B}\right) \in \operatorname{aut}\left(\mathcal{Q}_{n k}\left(\sigma_{T}\right), \sigma_{T}\right)$ and $\rho_{n}(\tau) \in \operatorname{aut}\left(\mathcal{Q}_{n}\left(\sigma_{2}\right), \sigma_{2}\right)$ have the same sign. Furthermore, $g\left(\varphi_{A B}, \sigma_{T}\right)(2 n k) \equiv k g\left(\tau, \sigma_{2}\right)(2 n) \bmod 2 n k$. Since $\rho_{m}\left(\varphi_{A B}\right)=I$ if $m \not \equiv 0 \bmod k$, and $\left(\pi_{1}(\tau), \ldots, \pi_{N}(\tau)\right)$ is consistent from Lemma 3.3 of [BK], it follows that $\left(\pi_{1}\left(\varphi_{A B}\right), \ldots, \pi_{N}\left(\varphi_{A B}\right)\right)$ is also consistent.

To construct $\varphi$, assume inductively that $\varphi_{m}$ has been found with $\pi_{j}\left(\varphi_{m}\right)=\theta_{j}$ for $1 \leq j \leq m$ and so that $\left(\pi_{1}\left(\varphi_{m}\right), \ldots, \pi_{N}\left(\varphi_{m}\right)\right)$ is consistent. As in the proof of Lemma 3.7 of $[\mathbf{B K}]$, there is a product $\psi_{m}$ of involutions of the above type fixing points with period $\leq m$ such that $\pi_{m+1}\left(\psi_{m+1}\right)=\pi_{m+1}\left(\varphi_{m}^{-1} \theta_{m+1}\right)$ if the gyration number of $\varphi_{m}^{-1} \theta_{m+1}$ vanishes. However, this follows from consistency of the $\theta_{j}=\pi_{j}\left(\varphi_{m}\right)$.

Although $G$ is discrete is the compact-open mapping topology, there are at least two other natural topologies on $G$ making it a nondiscrete topological group. The first is the periodic point topology, defined as the weakest topology making the restriction homomorphisms $G \rightarrow \operatorname{aut}\left(Q_{n}, \sigma_{T}\right)$ continuous for all $n$. In this topology an automorphism is close to the identity it if fixes all points with period less than some large bound. The second is the profinite topology on $G$, which has as basic open sets the cosets of finite-index normal subgroups of $G$. Clearly the profinite topology refines the periodic point topology, and the proof of Proposition 7.4 shows both are Hausdorff. Do they coincide? The following example shows the answer in general is "no".

EXAMPLE 7.10. A mixing shift of finite type such that the profinite topology on its automorphism group strictly refines the periodic point topology.

Let

$$
T=\left[\begin{array}{lll}
0 & 1 & 1 \\
1 & 0 & 1 \\
1 & 1 & 1
\end{array}\right]
$$

indexed with states $a, b$, and $c$. We will use the dimension group representation of $G$ to define a homomorphism $\theta$ from $G$ to $\{ \pm 1\}$, and show that for every $n$ there is a $\varphi_{n} \in G$ fixing all points of period $\leq n$ for which $\theta\left(\varphi_{n}\right)=-1$. This will show that the profinite neighborhood $\operatorname{ker} \theta$ of $I$ in $G$ contains no periodic point neighborhood of $I$.

Now $T$ has characteristic polynomial $\chi_{T}(t)=(t+1)\left(t^{2}-2 t-1\right)$, so -1 is a simple eigenvalue of $T$, with corresponding eigenvector $v=\left[\begin{array}{lll}1 & -1 & 0\end{array}\right]$. Recall from $\S 6$ the dimension group representation $\delta: G \rightarrow \operatorname{aut}\left(\mathcal{G}_{T}, \mathcal{G}_{T}^{+}, \widehat{T}\right)$. Now $\mathbb{Z} v \subset \mathcal{G}_{T}$, and any element $\varphi \in \operatorname{aut}\left(\mathcal{G}_{T}, \mathcal{G}_{T}^{+}, \widehat{T}\right)$ must map $v$ to either $v$ or $-v$. Let $\theta(\varphi)$ be the choice 
of sign used, so $\theta: G \rightarrow\{ \pm 1\}$ with $\delta(\varphi)(v)=\theta(\varphi) v$. Since $\delta$ is a homomorphism, so is $\theta$.

We now show that $\theta$ has the required property. Fix $n \geq 1$. The blocks $M_{1}=c^{n} a$ and $M_{2}=c^{n} b$ have only trivial overlaps. Define $\varphi \in \operatorname{aut}\left(\sigma_{T}\right)$ to switch these blocks, and have no other effect. Clearly $\varphi_{n}$ fixes all points with period $\leq n$. We compute the action of $\delta\left(\varphi_{n}\right)$ on $v$. For $j=a, b, c$ choose the points $x^{j}$ so that

$$
x_{i}^{j}= \begin{cases}c & \text { if } i \neq 0, \\ j & \text { if } i=0 .\end{cases}
$$

Consider the 0-rays $C_{j}=x^{j}(-\infty, 0]^{*}$ for $j=a, b, c$. Under the correspondence of equivalence classes of beams to classes of eventually nonnegative vectors in $\mathcal{G}_{T}$ described in $\S 6$, each class $\left[C_{j}\right]$ corresponds to the class of the standard unit basis vector $e_{j}$. Now $\varphi_{n}$ exchanges the 0 -rays $C_{a}$ with $C_{b}$, and fixes $C_{c}$. Thus

$$
\delta\left(\varphi_{n}\right)=\left[\begin{array}{lll}
0 & 1 & 0 \\
1 & 0 & 0 \\
0 & 0 & 1
\end{array}\right],
$$

and $\delta\left(\varphi_{n}\right) v=-v$, so $\theta\left(\varphi_{n}\right)=-1$.

8. $p$-adic aspects of the gyration representation. Let us begin by recalling some notation from the previous section. We let $Q_{n}=Q_{n}\left(\sigma_{T}\right)$ denote the points in $X_{T}$ with least $\sigma_{T}$-period $n$, and $\mathcal{Q}_{n}=\mathcal{Q}_{n}\left(\sigma_{T}\right)=Q_{n} / \Sigma$ be the orbit space of $Q_{n}$ under $\sigma_{T}$. For each orbit $\gamma \in \mathcal{Q}_{n}$ pick $x_{\gamma} \in \gamma$. If $\varphi \in G$, then $\varphi x_{\gamma} \in \varphi(\gamma)$, so there is an integer $n(\gamma, \varphi)$ defined modulo $n$ so that $\varphi x_{\gamma}=\sigma^{n(\gamma, \varphi)} x_{\varphi \gamma}$. The $n$th gyration number of $\varphi$ is

$$
g\left(\varphi, \sigma_{T}\right)(n) \equiv \sum_{\gamma \in \mathcal{Q}_{n}\left(\sigma_{T}\right)} n(\gamma, \varphi) \in \mathbb{Z} / n \mathbb{Z}
$$

If $Q_{n}=\varnothing$, this number is defined by convention to be 0 . The gyration representation of $\varphi$ is the homomorphism sending $\varphi \in G$ to its gyration function

$$
g\left(\varphi, \sigma_{T}\right)=\left(g\left(\varphi, \sigma_{T}\right)(1), g\left(\varphi, \sigma_{T}\right)(2), \ldots\right) \in \prod_{n=1}^{\infty} \mathbb{Z} / n \mathbb{Z} .
$$

If $\sigma_{[r]}$ is the full $r$-shift, Boyle and Krieger [BK, Corollary 2.3] showed that $g\left(\sigma_{[r]}, \sigma_{[r]}\right)$ has infinite order in $\prod_{n=1}^{\infty} \mathbb{Z} / n \mathbb{Z}$. It follows that $\sigma_{[r]}$ is not a product of finite-order elements in $G$. Indeed, $\sigma_{[r]}$ is not even the limit in the periodic point topology of such products [BK, Theorem 2.8]. Also, $g\left(\sigma_{[r]} \times I, \sigma_{[r]} \times \sigma_{[s]}\right)$ has infinite order [BK, Proposition 2.4]. Using $p$-adic analysis, we shall extend these results to matrices $T$ for which the product $\operatorname{det}^{\times} T$ of the nonzero eigenvalues is not \pm 1 . The crucial fact is that, roughly speaking, for most primes $p$ the numbers $g\left(\sigma_{T}, \sigma_{T}\right)\left(p^{n}\right)$ converge $p$-adically to a transcendental limit in the $p$-adic completion $\mathbb{Q}_{p}$ of $\mathbb{Q}$.

To see the computational significance of this convergence, consider Table 1 of the gyration function of the 2-shift acting as a self-automorphism, evaluated at powers of 3 and of 5 . We have expanded the values to the appropriate base.

The evident stabilization of the digit coefficients for, say, $5^{n}$ can be expressed by saying that $g\left(\sigma_{[2]}, \sigma_{[2]}\right)\left(5^{n}\right)$ converges 5 -adically to some $\alpha \in \mathbb{Q}_{5}$. To prove this 


\begin{tabular}{|c|c|c|}
\hline$n$ & \multicolumn{1}{|c|}{$g\left(\sigma_{[2]}, \sigma_{[2]}\right)\left(3^{n}\right)$} & $g\left(\sigma_{[2]}, \sigma_{[2]}\right)\left(5^{n}\right)$ \\
\hline 1 & $2=2$ & $1=1$ \\
2 & $2=2+0 \cdot 3$ & $1=1+0 \cdot 5$ \\
3 & $11=2+0 \cdot 3+1 \cdot 3^{2}$ & $26=1+0 \cdot 5+1 \cdot 5^{2}$ \\
4 & $38=2+0 \cdot 3+1 \cdot 3^{2}+1 \cdot 3^{3}$ & $276=1+0 \cdot 5+1 \cdot 5^{2}+2 \cdot 5^{3}$ \\
5 & $38=2+0 \cdot 3+1 \cdot 3^{2}+1 \cdot 3^{3}$ & $2776=1+0 \cdot 5+1 \cdot 5^{2}+2 \cdot 5^{3}+4 \cdot 5^{4}$ \\
\hline
\end{tabular}

TABLE 1. Values of the gyration function for the 2-shift.

convergence, and identify the limit, note that since each orbit contributes 1 to the gyration function, we have

$$
\begin{aligned}
g\left(\sigma_{[2]}, \sigma_{[2]}\right)\left(5^{n}\right) & \equiv\left|\mathcal{Q}_{5^{n}}\left(\sigma_{[2]}\right)\right| \equiv \frac{\left|P_{5^{n}}\left(\sigma_{[2]}\right)\right|-\left|P_{5^{n-1}}\left(\sigma_{[2]}\right)\right|}{5^{n}} \\
& \equiv \frac{2^{5^{n}}-2^{5^{n-1}}}{5^{n}} \equiv\left(1-\frac{1}{5}\right) 2^{5^{n-1}}\left(\frac{2^{5^{n}-5^{n-1}}-1}{5^{n}-5^{n-1}}\right) .
\end{aligned}
$$

As $n \rightarrow \infty$, we have $2^{5^{n-1}} \rightarrow \omega(2) \in \mathbb{Q}_{5}$, a 4 th root of unity, the so-called Teichmüller representative of 2 . Also, since $5^{n}-5^{n-1} \rightarrow 0$ in $\mathbb{Q}_{5}$, the third factor converges to the 5 -adic derivative of $2^{x}$ at $x=0$, namely the 5 -adic logarithm $\log _{5} 2$ (see [Ko, Chapter 5, §1] for details). Thus $g\left(\sigma_{[2]}, \sigma_{[2]}\right)\left(5^{n}\right)$ converges 5-adically, with limit $\alpha=\left(1-\frac{1}{5}\right) \omega(2) \log _{5} 2$. If we denote $a_{0}+a_{1} 5+a_{2} 5^{2}+\cdots$ by.$a_{0} a_{1} a_{2} \ldots$, then $\omega(2)=.212134 \ldots, \frac{4}{5} \log _{5} 2=.330333 \ldots$, and the product is $\alpha=.101240 \ldots$, in agreement with the table entry. Furthermore, $\log _{5} 2$ is transcendental [Br]. Since $\frac{4}{5} \omega(2)$ is algebraic, the limit $\alpha$ is also transcendental. Since $\alpha \bmod 5^{n}$ is a unit in $\mathbb{Z} / 5^{n} \mathbb{Z}$, it follows that $g\left(\sigma_{[2]}, \sigma_{[2]}\right)\left(5^{n}\right)$ generates $\mathbb{Z} / 5^{n} \mathbb{Z}$ for $n \geq 1$. Hence $\sigma_{[2]}$ could not be a product of finite-order elements in $G$, for otherwise the orders of $g\left(\sigma_{[2]}, \sigma_{[2]}\right)(n)$ would be uniformly bounded in $n$. This analysis fails at the prime 2 , since $g\left(\sigma_{[2]}, \sigma_{[2]}\right)\left(2^{n}\right) \rightarrow 0$ in $\mathbb{Q}_{2}$. However, 2 is the only exceptional prime.

It is perhaps interesting to note that the $p$-adic convergence of the gyration function was first discovered by computer experimentation.

The ideas above extend to more general $\sigma_{T}$ by using the $p$-adic eigenvalues of $T$.

THEOREM 8.1. Suppose the product of the nonzero eigenvalues of $T$ is not \pm 1 . Then the gyration representation $g\left(\sigma_{T}, \sigma_{T}\right)$ has infinite order in $\prod_{n=1}^{\infty} \mathbb{Z} / n \mathbb{Z}$.

PROOF. Let the characteristic polynomial of $T$ be

$$
\chi_{T}(t)=t^{m}\left(t^{d}+a_{1} t^{d-1}+\cdots+a_{d}\right),
$$

where $a_{d} \neq \pm 1$ by assumption. Choose an odd prime $p$ relatively prime to $a_{d}$. A good account of the basic $p$-adic analysis used here is contained in [Ko]. Let $K$ be the splitting field of $\chi_{T}$ over the $p$-adic completion $\mathbb{Q}_{p}$ of $\mathbb{Q}$. The $p$-adic valuation $|\cdot|_{p}$ extends uniquely to $K$. Thus $\chi_{T}(t)$ factors over $K$ as $t^{m} \prod_{j=1}^{d}\left(t-\lambda_{j}\right)$. Since $a_{d}$ is a unit in the ring of integers in $K^{*}$, each $\left|\lambda_{j}\right|_{p}=1$. It follows there is an integer $r>0$ so that $\left|1-\lambda_{j}^{r}\right|_{p}<1$ for $1 \leq j \leq d$. We will prove that $g\left(\sigma_{T^{r}}, \sigma_{T^{r}}\right)\left(p^{n}\right)$ 
converges $p$-adically to a nonzero limit, show this forces $g\left(\sigma_{T^{r}}, \sigma_{T^{r}}\right)$ to have infinite order, and conclude from this that $g\left(\sigma_{T}, \sigma_{T}\right)=g\left(\sigma_{T}\right)$ also has infinite order.

For arbitrary $U$, we have $\left|Q_{n}\left(\sigma_{U}\right)\right|=\sum_{d \mid n} \mu(n / d) \operatorname{tr} U^{d}$, where $\mu$ is the Möbius function, and $g\left(\sigma_{U}, \sigma_{U}\right)(n)=\left|Q_{n}\left(\sigma_{U}\right)\right| / n$. Hence

$$
\begin{aligned}
g\left(\sigma_{T^{r}}, \sigma_{T^{r}}\right)\left(p^{n}\right) & =\frac{1}{p^{n}}\left\{\operatorname{tr}\left(T^{r}\right)^{p^{n}}-\operatorname{tr}\left(T^{r}\right)^{p^{n-1}}\right\} \\
& =\left(1-\frac{1}{p}\right) \sum_{j=1}^{d} \lambda_{j}^{r p^{n-1}}\left(\frac{\lambda_{j}^{r\left(p^{n}-p^{n-1}\right)}-1}{p^{n}-p^{n-1}}\right) .
\end{aligned}
$$

Since $\left|1-\lambda_{j}^{r}\right|_{p}<1$, it follows that $\lambda_{j}^{r p^{n}} \rightarrow 1$ in $K$. The second factor in the sum converges to the $p$-adic derivative of $\lambda_{j}^{r x}$ with respect to $x$ at $x=0$, which is $r \log _{p} \lambda_{j}$. Here $\log _{p} y$ is the $p$-adic logarithm defined for $y \in K$ with $|y-1|_{p}<1$ by the convergent series $\log _{p} y=\sum_{n=1}^{\infty}(-1)^{n+1}(y-1)^{n} / n$. Thus as $n \rightarrow \infty$,

$$
g\left(\sigma_{T^{r}}, \sigma_{T^{r}}\right)\left(p^{n}\right) \rightarrow\left(1-\frac{1}{p}\right) \log _{p} a_{d}^{r} \neq 0,
$$

the nonvanishing following since $a_{d} \neq \pm 1$. This means that as $n$ increases, the $p$ adic expansion of $g\left(\sigma_{T^{r}}, \sigma_{T^{r}}\right)\left(p^{n}\right)$ has low order coefficients that stabilize to nonzero values. In particular, if

$$
\left|\left(1-\frac{1}{p}\right) \log _{p} a_{d}^{r}\right|_{p}>p^{-k},
$$

then $p^{n-k} g\left(\sigma_{T^{r}}, \sigma_{T^{r}}\right)\left(p^{n}\right) \not \equiv 0 \bmod p^{n}$ for $n$ sufficiently large. This clearly implies that $g\left(\sigma_{T^{r}}, \sigma_{T^{r}}\right)$ has infinite order. We conclude by showing this forces $g\left(\sigma_{T}, \sigma_{T}\right)$ to also have infinite order. First note that $g\left(\sigma_{T^{r}}, \sigma_{T^{r}}\right)=g\left(\sigma_{T}^{r}, \sigma_{T}^{r}\right)=r g\left(\sigma_{T}, \sigma_{T}^{r}\right)$. The calculation of Proposition 1.6 of $[\mathbf{B K}]$ shows that if $a_{j}=a_{j}(r, n) \in \mathbb{Z}$ is defined so $r a_{j} \equiv j \bmod j n$ when $j=(r, j n)$ and 0 otherwise, then

$$
g\left(\varphi, \sigma_{T}^{r}\right)(n) \equiv \sum_{j \mid r} a_{j} g\left(\varphi, \sigma_{T}\right)(j n) \bmod n
$$

Apply this to $\varphi=\sigma_{T}$ in the above to express $g\left(\sigma_{T^{r}}, \sigma_{T^{r}}\right)(n)$ as an integral combination of the $g\left(\sigma_{T}, \sigma_{T}\right)(j n)$ for $j \mid r$. Thus if $g\left(\sigma_{T}, \sigma_{T}\right)$ had finite order, so would $g\left(\sigma_{T^{r}}, \sigma_{T^{r}}\right)$. This contradiction establishes the theorem.

REMARKS. 1. If $\lambda$ is a unit in $K$, the splitting field of $\chi_{T}$ over $\mathbb{Q}_{p}$, then $\lambda^{p^{n}}$ converges to a $\left(p^{e}-1\right)$ st root of unity $\omega(\lambda)$ in $K$, where $e$ is the ramification index of $K$ over $\mathbb{Q}_{p}$. The above shows that we always have in $\mathbb{Q}_{p}$ that

$$
\lim _{n \rightarrow \infty} g\left(\sigma_{T}, \sigma_{T}\right)\left(p^{n}\right)=\left(1-\frac{1}{p}\right) \log _{p}\left(\prod_{j=1}^{d} \lambda_{j}^{\omega\left(\lambda_{j}\right)}\right) .
$$

However, we cannot conclude from this that the limit is nonzero. By taking $r$ th powers, we force $\omega\left(\lambda_{j}^{r}\right)=1$, so the product in (8-1) is $a_{d}^{r} \neq 1$, and the limit does not vanish. The general question of whether (8-1) is nonzero hinges on how the $\lambda_{j}$ and the corresponding root of unity $\omega\left(\lambda_{j}\right)$ interact. For example, if the $\lambda_{j}$ are multiplicatively independent, then the $p$-adic versions of Baker's transcendence results $[\mathbf{B r}]$ show that $(8-1)$ is not zero. 
2. If the product of the nonzero eigenvalues of $T$ is \pm 1 , all that is needed to prove the theorem is to find one prime $p$ for which (8-1) does not vanish. Since this is the "typical" case, finding such a $p$ can usually be done by hand. For example, if $T=\left[\begin{array}{ll}1 & 1 \\ 1 & 0\end{array}\right]$ we can take $p=3$ and simply check that the product in (8-1) is not \pm 1 . Indeed, all but finitely many primes will work in this case.

3 . If $\operatorname{det}^{\times} T \neq \pm 1$, the roots of unity $\omega\left(\lambda_{j}\right)$ in (8-1) are merely a nuisance, to be eliminated by passing to $r$ th powers. However, when $\operatorname{det}^{\times} T= \pm 1$ it is essential that at least some of the $\omega\left(\lambda_{j}\right)$ not be 1 to obtain a nonzero limit in (8-1).

The proof of Theorem 8.1 can be modified to obtain the following.

THEOREM 8.2. Suppose the product of the nonzero eigenvalues of $T$ is not \pm 1 . Then $g\left(\sigma_{T} \times I, \sigma_{T} \times \sigma_{U}\right)$ has infinite order.

Proof. Choose a prime $p$ relatively prime to $\operatorname{det}^{\times} T$ and to $\operatorname{det}^{\times} U$. We first compute the gyration function $g\left(\sigma_{T} \times I, \sigma_{T} \times \sigma_{U}\right)\left(p^{n}\right)$. Now

$$
Q_{p^{n}}\left(\sigma_{T} \times \sigma_{U}\right)=\bigcup_{k=0}^{n}\left\{Q_{p^{n}}\left(\sigma_{T}\right) \times Q_{p^{k}}\left(\sigma_{U}\right) \cup Q_{p^{k}}\left(\sigma_{T}\right) \times Q_{p^{n}}\left(\sigma_{U}\right)\right\} .
$$

If $(x, y) \in Q_{p^{k}}\left(\sigma_{T}\right) \times Q_{p^{n}}\left(\sigma_{U}\right)$, then the smallest $q \geq 0$ for which $\left(\sigma_{T} \times I\right)^{q}(x, y)$ is in the $\sigma_{T} \times \sigma_{U}$-orbit of $(x, y)$ is $q=p^{k}$, and this element is $(x, y)$ itself. It follows that such orbits contribute $0 \bmod p^{n}$ to the gyration function. Let $0 \leq k \leq n-1$. For each orbit $\gamma \in \mathcal{Q}_{p^{n}}\left(\sigma_{T}\right)$ pick $x_{\gamma} \in \gamma$. Then

$$
\bigcup_{\gamma \in \mathcal{Q}_{p^{n}}\left(\sigma_{T}\right)}\left\{x_{\gamma}\right\} \times Q_{p^{k}}\left(\sigma_{U}\right)
$$

is a complete set of orbit representatives for $Q_{p^{n}}\left(\sigma_{T}\right) \times Q_{p^{k}}\left(\sigma_{U}\right)$. Since

$$
\left(\sigma_{T} \times I\right)(x, y)=\left(\sigma_{T} \times \sigma_{U}\right)\left(x, \sigma_{U}^{-1} y\right),
$$

each representative contributes 1 to the gyration function. Hence

$$
\begin{aligned}
g\left(\sigma_{T} \times I, \sigma_{T} \times \sigma_{U}\right)\left(p^{n}\right) & \equiv\left|\mathcal{Q}_{p^{n}}\left(\sigma_{T}\right)\right| \sum_{k=0}^{n-1}\left|Q_{p^{k}}\left(\sigma_{T}\right)\right| \\
& \equiv \frac{1}{p^{n}}\left(\operatorname{tr} T^{p^{n}}-\operatorname{tr} T^{p^{n-1}}\right)\left(\operatorname{tr} U^{p^{n-1}}\right) .
\end{aligned}
$$

Suppose $K$ is a splitting field for $\chi_{T}(t)$ and $\chi_{U}(t)$ over $\mathbb{Q}_{p}$. Let $T$ have nonzero $p$-adic eigenvalues $\lambda_{1}, \ldots, \lambda_{d}$, and let those of $U$ be $\mu_{1}, \ldots, \mu_{e}$. Then by the above

$$
g\left(\sigma_{T} \times I, \sigma_{T} \times \sigma_{U}\right)\left(p^{n}\right) \equiv \sum_{i, j}\left(\frac{\lambda_{i}^{p^{n}}-\lambda_{i}^{p^{n-1}}}{p^{n}}\right) \mu_{j}^{p^{n-1}} .
$$

The $p$-adic convergence of this expression follows as before. To obtain a nonzero limit, pass to $r$ th powers to obtain $\lambda_{i}^{r p^{n}} \rightarrow 1, \mu_{j}^{r p^{n}} \rightarrow 1$, and argue as in the proof of Theorem 8.1.

Theorem 2.9 of $[\mathbf{B K}]$ implies that if $\operatorname{det}^{\times} T \neq \pm 1$, then $\sigma_{T}$ is not the product of finite-order elements in $G$. This is also a consequence of Theorem 8.1. The following, which generalizes Theorem 2.8 of $[\mathbf{B K}]$, shows $\sigma_{T}$ is not even a limit of such products. 
PROPOSITION 8.3. Suppose the product of the nonzero eigenvalues of $\sigma_{T}$ is not \pm 1 . Then $\sigma_{T}$ is not the limit, in the periodic point topology on $G$, of products of finite-order elements.

PROOF. The periodic point topology was defined in $\S 7$ to be the coarsest making the restriction maps $G \rightarrow \operatorname{aut}\left(Q_{n}, \sigma_{T}\right)$ continuous. From the proof of Theorem 8.1 we can conclude there is a prime $p$ and an integer $m$ so that for all $n \geq m$ we have

$$
g\left(\sigma_{T}, \sigma_{T}\right)\left(p^{n}\right) \not \equiv 0 \bmod p^{m} .
$$

The result now follows exactly as in the proof of Theorem 2.8 of [BK] by using Proposition 2.7 there.

REMARK 4. All of the above results will hold even if $\operatorname{det}^{\times} T= \pm 1$, provided the prime $p$ used in the proofs can be chosen so that (8-1) is nonzero. As indicated in Remark 2, in specific case this can usually be done ad hoc.

Problem 8.4. Remove the restriction $\operatorname{det}^{\times} T \neq \pm 1$ from the above.

The proof of Theorem 8.1 shows that $g\left(\sigma_{T}, \sigma_{T}\right)\left(p^{n}\right)$ converges $p$-adically for all primes $p$. It is also easy to see that if $\varphi$ has finite order, then $g\left(\varphi, \sigma_{T}\right)\left(p^{n}\right) \rightarrow 0$ in $\mathbb{Q}_{p}$. Thus for automorphisms $\varphi$ constructed by known methods, $g\left(\varphi, \sigma_{T}\right)\left(p^{n}\right)$ always converges $p$-adically.

QUESTION 8.5. Does the gyration function $g\left(\varphi, \sigma_{T}\right)\left(p^{n}\right)$ converge $p$-adically for every $\varphi \in \operatorname{aut}\left(\sigma_{T}\right)$ ?

9. Compact invariant sets. In $\S 7$ we investigated $G=\operatorname{aut}\left(\sigma_{T}\right)$ by studying its action on the compact $G$-invariant sets $Q_{n}\left(\sigma_{T}\right)$ of points with least $\sigma_{T}$-period $n$. Are there other compact $G$-invariant subsets of $X_{T}$ ? In this section we show that, modulo possibly a few orbits of low period, every compact $G$-invariant subset is a finite union of various $Q_{n}\left(\sigma_{T}\right)$ or all of $X_{T}$. We also discuss the implications for the action of $G$ on the compact space of subshifts equipped with the Hausdorff metric.

Several constructions and proofs to follow are made clearer by the process of "passing to a higher order block presentation", described as follows. Recall from $\S 1$ that if $T$ is $r \times r$, then the graph of $T$ has $r$ states or nodes, and $\sum_{i, j=1}^{r} T_{i j}$ symbols or edges. Order the set $\mathcal{L}$ of symbols arbitrarily. Let $E(T)$ be the transition matrix for symbols, defined by $E(T)_{x y}=1$ if the terminal state of $x$ matches the initial state of $y$, and 0 otherwise. Iterating this procedure yields the transition matrix $E^{n}(T)=E\left(E^{n-1}(T)\right)$ for allowed $n$-blocks of symbols. Specifically, we define the $n$-block presentation of $T$ to be $T^{[n]}=E^{n-1}(T)$, where $E^{0}(T)=T$. The symbols for $T^{[n]}$ are then the allowed $n$-blocks of $\sigma_{T}$, i.e. $\mathcal{L}_{T^{[n]}}=B_{n}\left(\sigma_{T}\right)$. If $B, C \in \mathcal{L}_{T^{[n]}}$, then $T_{B C}^{[n]}=1$ if $B[1, n-1]=C[0, n-2]$, and 0 otherwise. Thus the symbols in a point $x \in X_{T}$ have simply been recoded in $X_{T^{[n]}}$ by using $n$-blocks of symbols.

There is a natural conjugacy $\beta_{n}: X_{T} \rightarrow X_{T^{[n]}}$ defined by

$$
\left(\beta_{n} x\right)_{i}=x[i-k, i-k+n-1], \quad \text { where } k=\left[\frac{n}{2}\right] .
$$

The metric on $X_{T^{(n)}}$ is understood to be that induced from $X_{T}$ under $\beta_{n}$. With this convention, the maximum of the diameters of the sets $B^{*}=\left\{y \in X_{T^{(n)}}: y_{0}=B\right\}$ over $B \in \mathcal{L}_{T^{[n]}}$ tends to 0 as $n \rightarrow \infty$. 
If $K$ is a subshift of $X_{T}$, let $B(K)$ be the collection of all blocks that occur in some point in $K$. For $x \in X_{T}$, put $B(x)=B(\overline{\Sigma x})$. If $B \in B_{n}\left(X_{T}\right)$, put $B^{*}=$ $\left\{x \in X_{T}: x[0, n-1]=B\right\}$. Here the reader should distinguish $B$ considered as an $n$-block from $X_{T}$ from $B$ considered as a symbol from $X_{\left.T^{[n]}\right]}$. The homeomorphism $\beta_{n}$ introduces a factor of $\sigma_{T}^{[n / 2]}$ between the two meanings of $B^{*}$. In particular,

$$
\max \left\{\operatorname{diam}\left(B^{*}\right): B \in \mathcal{L}_{T^{[n]}}\right\} \rightarrow 0 \quad \text { as } n \rightarrow \infty .
$$

Thus if $Y_{1}, \ldots, Y_{k}$ are disjoint subshifts of $X_{T}$, for all large enough $n$ the sets $\mathcal{L}_{T^{[n]}}\left(Y_{j}\right)$ of symbols from $\mathcal{L}_{T^{[n]}}$ occurring in $Y_{j}$ are disjoint. Furthermore, if $Z \subset$ $X_{T}$ is a shift of finite type, for all large enough $n$ we can represent $Z$ as 1-step on the symbols $\mathcal{L}_{T^{[n]}}(Z)$.

For completeness, we prove a standard technical result. A stronger version is contained in [DGS, 26.17].

LemMA 9.1. Suppose $B \in B\left(X_{T}\right)$ and that $B \notin B(x)$. Then there is an infinite mixing shift of finite type $Z \subset X_{T}$ with $B \in B(Z)$ and $Z$ disjoint from $\overline{\Sigma x}$.

PROOF. Since our standing assumption is that $\sigma_{T}$ is mixing, there are $C_{j} \in$ $B\left(X_{T}\right), j=0,1$, with relatively prime lengths, with $B C_{j} B \in B\left(X_{T}\right)$, and such that $B$ occurs only as the initial and terminal block in each $B C_{j} B$. Form the shift of finite type $Z \subset X_{T}$ of points of the form $\cdots B C_{i_{-1}} B C_{i_{0}} B C_{i_{1}} B \cdots$ with $i_{k}=0$ or 1 for $k \in \mathbb{Z}$. Since the $C_{j}$ have relatively prime lengths, $\left.\sigma_{T}\right|_{Z}$ is mixing with positive entropy. Let $n \geq 2\left(\left|C_{0}\right|+\left|C_{1}\right|+|B|\right)$. Every block in $B_{n}(Z)$ contains $B$ as a subblock, while $B$ does not occur in $x$. Thus, by passing to a higher order block presentation, for large enough $n$, we can realize $Z$ as a 1-step shift of finite type on an alphabet disjoint from the symbols occurring in $x$. Thus $Z \cap \overline{\Sigma x}=\varnothing$.

THEOREM 9.2. The automorphism group orbit $G x$ of $x \in X_{T}$ is dense if and only if $x$ is not $\sigma_{T}$-periodic.

PROOF. Since the period of a point is preserved under an automorphism, a $\sigma_{T}$-periodic point $x$ clearly has $G x$ finite.

Now assume $x$ is not $\sigma_{T}$-periodic. To show $\overline{G x}=X_{T}$, it is enough to prove that for every $B \in B\left(X_{T}\right)$ there is a $\varphi \in G$ with $B \in B(\varphi x)$. If $B \in B(x)$, take $\varphi=I$. Thus we assume $B \notin B(x)$, and find $\varphi$. By Lemma 9.1, there is a mixing shift of finite type $Z$ disjoint from $\overline{\Sigma x}$ with $B \in B(Z)$.

We first treat the case that a block in $B(x)$ occurs exactly once in $x$. By passing to the $n$-block presentation of $X_{T}$, we can assume $Z$ is a 1-step shift of finite type on an alphabet $\mathcal{L}(Z) \subset \mathcal{L}_{T[n]}=B_{n}\left(\sigma_{T}\right)$ disjoint from the symbols occurring in $x$, that $B$ occurs as a subword of $b \in \mathcal{L}(Z)$, and that a symbol $a \in \mathcal{L}_{T}^{[n]}$ occurs in $x$ at a unique index, which by applying a power of $\sigma_{T^{[n]}}$ we can assume is 0 . For the rest of this case, all symbols and blocks are from the $n$-block presentation. Let $l$ be a transition length for $X_{T[n]}$ and for $Z$. Since both are mixing, there is a block $E=a A b C b A^{\prime} x[4 l, 6 l]$ such that $|A|<l,\left|A^{\prime}\right|<l, a$ does not occur in $A$ and occurs in $A^{\prime}$ at most once, $C \in B(Z)$, and $|E|=6 l+1$. We claim that $E$ and $F=x[0,6 l]$ overlap only trivially in the sense of $\S 2$. Since $a$ occurs only as the initial symbol in $F$ while $E$ contains symbols from $Z$ never used in $F$, it follows $E$ does not overlap $F$, and that $F$ only overlaps itself trivially. The only nontrivial overlap of $E$ with itself could occur with the occurrence of $a$ in $A^{\prime}$, but this is ruled 
out since this would force $x[4 l, 6 l]$ and $C$ to have symbols in common. Thus, as in $\S 2$, the map $\varphi \in G$ defined by interchanging $E$ and $F$ and having no other effect is a well-defined involution. Since the symbol $b$ from the $n$-block presentation occurs in $\varphi x$, it follows that the original block $B$ occurs in $\varphi x$, completing this case.

We next turn to the recurrent case. Here blocks in $x$ can recur with distressing frequency, making the nonoverlapping condition for markers more difficult to achieve. The reader should keep in mind the Thue-Morse sequence, where every allowed block of length $n$ recurs within $8 n$, to better understand the complications below.

Let $x, B$, and $Z$ retain their meanings, and assume we have already passed to a higher order block presentation with $B$ a subword of $b \in \mathcal{L}(Z)$ and $\mathcal{L}(Z) \cap \mathcal{L}(x)=\varnothing$. Choose symbols $a, c \in \mathcal{L}(x)=B_{1}(x)$ so that there are words $a A b, b A^{\prime} c \in B\left(X_{T}\right)$, where $A$ and $A^{\prime}$ use no symbols from $\mathcal{L}(x)$, and so that some word $a F c$ occurs in $x$. We claim we can assume there are arbitrarily long words of the form $a F c$ in $B(x)$. For if not, the longest such word could occur only once, and the previous case applies. Let $l$ be a transition length for $X_{T}$ and for $Z$. Adjusting $x$ by a power of $\sigma_{T}$ if necessary, there is a $k \geq 4 l$ with $x_{0}=a$ and $x_{k}=c$, and a word $C \in B(Z)$ so that $D=a A b C b A^{\prime} c \in B(X)$ with $|D|=k+1$. Note that the only symbols in $D$ that are also in $\mathcal{L}(x)$ are the initial and terminal symbols. Let $E=x[0, k]$. Since $x$ is not $\sigma_{T}$-periodic, there is a $p>k$ so that $x[-p, p-j] \neq x[-p+j, p]$ for $0<j<k$. Define $\xi: B_{2 p+1}(X) \rightarrow B_{2 p+1}(X)$ by replacing each occurrence of $D$ by $E$; if a block begins with a terminal segment of $D$, replace it with the corresponding terminal segment of $E$, and similarly at the other end. Since $D$ cannot overlap itself except possibly in one symbol, $\xi$ is well-defined. Note that $\xi(x[-p, p])=x[-p, p]$.

Let $\mathcal{M}=\xi^{-1}(x[-p, p]) \cap B_{p}(X)\{D, E\} B_{p-k}(X)$. Then $\mathcal{M}$ has the nonoverlapping property that if $M, M^{\prime} \in \mathcal{M}$, then $M[-p, p-j] \neq M^{\prime}[-p+j, p]$ for $0<j<k$, since otherwise applying $\xi$ would contradict the choice of $p$. Define $\varphi \in G$ as follows. If $y[-p, p] \in \mathcal{M}$, then put

$$
(\varphi y)[0, k]= \begin{cases}E & \text { if } y[0, k]=D \\ D & \text { if } y[0, k]=E\end{cases}
$$

Declare $\varphi$ to have no other action. Because of the nonoverlapping property of the words in $\mathcal{M}$ mentioned above, a symbol in $y$ can be affected at most once by $\varphi$, so $\varphi$ is well-defined. If $y[-p, p] \in \mathcal{M}$, then $(\varphi y)[-p, p] \in \mathcal{M}$, proving that $\varphi$ is an involution. Finally, since the symbol $b$ from the higher order presentation occurs in $D$, which is a subblock of $(\varphi x)[-p, p]$, we see that the original block $B \in B(x)$, completing the proof.

THEOREM 9.3. Let $\sigma_{T}$ be a mixing shift of finite type, and define $n_{0}(T)$ as in Theorem 7.2. If $K$ is a compact $G$-invariant set, then either $K=X_{T}$, or $K$ is a finite union of $Q_{n_{j}}\left(\sigma_{T}\right)$ with $n_{j} \geq n_{0}(T)$ together with possibly a finite number of orbits with periods $<n_{0}(T)$.

PROOF. If $K$ contains a nonperiodic point, then $K=X_{T}$ by Theorem 9.2. If $x \in K \cap Q_{n}\left(\sigma_{T}\right)$ for some $n \geq n_{0}(T)$, then by Proposition 7.3 we have $Q_{n}\left(\sigma_{T}\right) \subset K$. If $n_{j} \rightarrow \infty$, then $\bigcup_{j=1}^{\infty} Q_{n_{j}}\left(\sigma_{T}\right)$ is dense, so either $K$ contains only a finite number of the sets $Q_{n}\left(\sigma_{T}\right)$ for $n \geq n_{0}(T)$, or $K=X_{T}$. 
REMARKS 1. Until the question of switching points with small periods, such as in Example 7.3, is settled, the possibility remains that for some $n<n_{0}(T)$ the set $Q_{n}\left(\sigma_{T}\right)$ could have proper $G$-invariant subsets.

2. An irreducible shift of finite type is a finite tower over a mixing base shift of finite type, so Theorem 9.3 easily extends to this case.

Let $S$ denote the compact space of subshifts of $\left(X_{T}, \sigma_{T}\right)$ equipped with the Hausdorff metric. In this context, two subshifts $Y, Z \in S$ are close if for a large $n$ the sets $B_{n}(Y)$ and $B_{n}(Z)$ coincide. This is the topological analogue of the weak topology on $\sigma_{T}$-invariant measures used in the next section. From this description it is evident that $G$ acts continuously on $S$. Theorem 9.3 identifies all but a finite number of the fixed points of this action. The result also shows that if $Y \in S$ is infinite, then $X_{T}$ is in the $G$-orbit closure of $Y$ in $S$. We now generalize this argument.

PROPOSITION 9.4. If $Y \subset Z \subset X_{T}$ are infinite subshifts with $Z$ mixing, then $Z$ is in the $G$-orbit closure of $Y$ in the Hausdorff metric.

ProOF. Fix an $n \geq 1$, and enumerate the blocks of $B_{n}(Z)$ as $B_{1}, \ldots, B_{k}$. Since $\sigma_{T} \mid Z$ is mixing, there is $B \in B(Z)$ which contains each $B_{j}$. Pick $y \in Y$ with infinite orbit. Let $Z^{\prime} \subset X_{T}$ be the shift of finite type so that $B_{n}\left(Z^{\prime}\right)=B_{n}(Z)$. Then $Z^{\prime}$ is mixing since $Z$ is. The construction of $\varphi$ with $B \in B(\varphi y)$ in the proof of Theorem 9.2 can be carried out with $Z^{\prime}$ as the ambient space, so $\varphi Y \subset Z^{\prime}$. Since each $B_{j}$ occurs in $B$, we can conclude that $B_{n}(\varphi Y)=B_{n}\left(Z^{\prime}\right)=B_{n}(Z)$. Thus every neighborhood of $Z$ in $S$ contains an image $\varphi Y$ for some $\varphi \in G$, completing the proof.

It is more difficult to determine when a subshift $Z$ in general position is in the $G$-orbit closure of $Y$. Two necessary conditions follow.

PROPOSITION 9.5. If $Y$ and $Z$ are subshifts of $X_{T}$ and $Z$ is in the $G$-orbit closure of $Y$, then

(1) $h\left(\left.\sigma_{T}\right|_{Z}\right) \geq h\left(\left.\sigma_{T}\right|_{Y}\right)$,

(2) $\left|Q_{n}\left(\sigma_{T} \mid z\right)\right| \geq\left|Q_{n}\left(\sigma_{T} \mid Y\right)\right|$ for $n \geq 1$.

ProOF. Suppose $\varphi_{k} \in G$ with $\varphi_{k} Y \rightarrow Z$. Since topological entropy is upper semicontinuous on $S$, and $h\left(\left.\sigma_{T}\right|_{\varphi_{k} Y}\right)=h\left(\left.\sigma_{T}\right|_{Y}\right)$, property (1) holds. Also, for fixed $n$ we must have for large enough $k$ that $\varphi_{k}\left[Q_{n}\left(\left.\sigma_{T}\right|_{Y}\right)\right] \subset Q_{n}\left(\sigma_{T} \mid z\right)$, from which (2) follows.

PROBLEM 9.6. Determine necessary and sufficient conditions on $Y, Z \in S$ for $Z$ to be in the $G$-orbit closure of $Y$.

Of course, Example 7.3 shows that this problem is not solved even for subshifts with a finite number of points.

10. Orbits of measures. Let $P\left(X_{T}\right)$ denote the compact convex set of $\sigma_{T^{-}}$ invariant probability measures on $X_{T}$, and $M\left(X_{T}\right)$ be the $\sigma_{T}$-invariant nonnegative measures, each equipped with the weak topology. The group $G=\operatorname{aut}\left(\sigma_{T}\right)$ acts naturally on both spaces, and the unique measure $\mu_{T}$ of maximal entropy is invariant under every $\varphi \in G[\mathbf{C P}]$. Are there other continuous $G$-invariant measures? We show there are none, and obtain a complete characterization of the $G$-orbit closure in $P\left(X_{T}\right)$ of a continuous probability measure. 
To state our result, let $h(\mu)$ denote the entropy of $\sigma_{T}$ with respect to $\mu \in M\left(X_{T}\right)$. If $P_{0}$ is the partition of $X_{T}$ by the 0 th coordinate, then

$$
h(\mu)=\lim _{n \rightarrow \infty} \frac{1}{n} \sum_{A \in \bigvee_{j=0}^{n-1} \sigma_{T}^{-j} p_{0}}-\mu(A) \log \mu(A) .
$$

Observe that this formula applies even to nonnegative measures with total mass different from 1 , and that if $r, s \geq 0$ and $\mu, \nu \in M\left(X_{T}\right)$, then [W, Theorem 8.1] $h(r \mu+s \nu)=r h(\mu)+s h(\nu)$. We shall use a version of the ergodic decomposition suited to our needs. For $\mu \in P\left(X_{T}\right)$ there is a measurable function $t \mapsto \mu^{t}$ from $[0,1]$ to $P\left(X_{T}\right)$ so that each $\mu^{t}$ is ergodic under $\sigma_{T}$, if $E \subset X_{T}$ is Borel then $\mu^{t}(E)$ is measurable in $t$ with $\mu(E)=\int_{0}^{1} \mu^{t}(E) d t$, and $h\left(\mu^{t}\right)$ is decreasing in $t$. Note that the $\mu^{t}$ need not be distinct. Indeed, if $\mu$ is already ergodic, then $\mu^{t}=\mu$ for a.e. $t$. All but the entropy statement follows from the standard ergodic decomposition [DGS, Chapter 13]; $h\left(\mu^{t}\right)$ can be arranged to be decreasing by a measurable rearrangement of $[0,1]$. A limiting form of the linearity of $h$ mentioned above shows that if $E \subset$ $[0,1]$, then $h\left(\int_{E} \mu^{t} d t\right)=\int_{E} h\left(\mu^{t}\right) d t$.

Define the cumulative entropy $H_{\mu}(t)=\int_{0}^{t} h\left(\mu^{s}\right) d s$ for $0 \leq t \leq 1$. This function can be defined without reference to the ergodic decomposition by

$$
H_{\mu}(t)=\sup \left\{h(\nu): \nu \in M\left(X_{T}\right), \nu \leq \mu, \nu\left(X_{T}\right)=t\right\} .
$$

For by ergodicity of the $\mu^{t}$, if $\nu \leq \mu$ with $\nu\left(X_{T}\right)=t$, then $\nu=\int_{0}^{1} \rho(s) \mu^{s} d s$ with $\int_{0}^{1} \rho(s) d s=t$. Thus $h(\nu)=\int_{0}^{1} \rho(s) h\left(\mu^{s}\right) d s$ which, by monotonicity of $h\left(\mu^{t}\right)$, has maximum value $H_{\mu}(t)$ when $\rho$ is the indicator function of $[0, t]$. Our characterization of orbit measures is the following.

THEOREM 10.1. Let $\sigma_{T}$ be a mixing shift of finite type on $X_{T}$, let $G=\operatorname{aut}\left(\sigma_{T}\right)$, and suppose $\mu \in P\left(X_{T}\right)$ is continuous. Then $\nu$ is in the $G$-orbit closure of $\mu$ iff $H_{\mu} \leq H_{\nu}$ on $[0,1]$.

COROLlaRY 10.2. The measure $\mu_{T}$ of maximal entropy for $\sigma_{T}$ is the only $G$-invariant continuous probability measure on $X_{T}$.

Proof of THE Corollary. Suppose $\mu \in P\left(X_{T}\right)$ is continuous and $G$ invariant. Then $h\left(\mu^{t}\right) \leq h\left(\mu_{T}^{t}\right)=h\left(\sigma_{T}\right)$ for all $t$, so $H_{\mu} \leq H_{\mu_{T}}$. Thus $\mu_{T} \in$ $\overline{G \mu}=\{\mu\}$.

Note that if $\mu$ and $\nu$ are ergodic, the condition of Theorem 10.1 reduces to $h(\mu) \leq h(\nu)$.

We will first prove the necessity of the condition $H_{\mu} \leq H_{\nu}$ by using upper semicontinuity of entropy. The main work is to prove its sufficiency. We first find in Lemma 10.3 a partition $0=t_{0}<t_{1}<\cdots<t_{K}=1$ so that if $J_{k}=\left[t_{k-1}, t_{k}\right)$, $\alpha_{k}=\left|J_{k}\right|$, and $\mu_{k}=\alpha_{k}^{-1} \int_{J_{k}} \mu^{t} d t$, then $\mu$ is a convex combination $\sum_{k=1}^{K} \alpha_{k} \mu_{k}$, where the $\mu_{k}$ are mutually singular and $h\left(\mu_{k}^{t}\right)$ is almost constant. We then use the Convex Approximation Lemma 10.4 to perturb $\nu$ to $\tilde{\nu}=\sum_{k=1}^{K} \alpha_{k} \tilde{\nu}_{k}$, so that the $\tilde{\nu}_{k}$ are mixing Markov measures with disjoint supports and $h\left(\tilde{\nu}_{k}\right)>h\left(\mu_{k}\right)$. Using a Rohlin stack argument in Lemma 10.5 based on a marker, and assuming that almost every $\mu^{t}$ has full support, we construct a marker automorphism in Lemma 10.6 mapping $\mu$ close to $\nu$ that does not affect a preassigned subshift. The case when 
$\mu$ does not have full support, involving difficulties similar to those in the second half of the proof of Theorem 9.2, is treated in Lemma 10.7. Using a decomposition from Lemma 10.8, the pieces of the proof for sufficiency are, at last, assembled.

ProOF OF THEOREM 10.1 (NECESSITY). Suppose $\mu \in P\left(X_{T}\right)$ is continuous with ergodic decomposition $\mu=\int_{0}^{1} \mu^{s} d s$, and $\varphi_{n} \in G$ has $\varphi_{n} \mu \rightarrow \nu$ weakly. Fix $t \in[0,1]$ and put $\mu^{\prime}=\int_{0}^{t} \mu^{s} d s$. By compactness, a subsequence of $\varphi_{n} \mu^{\prime}$ converges to some $\nu^{\prime}$, where $\nu^{\prime}\left(X_{T}\right)=t$ and $\nu^{\prime} \leq \nu$. By the intrinsic definition of $H_{\nu}$ given above, we get $h\left(\nu^{\prime}\right) \leq H_{\nu}(t)$. Using the upper semicontinuity of entropy [W, Theorem 8.2] and that $h\left(\mu^{\prime}\right)=h\left(\varphi_{n} \mu^{\prime}\right)$ for all $n$, we have

$$
H_{\mu}(t)=h\left(\mu^{\prime}\right)=\limsup _{n \rightarrow \infty} h\left(\varphi_{n} \mu^{\prime}\right) \leq h\left(\nu^{\prime}\right) \leq H_{\nu}(t) .
$$

We begin building the machinery to prove sufficiency. For $\mu \in M\left(X_{T}\right)$ let $\operatorname{supp}(\mu)$ denote the complement of the largest open $\mu$-null set.

LEMMA 10.3. Let $\mu, \nu \in P\left(X_{T}\right)$, let $Z \subset X_{T}$ be a proper subshift, and suppose $h(\nu)>h(\mu)$. For every neighborhood $V$ of $\nu$ in $P\left(X_{T}\right)$ and $\varepsilon>0$, there is a mixing Markov measure $\tilde{\nu} \in V$ with $|h(\tilde{\nu})-h(\nu)|<\varepsilon, \operatorname{supp}(\tilde{\nu}) \cap Z=\varnothing$, and $\tilde{\nu} \perp \mu$.

Proof. Let $V$ be a neighborhood of $\nu$ in $P\left(X_{T}\right)$ and $\varepsilon>0$. Replacing $T$ by a higher order block presentation if necessary, there is a $\delta$ with $0<\delta<\varepsilon$ so that if $\left|\nu^{\prime}\left(a^{*}\right)-\nu\left(a^{*}\right)\right|<\delta$ for all $a \in \mathcal{L}_{T}$, then $\nu^{\prime} \in V$. Since $Z$ is proper, there is a $D \in B_{d}\left(X_{T}\right)$ so that $D^{*} \cap Z=\varnothing$, where we continue to use the notation $D^{*}=\left\{x \in X_{T}: x[0, d-1]=D\right\}$. Let $l$ be a transition length for $T$, and let $\gamma>0$ whose value will be determined later. By the ergodic and Shannon-McMillanBreiman theorems, there is an $m$ large enough so $m /(2 l+d+m+1)>1-\delta / 3$ and collections $C_{j} \subset B_{j}\left(X_{T}\right) \quad(j=m, m+1)$ so that

$$
\exp [m(h(\nu)-\gamma)]<\left|C_{j}\right|<\exp [m h(\nu)]
$$

and

$$
\left|f_{a}(C)-\nu\left(a^{*}\right)\right|<\frac{\delta}{3} \quad \text { for all } C \in C_{m} \cup C_{m+1},
$$

where $f_{a}(C)$ denote the frequency of the symbol $a$ in $C$. For each $C \in C=$ $C_{m} \cup C_{m+1}$ choose $A_{0}, A_{1} \in B_{l}\left(X_{T}\right)$ with $C^{\prime}=A_{0} C A_{1}$ so that $D C^{\prime} D \in B\left(X_{T}\right)$. Form the collection $C^{\prime}$ of such blocks. Let $Y$ be the shift of finite type with the subblocks of concatenations of elements of $D C^{\prime}$ as allowed blocks. Clearly $Y$ is topologically mixing since $m$ and $m+1$ are relatively prime. Let $\bar{\nu}$ be the measure of maximal entropy on $Y$. We show first that $|h(\bar{\nu})-h(\nu)|<\varepsilon$ and $\operatorname{supp}(\bar{\nu}) \cap Z=\varnothing$, then perturb $\bar{\nu}$ to get singularity with respect to $\mu$.

First note that $Y \subset \bigcup_{j=0}^{2 l+d+m} \sigma_{T}^{j}\left(D^{*}\right)$ and $Z \cap \sigma_{T}^{j}\left(D^{*}\right)=\varnothing$ for all $j$, so $Y \cap Z=\varnothing$.

Let $\nu^{\prime}$ be any $\sigma_{T}$-invariant measure with $\nu^{\prime}(Y)=1$. By the ergodic theorem, there is a $y \in Y$ so that $y[0, d-1]=D$ and $\left|f_{a}(y[0, n-1])-\nu^{\prime}\left(a^{*}\right)\right|<\delta / 3$ for all $a \in \mathcal{L}_{T}$ and sufficiently large $n$. Pick $n$ so that $y[0, n-1]$ has the form $D C_{1}^{\prime} D C_{2}^{\prime} \cdots D C_{k}^{\prime}, C_{j}^{\prime} \in C^{\prime}$. Then $k>n /(2 l+d+m)$, and if $C_{j}^{\prime}$ has central block 
$C_{j}$ then

$$
\begin{aligned}
\left|n f_{a}(y[0, n-1])-n \nu\left(a^{*}\right)\right| & \leq(2 d+l) k+\sum_{j=1}^{k}\left|C_{j}\right|\left(f_{a}\left(C_{j}\right)-\nu\left(a^{*}\right)\right) \\
& \leq\left(\frac{2 l+d}{2 l+d+m}\right) n+\frac{\delta}{3} n<\frac{2}{3} \delta n .
\end{aligned}
$$

In particular, $\left|\bar{\nu}\left(a^{*}\right)-\nu\left(a^{*}\right)\right|<\delta$ for $a \in \mathcal{L}_{T}$, proving $\bar{\nu} \in V$.

Next we estimate $h(\bar{\nu})=h\left(\left.\sigma_{T}\right|_{Y}\right)$ by estimating $\left|B_{n}(Y)\right|$. Every $E \in B_{n}(Y)$ has the form $E=A D C_{1}^{\prime} D C_{2}^{\prime} \cdots D C_{k}^{\prime} B$, where $|A|,|B| \leq 2 l+d+m, C_{j}^{\prime} \in C^{\prime}$, and

$$
\frac{n}{2 l+d+m+1}-2 \leq k \leq \frac{n}{2 l+d+m} .
$$

Making independent choices of the $C_{j}^{\prime}$, it follows from (10-1) that

$$
\begin{aligned}
\left|B_{n}(Y)\right| & \geq(\exp [m(h(\nu)-\gamma)])^{k} \\
& \geq \kappa_{1} \exp \left[\left(\frac{m}{2 l+d+m+1}\right) n(h(\nu)-\gamma)\right] \\
& \geq \kappa_{1} \exp \left[\left(1-\frac{\delta}{3}\right) n(h(\nu)-\gamma)\right],
\end{aligned}
$$

where $\kappa_{1}>0$. Hence

$$
h\left(\left.\sigma_{T}\right|_{Y}\right)=\lim _{n \rightarrow \infty} \frac{1}{n} \log \left|B_{n}(Y)\right| \geq\left(1-\frac{\delta}{3}\right)(h(\nu)-\gamma)>h(\nu)-\varepsilon
$$

for $\gamma$ small enough. Similar estimates show

$$
\begin{aligned}
\left|B_{n}(Y)\right| & \leq\left|B_{2 l+d+m}\left(X_{T}\right)\right|^{2}(\exp [m h(\nu)])^{k} \\
& \leq \kappa_{2} \exp \left[\left(\frac{m}{2 l+d+m}\right) n h(\nu)\right]
\end{aligned}
$$

for suitable $\kappa_{2}>0$, showing $h\left(\left.\sigma_{T}\right|_{Y}\right) \leq h(\nu)$. Thus $h(\nu)-\varepsilon<h(\bar{\nu}) \leq h(\nu)$.

We have hence found a mixing Markov measure $\bar{\nu} \in V$ so that $|h(\bar{\nu})-h(\nu)|<\varepsilon$ and $\operatorname{supp}(\bar{\nu}) \cap Z=\varnothing$. By perturbing the transition probabilities of $\bar{\nu}$, we can obtain a continuous family $\left\{\bar{\nu}_{\alpha}\right\}$ of mixing Markov measures each supported on $Y$ and with $\bar{\nu}_{0}=\bar{\nu}$. For small enough $\alpha$ the $\bar{\nu}_{\alpha}$ remain in $V$ and have $\left|h\left(\bar{\nu}_{\alpha}\right)-h(\nu)\right|<\varepsilon$. Now the $\bar{\nu}_{\alpha}$ are ergodic and distinct, so they are mutually singular. It follows that all but countably many of the $\bar{\nu}_{\alpha}$ are singular with respect to $\mu$. Set $\tilde{\nu}$ to be any of these.

If $\mu$ has ergodic decomposition $\mu=\int_{0}^{1} \mu^{t} d t$, define $h_{\infty}(\mu)=\operatorname{ess}_{\sup _{0 \leq t \leq 1}} h\left(\mu^{t}\right)$.

Convex APproximation Lemma 10.4. Let $\mu, \nu \in P\left(X_{T}\right)$ with $H_{\mu} \leq H_{\nu}$ and $h_{\infty}(\mu)<h\left(\sigma_{T}\right)$, and let $Z$ be a proper subshift. For every neighborhood $V$ of $\nu$ in $P\left(X_{T}\right)$ there is a $\tilde{\nu}=\sum_{k=1}^{K} \alpha_{k} \tilde{\nu}_{k} \in V$ and a corresponding convex decomposition $\mu=\sum_{k=1}^{K} \alpha_{k} \mu_{k}$, where $\alpha_{k}>0$ with $\sum_{k=1}^{K} \alpha_{k}=1$, such that the $\mu_{k}$ and $\tilde{\nu}_{k}$ are all mutually disjoint probability measures, the $\tilde{\nu}_{k}$ are mixing Markov measures with disjoint supports that are also disjoint from $Z$, and $h_{\infty}\left(\mu_{k}\right)<h\left(\tilde{\nu}_{k}\right)$.

PROOF. Let $\mu=\int_{0}^{1} \mu^{t} d t$ and $\nu=\int_{0}^{1} \nu^{t} d t$ be the monotone ergodic decompositions as described above. Put $\beta=h\left(\sigma_{T}\right)-h_{\infty}(\mu)>0$. Choose $\varepsilon>0$ so that 
if $\nu^{\prime} \in P\left(X_{T}\right)$ and $\left\|\nu-\nu^{\prime}\right\|<2 \varepsilon$, then $\nu^{\prime} \in V$. By partitioning [0,h( $\left.\left.\sigma_{T}\right)\right]$ into intervals of length $<\varepsilon \beta / 2$, we can find $0=t_{0}<t_{1}<\cdots<t_{K-1}<t_{K}=1$ so that if $J_{k}=\left[t_{k-1}, t_{k}\right)$ and $\mu_{k}=\left|J_{k}\right|^{-1} \int_{J_{k}} \mu^{t} d t$, then the $\mu_{k}$ are mutually singular and

$$
\sup _{t \in J_{k}}\left\{h\left(\mu^{t}\right)\right\}-\inf _{t \in J_{k}}\left\{h\left(\mu^{t}\right)\right\}<\varepsilon \beta / 2 \text {. }
$$

Put $\alpha_{k}=\left|J_{k}\right|$. We now inductively define $\nu_{k} \in P\left(X_{T}\right)$ so that $\nu=\sum_{k=1}^{K} \alpha_{k} \nu_{k}$ and $h\left(\nu_{k}\right) \geq h\left(\mu_{k}\right)$. Let

$$
s_{1}=\sup \left\{s \leq 1-t_{1}: \int_{s}^{s+t_{1}} h\left(\nu^{t}\right) d t \geq \int_{0}^{t_{1}} h\left(\mu^{t}\right) d t\right\} .
$$

Since $H_{\nu}\left(t_{1}\right) \geq H_{\mu}\left(t_{1}\right)$, the set defining $s_{1}$ is nonvoid. Put $\nu_{1}=t_{1}^{-1} \int_{s_{1}}^{s_{1}+t_{1}} \nu^{t} d t$. By definition $h\left(\nu_{1}\right) \geq h\left(\mu_{1}\right)$, and we claim $H_{\nu-\nu_{1}} \geq H_{\mu-\mu_{1}}$ on $\left[0,1-t_{1}\right]$, which allows inductive construction of all the $\nu_{k}$. To prove the claim, first suppose $s_{1}=1-t_{1}$. Then using monotonicity

$$
\begin{aligned}
\inf _{0 \leq t \leq 1-t_{1}}\left\{h\left(\nu^{t}\right)\right\} & \geq \frac{1}{t_{1}} \int_{1-t_{1}}^{1} h\left(\nu^{t}\right) d t \\
& \geq \frac{1}{t_{1}} \int_{0}^{t_{1}} h\left(\mu^{t}\right) d t \geq \sup _{t_{1} \leq t \leq 1}\left\{h\left(\mu^{t}\right)\right\},
\end{aligned}
$$

so $H_{\nu-\nu_{1}} \geq H_{\mu-\mu_{1}}$ follows trivially. Now assume $s_{1}<1-t_{1}$, so by continuity $\int_{s_{1}}^{s_{1}+t_{1}} h\left(\nu^{t}\right) d t=\int_{0}^{t_{1}} h\left(\mu^{t}\right) d t$. If $u<s_{1}$, then

$$
\begin{aligned}
H_{\nu-\nu_{1}}(u) & =\int_{0}^{u} h\left(\nu^{t}\right) d t \geq \int_{0}^{u} h\left(\mu^{t}\right) d t \\
& \geq \int_{t_{1}}^{t_{1}+u} h\left(\mu^{t}\right) d t=H_{\mu-\mu_{1}}(u),
\end{aligned}
$$

while if $s_{1}<u<1-t$, then

$$
\begin{aligned}
H_{\nu-\nu_{1}}(u) & =\int_{0}^{t_{1}+u} h\left(\nu^{t}\right) d t-\int_{s_{1}}^{s_{1}+t_{1}} h\left(\nu^{t}\right) d t \\
& \geq \int_{0}^{t_{1}+u} h\left(\mu^{t}\right) d t-\int_{0}^{t_{1}} h\left(\mu^{t}\right) d t=H_{\mu-\mu_{1}}(u),
\end{aligned}
$$

verifying our claim.

For each $k$ let $\nu_{k}=\int_{0}^{1} \nu_{k}^{t} d t$ be the ergodic decomposition, put $\nu_{k}^{\prime}=\int_{0}^{1-\varepsilon} \nu_{k}^{t} d t+$ $\varepsilon \mu_{T}$, and sum $\nu^{\prime}=\sum_{k=1}^{K} \nu_{k}^{\prime}$. We show $h\left(\nu_{k}^{\prime}\right)>h_{\infty}\left(\mu_{k}\right)$. For by $(10-3)$

$$
\begin{aligned}
h\left(\nu_{k}^{\prime}\right) & =h\left(\nu_{k}\right)-\int_{1-\varepsilon}^{1} h\left(\nu_{k}^{t}\right) d t+\varepsilon h\left(\sigma_{T}\right) \\
& \geq h\left(\mu_{k}\right)-\varepsilon h_{\infty}\left(\mu_{k}\right)+\varepsilon h\left(\sigma_{T}\right) \\
& \geq h_{\infty}\left(\mu_{k}\right)+\varepsilon \beta / 2>h_{\infty}\left(\mu_{k}\right) .
\end{aligned}
$$

Since $\left\|\nu-\nu^{\prime}\right\| \leq 2 \varepsilon$, it follows by our choice of $\varepsilon$ that $\nu^{\prime} \in V$. Finally, by Lemma 10.3 we can inductively modify each $\nu_{k}^{\prime}$ to $\tilde{\nu}_{k}$ so that $\tilde{\nu}=\sum_{k=1}^{K} \alpha_{k} \tilde{\nu}_{k}$ satisfies the conclusions.

If $F \subset X_{T}$ and $x \in F$, define $r_{F}(x)=\min \left\{n>0: \sigma_{T}^{n} x \in F\right\}$, and put $F_{n}=\{x \in$ $\left.F: r_{F}(x)=n\right\}$. For a collection $C \subset B\left(X_{T}\right)$ let $C^{*}=\bigcup_{C \in C} C^{*}$. 
LEMMA 10.5. Suppose $\mu_{k} \in P\left(X_{T}\right)$ for $1 \leq k \leq K$ are mutually singular, and $\varepsilon>0$. There is an $M=M\left(\varepsilon, \mu_{1}, \ldots, \mu_{K}\right)$ so that if $F$ is compact open and $N \geq n \geq M$, then there are collections $C_{n}\left(\mu_{k}\right) \subset B_{n}\left(X_{T}\right)$, disjoint in $n$ and $k$, such that $\left|C_{n}\left(\mu_{k}\right)\right|<\exp \left[n\left(h_{\infty}\left(\mu_{k}\right)+\varepsilon\right)\right]$ for $1 \leq k \leq K$ and

$$
\sum_{n=M}^{N} n \mu_{k}\left(F_{n} \cap \mathcal{C}_{n}\left(\mu_{k}\right)^{*}\right) \geq \sum_{n=M}^{N} n \mu_{k}\left(F_{n}\right)-\varepsilon .
$$

ProOF. The rough idea is to use singularity of the $\mu_{k}$ to first find disjoint collections $D_{k}$ of atoms having the right exponential size and containing most of the $\mu_{k}$-mass. Using the idea that a partial orbit can intersect at most one of these atoms with frequency $>\frac{1}{2}$, we obtain from the $D_{k}$ the required disjoint collections $C_{n}\left(\mu_{k}\right)$.

Since the $\mu_{k}$ are mutually singular, there is an $s$ and disjoint collections $D_{k} \subset$ $B_{2 s+1}\left(X_{T}\right)$ such that $\mu_{k}\left(D_{k}^{*}\right)>1-\varepsilon / 12$ for $1 \leq k \leq K$. Applying the version of the Shannon-McMillan-Breiman theorem from [DGS, Theorem 13.4] to $\sigma_{T}$ and to $\sigma_{T}^{-1}$, for all large enough $M$ and $1 \leq k \leq K$ we have

$$
\begin{aligned}
& \mu_{k}\left(E_{k}^{+}\right)=\mu_{k}\left\{x: \sup _{m \geq M / 6}-\frac{1}{m} \log \mu_{k}\left(x[0, m-1]^{*}\right)<h_{\infty}\left(\mu_{k}\right)+\frac{\varepsilon}{2}\right\}>1-\frac{\varepsilon}{12}, \\
& \mu_{k}\left(E_{k}^{-}\right)=\mu_{k}\left\{x: \sup _{m \geq M / 6}-\frac{1}{m} \log \mu_{k}\left(x[-m+1,0]^{*}\right)<h_{\infty}\left(\mu_{k}\right)+\frac{\varepsilon}{2}\right\}>1-\frac{\varepsilon}{12} .
\end{aligned}
$$

Let $E_{k}=E_{k}^{+} \cap E_{k}^{-}$, and fix $M$ so that $\mu_{k}\left(E_{k}\right)>1-\varepsilon / 6$, and also satisfying $M>6 s$ and

$$
\frac{n}{3} \exp \left[h_{\infty}\left(\mu_{k}\right)+(n+1) \frac{\varepsilon}{2}\right]<\exp [n \varepsilon]
$$

for $n \geq M / 6$ and $1 \leq k \leq K$.

Suppose now $F$ is compact open, and $M<n<N$. If $x \in F_{n}$ with $\sigma_{T}^{j} x \in E_{k}$ for some $n / 3 \leq j \leq 2 n / 3$, then

$$
\mu_{k}\left(x[j, n-1]^{*}\right)>\exp \left[-(n-j)\left(h_{\infty}\left(\mu_{k}\right)+\frac{\varepsilon}{2}\right)\right] .
$$

The number of blocks $x[j, n-1]$ arising from such an $x$ is thus bounded above by $\exp \left[(n-j)\left(h_{\infty}\left(\mu_{k}\right)+\varepsilon / 2\right)\right]$. Similarly the number of blocks $x[0, j]$ from such an $x$ is $\leq \exp \left[(j+1)\left(h_{\infty}\left(\mu_{k}\right)+\varepsilon / 2\right)\right]$. It follows using (10-4) that the number of blocks $x[0, n-1]$, where $x \in F_{n}$ and $\sigma_{T}^{j} x \in E_{k}$ for some $n / 2 \leq j \leq 2 n / 3$ is bounded above by

$$
\frac{n}{3} \exp \left[(n+1)\left(h_{\infty}\left(\mu_{k}\right)+\frac{\varepsilon}{2}\right)\right] \leq \exp \left[n\left(h_{\infty}\left(\mu_{k}\right)+\varepsilon\right)\right] .
$$

Now let $C_{n}\left(\mu_{k}\right)$ denote the collection of blocks $x[0, n-1]$, where $x \in F_{n}, \sigma_{T}^{j} x \in E_{k}$ for some $n / 3 \leq j \leq 2 n / 3$, and

$$
\left|\left\{j: \frac{n}{3} \leq j \leq \frac{2 n}{3}, x[j-s, j+s] \in D_{k}\right\}\right|>\frac{n}{6} .
$$

Since the $D_{k}$ are disjoint, clearly the $C_{n}\left(\mu_{k}\right)$ are disjoint in $k$ and trivially in $n$ since the lengths differ. The discussion above proves $\left|C_{n}\left(\mu_{k}\right)\right|<\exp \left[n\left(h_{\infty}\left(\mu_{k}\right)+\varepsilon\right)\right]$. 
Finally, since

$$
\begin{aligned}
& n \mu_{k}\left\{x \in F_{n}: x[0, n-1] \notin C_{n}\left(\mu_{k}\right)\right\} \\
& \leq 3 \mu_{k}\left(E_{k}^{c} \cap \bigcup_{j=n / 3}^{2 n / 3} \sigma_{T}^{j} F_{n}\right)+6 \mu_{k}\left(D_{k}^{* c} \cap \bigcup_{j=n / 3}^{2 n / 3} \sigma_{T}^{j} F_{n}\right),
\end{aligned}
$$

we have

$$
\begin{aligned}
\sum_{n=M}^{N} n \mu_{k}\left(F_{n} \cap C_{n}\left(\mu_{k}\right)^{*}\right) & \geq \sum_{n=M}^{N} n \mu_{k}\left(F_{n}\right)-3 \mu_{k}\left(E_{k}^{c}\right)-6 \mu_{k}\left(D_{k}^{* c}\right) \\
& \geq \sum_{n=M}^{N} n \mu_{k}\left(F_{n}\right)-\varepsilon .
\end{aligned}
$$

LEMMA 10.6. Suppose $\mu, \nu \in P\left(X_{T}\right)$ with $H_{\mu} \leq H_{\nu}$, that $\mu$ is continuous, and that $\operatorname{supp}\left(\mu^{t}\right)=X_{T}$ for a.e. $t$. Let $Z$ be a proper subshift of $X_{T}$ and $V$ be a neighborhood of $\nu$ in $P\left(X_{T}\right)$. Then there is an involution $\varphi \in \operatorname{aut}\left(\sigma_{T}\right)$ such that $\varphi \mu \in V$ and $\varphi$ is the identity on $Z$.

ProOF. We first reduce to the case $h_{\infty}(\mu)<h\left(\sigma_{T}\right)$. Let $t_{0}=\sup \left\{t: h\left(\mu^{t}\right)=\right.$ $\left.h\left(\sigma_{T}\right)\right\}$. Since $\sigma_{T}$ is intrinsically ergodic, for $0 \leq t \leq t_{0}$ we have $\mu^{t}=\nu^{t}=\mu_{T}$. Since $\mu_{T}$ is $G$-invariant, any $\varphi \in G$, in particular the $\varphi$ we construct below, maps $\int_{0}^{t_{0}} \mu^{t} d t=t_{0} \mu_{T}$ to $\int_{0}^{t_{0}} \nu^{t} d t$. Removing this part from each, we are reduced to the case $h\left(\mu^{t}\right)<h\left(\sigma_{T}\right)$ for all $t$. Since it is enough to map $(1-\varepsilon)^{-1} \int_{\varepsilon}^{1} \mu^{t} d t$ to $V$ for a sufficiently small $\varepsilon>0$, we can and do assume $h_{\infty}(\mu)<h\left(\sigma_{T}\right)$.

Using the Convex Approximation Lemma, there is $\tilde{\nu}=\sum_{k=1}^{K} \alpha_{k} \tilde{\nu}_{k} \in V$ and $\mu=\sum_{k=1}^{K} \alpha_{k} \mu_{k}$ with $\alpha_{k}>0, \sum_{k=1}^{K} \alpha_{k}=1$, so that the $\mu_{k}$ and $\tilde{\nu}_{k}$ are all mutually disjoint, the $\tilde{\nu}_{k}$ are mixing Markov measures with supports $Y_{k}$ that are mutually disjoint and disjoint from $Z$, and $h_{\infty}\left(\mu_{k}\right)<h\left(\tilde{\nu}_{k}\right)$.

By passing to a higher block presentation, we can assume that the $\left.\sigma_{T}\right|_{Y_{k}}$ and $\sigma_{T}$ are 1-step, $\mathcal{L}_{Y_{k}}$ and $\mathcal{L}_{Z}$ are all disjoint, and that there is a $\delta_{0}>0$ so that if $\left|\nu^{\prime}\left(a^{*}\right)-\tilde{\nu}\left(a^{*}\right)\right|<\delta_{0}$, then $\nu^{\prime} \in V$. Let $\delta>0$, which will eventually be made small enough for everything which follows to work. The first requirement on $\delta$ is that

$$
(1-\delta)\left[h\left(\tilde{\nu}_{k}\right)-\delta\right]>h\left(\mu_{k}\right)+\delta
$$

for all $k$.

By the ergodic and Shannon-McMillan-Breiman theorems, there exists an $M_{0}$ so that for $n>M_{0}$ there is a collection $C_{n}\left(\tilde{\nu}_{k}\right) \subset B_{n}\left(Y_{k}\right)$ so that

$$
\begin{gathered}
\left|C_{n}\left(\tilde{\nu}_{k}\right)\right|>\exp \left[n\left(h\left(\tilde{\nu}_{k}\right)-\delta\right)\right], \\
\tilde{\nu}_{k}\left(C_{n}\left(\tilde{\nu}_{k}\right)^{*}\right)>1-\delta
\end{gathered}
$$

and

$$
\left|f_{a}(C)-\tilde{\nu}_{k}\left(a^{*}\right)\right|<\delta \quad \text { for all } a \in \mathcal{L}_{T} \text {. }
$$

There is a block $A_{0} \in B\left(X_{T}\right)$ so that $A_{0}^{*} \cap\left(Z \cup \cup_{k=1}^{K} Y_{k}\right)=\varnothing$. Since the number of blocks in $B_{n}\left(X_{T}\right)$ beginning with $A_{0}$ grows exponentially in $n$, and each $\mu_{k}$ has full support, there is a block $A$ beginning with $A_{0}$ so that $0<\mu_{k}\left(A^{*}\right)<\delta^{2} / 4|A|$ 
for $1 \leq k \leq K$. Let $|A|=d$ and put $M=[d / 4 \delta]$. Let $l$ be a transition length for each $\left.\sigma_{T}\right|_{Y_{k}}$ and for $\sigma_{T}$. We may assume $d$ is large enough so that $M \geq M_{0}$, that $M \geq M\left(\delta, \mu_{1}, \ldots, \mu_{K}\right)$ from Lemma 10.5 , and that $n-2 d-4 l>(1-\delta) n$ for $n \geq M$. Since $\operatorname{supp}\left(\mu_{k}\right)=X_{T}$, there is an $N>M$ so that $\mu_{k}\left(\bigcup_{j=0}^{N-1} \sigma_{T}^{j} A^{*}\right)>1-\delta$ for $1 \leq k \leq K$. Let $F=A^{*}$. If $F_{n}=\left\{x \in F: r_{F}(x)=n\right\}$, then

$$
\sum_{n=M}^{N} n \mu_{k}\left(F_{n}\right)>1-M \mu_{k}\left(A^{*}\right)-\delta>1-2 \delta .
$$

By Lemma 10.5, there are mutually disjoint collections $C_{n}\left(\mu_{k}\right) \subset B_{n}\left(X_{T}\right)$ so that $\left|C_{n}\left(\mu_{k}\right)\right| \leq \exp \left[n\left(h_{\infty}\left(\mu_{k}\right)+\delta\right)\right]$ and

$$
\sum_{n=M}^{N} n \mu_{k}\left(F_{n} \cap C_{n}\left(\mu_{k}\right)^{*}\right)>1-3 \delta
$$

We now construct $\varphi$. For each $k$ choose a block $A_{k}$ so $A A_{k} \in B\left(X_{T}\right)$ has minimal length such that it ends with a block from $B_{d}\left(Y_{k}\right)$. Note that $\left|A_{k}\right|<d$ is possible. Clearly $\left|A_{k}\right|<2 d+l$ since $l$ is a transition length for $\sigma_{T}$. Similarly choose $B_{k}$ so $B_{k} A \in B\left(X_{T}\right)$ and has minimal length for such a block starting with a block from $B_{d}\left(Y_{k}\right)$. For each $C \in C_{n-3 d-4 l}\left(\tilde{\nu}_{k}\right)$ there is a $C^{\prime}$ containing $C$ as a subblock so that $A A_{k} C^{\prime} B_{k} A \in B_{n+d}\left(X_{T}\right)$, and so that $A$ occurs only as the initial and terminal $d$-block. Form $D_{n k}=\left\{A A_{k} C^{\prime} B_{k} A: C \in C_{n-3 d-4 l}\left(\tilde{\nu}_{k}\right)\right\}$, so

$$
\begin{aligned}
\left|D_{n k}\right| & =\left|C_{n-3 d-4 l}\left(\tilde{\nu}_{k}\right)\right|>\exp \left[(n-3 d-4 l)\left(h\left(\tilde{\nu}_{k}\right)-\delta\right)\right] \\
& >\exp \left[n(1-\delta)\left(h\left(\tilde{\nu}_{k}\right)-\delta\right)\right] \\
& >\exp \left[n\left(h_{\infty}\left(\mu_{k}\right)+\delta\right)\right]>\left|C_{n}\left(\mu_{k}\right)\right| .
\end{aligned}
$$

Thus it is possible to define a permutation $\theta_{n k}$ of $A B_{n-d}\left(X_{T}\right) A$ so that $\theta_{n k}^{2}=I$, $\theta_{n k}\left(C_{n}\left(\mu_{k}\right) A\right) \subset D_{n k}$, and $\theta_{n k}$ is the identity off $C_{n}\left(\mu_{k}\right) A \cup \theta_{n k}\left(C_{n}\left(\mu_{k}\right) A\right)$ Note that each block in $C_{n}\left(\mu_{k}\right)$ begins with $A$, so these $\theta_{n k}$ are exactly the type introduced in $\S 2$, where here $A$ is the marker block.

If $x \in X_{T}$ with $x[i, i+n+d] \in C_{n}\left(\mu_{k}\right) A \cup \theta_{n k}\left(C_{n}\left(\mu_{k}\right) A\right)$, where $M \leq n \leq N$ and $1 \leq k \leq K$, define $(\varphi x)([i, i+n+d])=\theta_{n k}(x[i, i+n+d])$. Since the $C_{n}\left(\mu_{k}\right)$ are disjoint in $n$ and $k$, and $\theta_{n k}^{2}=I$, it follows that $\varphi$ is a well-defined involution.

We complete this proof by showing that $\left|\varphi \mu_{k}\left(a^{*}\right)-\tilde{\nu}_{k}\left(a^{*}\right)\right|<\delta_{0}$ for each $k$ and $a \in \mathcal{L}_{T}$. This will imply that $\left|\varphi \mu\left(a^{*}\right)-\tilde{\nu}\left(a^{*}\right)\right|<\delta_{0}$ for $a \in \mathcal{L}_{T}$, so $\varphi \mu \in V$, the required conclusion. If $C \in C_{n}\left(\mu_{k}\right)$ then

$$
\begin{aligned}
\varphi \mu_{k}\left(a^{*} \cap \bigcup_{j=0}^{n-1} \sigma_{T}^{i}\left(F_{n} \cap \theta_{n k}\left(C^{*}\right)\right)\right) & =n f_{a}\left(\theta_{n k}(C)\right) \varphi \mu_{k}\left(F_{n} \cap \theta_{n k}(C)^{*}\right) \\
& =n f_{a}\left(\theta_{n k}(C)\right) \mu_{k}\left(F_{n} \cap C^{*}\right) .
\end{aligned}
$$

Letting

$$
E=\bigcup_{n=M}^{N} \bigcup_{j=0}^{n-1} \bigcup_{C \in C_{n}\left(\mu_{k}\right)} \sigma_{T}^{j}\left(F_{n} \cap \theta_{n k}(C)^{*}\right),
$$

then (10-7) above shows that $\varphi \mu_{k}(E)>1-3 \delta$ while by (10-6) $\mid \varphi \mu_{k}\left(a^{*} \cap E\right)-$ $\tilde{\nu}_{k}\left(a^{*}\right) \mid<2 \delta$. For $\delta$ sufficiently small this forces $\left|\varphi \mu_{k}\left(a^{*}\right)-\tilde{\nu}_{k}\left(a^{*}\right)\right|<\delta_{0}$, concluding the proof. 
The following result deals with the case that $\mu$ does not have full support. Complications arise because $\mu$ may be highly recurrent, so that constructing markers with the necessary disjointness is more difficult. To appreciate the problems, an excellent example to keep in mind while reading the proof is for $\mu$ to be the unique invariant measure supported on the Morse minimal set and $\nu$ to be the measure of maximal entropy on $X_{[2]}$. In this sense, the difficulties parallel those in the second half of the proof of Theorem 9.2.

LEMMA 10.7. Suppose $\mu, \nu \in P\left(X_{T}\right)$ with $H_{\mu} \leq H_{\nu}$, that $\mu$ is continuous, and $\operatorname{supp}(\mu) \neq X_{T}$. For every neighborhood $V$ of $\nu$ in $P\left(X_{T}\right)$ there is an involution $\varphi \in \operatorname{aut}\left(\sigma_{T}\right)$ with $\varphi \mu \in V$.

ProOF. Let $Z=\operatorname{supp}(\mu) \neq X_{T}$. Since $\operatorname{supp}\left(\mu^{t}\right) \subset Z$ for a.e. $t$, it follows that $h_{\infty}(\mu) \leq h\left(\left.\sigma_{T}\right|_{Z}\right)<h\left(\sigma_{T}\right)$. By the Convex Approximation Lemma, there is a $\tilde{\nu}=\sum_{k=1}^{K} \alpha_{k} \tilde{\nu}_{k} \in V$ and $\mu=\sum_{k=1}^{K} \alpha_{k} \mu_{k}$, where $\alpha_{k}>0$ with $\sum_{k=1}^{K} \alpha_{k}=1$, the $\mu_{k}$ and $\tilde{\nu}_{k}$ are all mutually singular, each $\tilde{\nu}_{k}$ is mixing Markov supported on a mixing shift of finite type $Y_{k}$ with the $Y_{k}$ and $Z$ mutually disjoint, and $h_{\infty}\left(\mu_{k}\right)<h\left(\tilde{\nu}_{k}\right)$ for all $k$.

By passing to a higher order block presentation, we may arrange $\mathcal{L}_{Y_{k}}$ and $\mathcal{L}_{Z}$ to be disjoint, $\sigma_{T} \mid Y_{k}$ and $\sigma_{T}$ to be 1-step, and for there to exist a $\delta_{0}>0$ so that if $\left|\nu^{\prime}\left(a^{*}\right)-\tilde{\nu}\left(a^{*}\right)\right|<\delta_{0}$, then $\nu^{\prime} \in V$. Introduce $\delta>0$, which will eventually be made small enough for the following to work.

Before starting the main argument, we first recode $\sigma_{T}$ so that for each $a \in \mathcal{L}_{Z}$ and all $k$ there are "escape" blocks $A_{a k}$ and $A_{k a}$ so that $A_{a k}$ starts with $a$, has no other symbol in $\mathcal{L}_{Z}$, and ends with a symbol from $\mathcal{L}_{Y_{k}}$, while $A_{k a}$ has these properties in reverse order. To arrange this recoding, first choose $A_{a k}^{\prime}$ starting with $a$, no other symbol is $a$, and ending with a symbol from $\mathcal{L}_{Y_{k}}$. Choose $A_{k a}^{\prime}$ to have these properties in reverse order. Introduce entirely new distinct symbols $b_{a k}, b_{k a} \notin \mathcal{L}_{T}$ for all $a$ and $k$. Form a new shift of finite type from $X_{T}$ by replacing all symbols in $A_{a k}^{\prime}$ except the first and last by the same number of $b_{a k}$ 's, and similarly with the $A_{k a}^{\prime}$ and $b_{k a}$ 's. This recoding of $X_{T}$ has the property sought, but is not 1-step. Pass to a higher order block presentation so the resulting shift is 1-step, and the existence of the escape blocks as above is preserved.

By the ergodic and Shannon-McMillan-Breiman theorems, there is an $M_{0}$ so that for $n \geq M_{0}$ and all $k$ there is a collection $C_{n}\left(\tilde{\nu}_{k}\right) \subset B_{n}\left(Y_{k}\right)$ so that

$$
\tilde{\nu}_{k}\left(C_{n}\left(\tilde{\nu}_{k}\right)^{*}\right)>1-\delta, \quad\left|C_{n}\left(\tilde{\nu}_{k}\right)\right|>\exp \left[n\left(h\left(\tilde{\nu}_{k}\right)-\delta\right)\right], \quad \text { and }
$$

$$
\left|f_{a}(C)-\tilde{\nu}_{k}\left(a^{*}\right)\right|<\delta \quad \text { for all } C \in \mathcal{C}_{n}\left(\tilde{\nu}_{k}\right) \text { and } a \in \mathcal{L}_{T}
$$

Let $M_{1}=M\left(\delta, \mu_{1}, \ldots, \mu_{K}\right)$ from Lemma 10.5 , and let $l$ be a transition length for $\sigma_{T}$ and each $\left.\sigma_{T}\right|_{Y_{k}}$. Now fix $M>\max \left\{4 l / \delta, M_{0}, M_{1}\right\}$. For $n \geq M$, each $c, d \in \mathcal{L}_{Z}$, and $C \in C_{n-4 l}\left(\tilde{\nu}_{K}\right)$ there is a block $A_{c k} C^{\prime} A_{k d} \in B_{n}\left(X_{T}\right)$ with $C$ a subblock of $C^{\prime}$. Form the collection $D_{n k}(c, d)$ of such blocks. Note that each $D \in D_{n k}(c, d)$ starts with $c$, ends with $d$, has no other symbols in $\mathcal{L}_{Z}$, and $\left|f_{a}(D)-\tilde{\nu}_{k}\left(a^{*}\right)\right|<2 \delta$ by (10-8). Also,

$$
\left|D_{n k}(c, d)\right|=\left|C_{n-4 l}\left(\tilde{\nu}_{k}\right)\right| \geq \exp \left[(n-4 l)\left(h\left(\tilde{\nu}_{k}\right)-\delta\right)\right]
$$


We now construct $\varphi$. Recall that $C \in B_{n}\left(X_{T}\right)$ is called $j$-periodic if $C[0, n-j]=$ $C[j, n]$. Since $\mu$ is continuous, there is a $p>M$ so that for all $k$,

$$
\mu_{k}\left(\bigcup\left\{C^{*}: C \in B_{2 p+1}\left(X_{T}\right) \text { is } j \text {-periodic for some } j<M\right\}\right)<\delta \text {. }
$$

By $[\mathbf{K r 1}]$ or [B1, Lemma 2.2], there is a compact open set $F \subset Z$ such that $\left\{\sigma_{T}^{j} F: 0 \leq j \leq M\right\}$ is disjoint, and

$$
\{x \in Z: x[-p, p] \text { is not } j \text {-periodic for all } j<M\} \subset \bigcup_{j=0}^{2 M-1} \sigma_{T}^{j} F \text {. }
$$

Recall that $r_{F}$ is the return time function for $F$, and put $F_{n}=\left\{x \in F: r_{F}(x)=n\right\}$. By the above we have

$$
\sum_{n=M}^{2 M-1} n \mu_{k}\left(F_{n}\right)>1-\delta
$$

Since $F$ is compact open, there is a $q>p$ so that $F$ is a union of sets $\sigma_{T}^{-q} B^{*}$ for $B \in B_{2 q+1}(Z)$. Let

$$
\mathcal{F}=\left\{A \in B_{2 q+4 M+1}(Z): \sigma_{T}^{-(q+2 M)} A^{*} \subset F\right\} .
$$

Since $M>M\left(\delta, \mu_{1}, \ldots, \mu_{k}\right)$ from Lemma 10.5, choose disjoint collections $C_{n}\left(\mu_{k}\right) \subset$ $B_{n}(Z)$ for $M \leq n<2 M$ and all $1 \leq k \leq K$ so that $\left|C_{n}\left(\mu_{k}\right)\right| \leq \exp \left[n\left(h_{\infty}\left(\mu_{k}\right)+\delta\right)\right]$ and

$$
\sum_{n=M}^{2 M-1} n \mu_{k}\left(F_{n} \cap C_{n}\left(\mu_{k}\right)^{*}\right)>1-2 \delta
$$

Thus for $\delta$ small enough $\left|\mathcal{C}_{n}\left(\mu_{k}\right)\right|<\left|D_{n k}(c, d)\right|$ for all $c, d \in \mathcal{L}_{Z}$. It follows there is a injection

$$
\theta_{n}: C_{n}\left(\mu_{k}\right) \rightarrow \bigcup_{c, d \in \mathcal{L}_{z}} D_{n k}(c, d)
$$

that fixes the first and last symbols.

Now define $\xi: B\left(X_{T}\right) \rightarrow B\left(X_{T}\right)$ to replace any occurrence of $\theta_{n}(C)$ by $C$, where $M \leq n \leq 2 M-1$ and $C \in C_{n}\left(\mu_{k}\right)$. This map is well-defined because blocks from $D_{n k}(c, d)$ can only overlap in the end symbols, and these symbols are fixed by $\theta_{n}$. Let

$$
\mathcal{E}=\left\{E[2 M, 2 q+6 M+1]: E \in \xi^{-1}\left(B_{2 M}(Z) \mathcal{F} B_{2 M}(Z)\right)\right\}
$$

and put $E=\sigma_{T}^{-(q+2 M)} \mathcal{E}^{*}$. We claim that $\left\{\sigma_{T}^{j} E: 0 \leq j<M\right\}$ is disjoint. For if $x=\sigma_{T}^{j} y$ for $x, y \in E$ and some $0 \leq j<M$, applying $\xi$ to $x[-q-2 M, q+2 M]$ and $y[-q-2 M-j, q+2 M-j]$ shows this would contradict disjointness of $\left\{\sigma_{T}^{j} F: 0 \leq\right.$ $j<M\}$. The mapping $\varphi$ is now defined as follows. If $\sigma_{T}^{i} x \in E$ and $\sigma_{T}^{i+n-1} \in E$ for $M \leq n<2 M$, then

$$
(\varphi x)[i, i+n-1]= \begin{cases}\theta_{n}(x[i, i+n-1]), & \text { if } x[i, i+n-1] \in C_{n}\left(\mu_{k}\right), \\ \theta_{n}^{-1}(x[i, i+n-1]), & \text { if } x[i, i+n-1] \in \theta_{n}\left(C_{n}\left(\mu_{k}\right)\right) .\end{cases}
$$

Because $\left\{\sigma_{T}^{j}: 0 \leq j<M\right\}$ is disjoint, $\varphi$ is well-defined. The definition of $\mathcal{E}$ is made so that $x[-q-2 M, q+2 M] \in \mathcal{E}$ iff $(\varphi x)[-q-2 M, q+2 M] \in \mathcal{E}$. Hence $\varphi^{2}=I$. Finally, the estimate that $\left|\varphi \mu\left(a^{*}\right)-\tilde{\nu}\left(a^{*}\right)\right|<\delta_{0}$ for all $a \in \mathcal{L}_{T}$ follows from (10-8) and (10-10) exactly as in the previous lemma. 
LEMMA 10.8. Let $\mu, \nu \in P\left(X_{T}\right)$ with $H_{\mu} \leq H_{\nu}$, let $E \subset[0,1]$ have positive measure, and set $\mu_{0}=\int_{E} \mu^{t} d t, \mu_{1}=\mu-\mu_{0}$. Then there is a decomposition $\nu=\nu_{0}+\nu_{1}$ with $H_{\nu_{j}} \geq H_{\mu_{j}}$ for $j=0,1$.

PROOF. Let $a$ be the measure of $E$, and put

$$
u_{0}=\sup \left\{u \leq 1-a: H_{\nu}(t+u)-H_{\nu}(u) \geq H_{\mu_{0}}(t) \text { for } 0 \leq t \leq a\right\} .
$$

The defining set clearly contains 0 , so is nonempty. Put $\nu_{0}=\int_{u_{0}}^{u_{0}+a} \nu^{t} d t$ and $\nu_{1}=\nu-\nu_{0}$. By definition, for $0 \leq t \leq a$,

$$
H_{\nu_{0}}(t)=H_{\nu}(t+u)-H_{\nu}(u) \geq H_{\mu_{0}}(t) .
$$

We now show $H_{\nu_{1}} \geq H_{\mu_{1}}$. If $u_{0}=1-a$, then since $h\left(\mu^{t}\right)$ is decreasing in $t$, for $0 \leq t \leq 1-a$ we have

$$
H_{\nu_{1}}(t)=H_{\nu}(t) \geq H_{\mu}(t) \geq H_{\mu_{1}}(t) .
$$

Now suppose $u_{0}<1-a$. Then by continuity

$$
\int_{u_{0}}^{u_{0}+a} h\left(\nu^{t}\right) d t=\int_{E} h\left(\mu^{t}\right) d t .
$$

If $t<u_{0}$, then (10-11) shows $H_{\nu_{1}}(t) \geq H_{\mu_{1}}(t)$. If $u_{0}<t \leq 1-a$, then using (10-12) we obtain

$$
\begin{aligned}
H_{\nu_{1}}(t) & =H_{\nu}(t+a)-\int_{u_{0}}^{u_{0}+a} h\left(\nu^{s}\right) d s \\
& \geq H_{\mu}(t+a)-\int_{E} h\left(\mu^{s}\right) d s \geq H_{\mu_{1}}(t) .
\end{aligned}
$$

PROOF OF THEOREM 10.1 (SUFficienCy). Suppose $\mu$ is continuous with $H_{\mu} \leq H_{\nu}$, and that $V$ is a neighborhood of $\nu$ in $P\left(X_{T}\right)$. There are $\varepsilon>0$ and a neighborhood $V_{0}$ of $\nu$ in $M\left(X_{T}\right)$ so that if $\nu^{\prime} \in V_{0}$ and $\nu^{\prime \prime} \in P\left(X_{T}\right)$, then $\left[(1-\varepsilon) \nu^{\prime}+\varepsilon \nu^{\prime \prime}\right] /\left[(1-\varepsilon) \nu^{\prime}\left(X_{T}\right)+\varepsilon\right] \in V$.

Since every proper subshift is contained in a proper shift of finite type, and there are only countably many of the latter, there is a proper subshift $Z \subset X_{T}$ such that

$$
m\left(E_{1}\right)=m\left(\left\{t \in[0,1]: \operatorname{supp}\left(\mu^{t}\right) \not \subset Z, \operatorname{supp}\left(\mu^{t}\right) \neq X_{T}\right\}\right)<\varepsilon,
$$

where $m$ is Lebesgue measure. Let $E_{0}=\left\{t \in[0,1]: \operatorname{supp}\left(\mu^{t}\right) \subset Z\right\}$ and $E_{2}=\{t \in$ $\left.[0,1]: \operatorname{supp}\left(\mu^{t}\right)=X_{T}\right\}$. These sets are clearly measurable. If $m\left(E_{0}\right)=0$, then by Lemma 10.6 all but $\varepsilon$ of $\mu$ can be mapped to a measure in $V_{0}$, and we are done.

Thus suppose $m\left(E_{0}\right)=a>0$. Let $\mu_{j}=\int_{E_{j}} \mu^{t} d t$. Two applications of Lemma 10.8 show that there is a decomposition $\nu=\nu_{0}+\nu_{1}+\nu_{2}$ with $H_{\nu_{j}} \geq H_{\mu_{j}}$ for $0 \leq j \leq 2$. By Lemma 10.7, there is a $\varphi_{1} \in G$ so that $\varphi_{1} \mu_{0}$ is close to $\nu_{0}$. By Lemma 10.6 , there is a $\varphi_{2} \in G$ that is the identity on $\varphi_{1}(Z)$ and that maps $\mu_{2}$ close to $\nu_{2}$. It follows that the $\varphi_{j}$ can be chosen so that $\varphi_{2} \varphi_{1}$ maps $\mu_{0}+\mu_{2}$ into $V_{0}$, and since $\mu_{2}\left(X_{T}\right)<\varepsilon$, we obtain finally $\left(\varphi_{2} \varphi_{1}\right) \mu \in V$.

11. Problems and questions. During the course of this paper we have indicated several open problems and questions. Two of these seem to us the most important. Recall that $F_{n}$ is the set of points in $X_{T}$ with period $\leq n$. 
QUESTION 6.10. Is the kernel of the dimension representation generated by elements of finite order?

QUESTION 7.1. When is an automorphism in $\operatorname{aut}\left(F_{n}, \sigma_{T}\right)$ the restriction of one in $\operatorname{aut}\left(X_{T}, \sigma_{T}\right)$ ?

Several others seem to be particularly interesting. Problem 3.3 asks whether there is an automorphism of infinite order with $n$th roots for infinitely many $n$. This is basic to understanding the kind of divisibility present in $G$. Our lack of knowledge about the algebraic structure of $G$ is pointed out in Question 4.1, which asks for a nontrivial case of two shifts of finite type having isomorphic automorphism groups. In particular, are the automorphism groups of the 2-shift and the 3-shift isomorphic? Problem 6.1 asks whether the dimension group representation $\delta$ of $G$ is always surjective. The $p$-adic behavior of the gyration function is quite interesting. Specifically (Question 8.5), does $g\left(\varphi, \sigma_{T}\right)\left(p^{n}\right)$ always converge $p$-adically for every $\varphi \in G$ ? Problem 9.6 generalizes Question 7.1 above to characterizing the orbits of subshifts of $X_{T}$.

\section{REFERENCES}

[ACH] R. Adler, D. Coppersmith, and M. Hassner, Algorithms for sliding block codes, IEEE Trans. on Information Theory 29 (1983), 5-22.

[Bo] R. Bowen, Topological entropy and Axiom A, Proc. Sympos. Pure Math., vol. 14, Amer. Math. Soc., Providence, R.I., 1970, pp. 23-41.

[B1] M. Boyle, Lower entropy factors of sofic systems, Ergodic Theory Dynamical Systems 4 (1984), 541-557.

[B2] _ Shift equivalence and the Jordan form away from zero, Ergodic Theory Dynamical Systems 4 (1984), 367-379.

[BK] M. Boyle and W. Krieger, Periodic points and automorphisms of the shift, Trans. Amer. Math. Soc. 302 (1987), 125-149.

[BMT] M. Boyle, B. Marcus, and P. Trow, Resolving maps and the dimension group for shifts of finite type, Mem. of Amer. Math. Soc. (to appear).

[Br] A. Brumer, On the units of algebraic number fields, Mathematika 14 (1967), 121-124.

[C] E. Coven, Endomorphisms of substitutions minimal sets, Z. Wahrsch. Verw. Gebiete. 20 (1971), 129-133.

[CK] E. Coven and M. Keane, The structure of substitution minimal sets, Trans. Amer. Math. Soc. 162 (1971), 89-102.

[CP] E. Coven and M. Paul, Endomorphisms of irreducible subshifts of finite type, Math. Systems Theory 8 (1974), 167-175.

[DGS] M. Denker, C. Grillenberger, and K. Sigmund, Ergodic theory on compact spaces, Lecture Notes in Math., vol. 527, Springer, New York, 1976.

[E] G. A. Elliott, On the classification of inductive limits of sequences of semisimple finite-dimensional algebras, J. Algebra 38 (1976), 29-44.

[G] E. Gilbert, Synchronization of binary messages, IRE Trans. Inform. Theory 6 (1960), 470-477.

[H] G. Hedlund, Endomorphisms and automorphisms of the shift dynamical system, Math. Systems Theory 3 (1969), 320-375.

[Ka] I. Kaplansky, Infinite Abelian groups, University of Michigan Press, Ann Arbor, 1954.

[Ko] N. Koblitz, p-adic numbers, p-adic analysis, and zeta-functions, Springer, New York, 1977.

[Kr1] W. Krieger, On the subsystems of topological Markov chains, Ergodic Theory Dynamical Systems 2 (1982), 195-202.

[Kr2] _ On dimension functions and topological Markov chains, Invent. Math. 56 (1980), 239250.

[L1] D. Lind, The entropies of topological Markov shifts and a related class of algebraic integers, Ergodic Theory Dynamical Systems 4 (1984), 283-300. 
[L2] _ Entropies of automorphisms of a topological Markov shift, Proc. Amer. Math. Soc. (to appear).

[LS] R. C. Lyndon and P. E. Schupp, Combinatorial group theory, Ergebnisse der Math. 89, Springer, Heidelberg, 1977.

[MKS] W. Magnus, A. Karrass, and D. Solitar, Combinatorial group theory, Dover, New York, 1976.

[N] M. Nasu, Topological conjugacy for sofic systems and extensions of automorphisms of finite subsystems of topological Markov shifts, Proc. Univ. Maryland Special Year in Dynamical Systems 1986-87, Springer (to appear).

[PT] W. Parry and S. Tuncel, Classification problems in ergodic theory, London Math. Soc. Lecture Notes 67, Cambridge University Press, Cambridge, 1982.

[Ro] J. Rotman, The theory of groups, Allyn and Bacon, Boston, Mass., 1973.

[Ry1] J. Ryan, The shift and commutativity, Math. Systems Theory 6 (1972), 82-85.

[Ry2] _ , The shift and commutativity II, Math. Systems Theory 8 (1974), 249-250.

[Se] M. Sears, The automorphisms of the shift dynamical system are relatively sparse, Math. Systems Theory 5 (1971), 228-231.

[Sh] P. Shields, The theory of Bernoulli shifts, Univ. of Chicago Press, Chicago, 1973.

[Sm] S. Smale, Differentiable dynamical systems, Bull. Amer. Math. Soc. 73 (1967), 747-817.

[T] O. Taussky, On a theorem of Latimer and MacDuffee, Canad. J. Math. 1 (1949), 300-302.

[Wa1] J. Wagoner, Realizing symmetries of a subshift of finite type by homeomorphisms of spheres, Bull. Amer. Math. Soc. 14 (1986), 301-03.

[Wa2] J. Wagoner, Markov partitions and $K_{2}$, preprint, Univ. of California, Berkeley, 1985.

[W] P. Walters, An introduction to ergodic theory, Springer, New York, 1982.

[We] E. Weiss, Algebraic number theory, McGraw-Hill, New York, 1963.

[Wi] R. Williams, Classification of subshifts of finite type, Ann. of Math. 98 (1973), 120-153; Errata 99 (1974) 380-381.

Department of Mathematics, University of Maryland, College PaRk, MaryLAND 20742 (Current address of Mike Boyle and Daniel Rudolph)

Department of Mathematics, University of Washington, Seattle, WashingTON 98195 (Current address of Douglas Lind) 